University of Louisville

ThinkIR: The University of Louisville's Institutional Repository

Electronic Theses and Dissertations

$5-2012$

\title{
Development of a hydrogel/polypyrrole interpenetrating network for a potential application as an artificial cardiac muscle.
}

James J. Lee 1978-

University of Louisville

Follow this and additional works at: https://ir.library.louisville.edu/etd

\section{Recommended Citation}

Lee, James J. 1978-, "Development of a hydrogel/polypyrrole interpenetrating network for a potential application as an artificial cardiac muscle." (2012). Electronic Theses and Dissertations. Paper 806. https://doi.org/10.18297/etd/806

This Doctoral Dissertation is brought to you for free and open access by ThinkIR: The University of Louisville's Institutional Repository. It has been accepted for inclusion in Electronic Theses and Dissertations by an authorized administrator of ThinkIR: The University of Louisville's Institutional Repository. This title appears here courtesy of the author, who has retained all other copyrights. For more information, please contact thinkir@louisville.edu. 


\title{
DEVELOPMENT OF A HYDROGEL/POLYPYRROLE INTERPENETRATING NETWORK FOR A POTENTIAL APPLICATION AS AN ARTIFICIAL CARDIAC MUSCLE
}

\author{
By \\ James J Lee \\ B.S., Chemical Engineering, 2001 \\ Virginia Polytechnic Institute and State University
}

\author{
A Dissertation \\ Submitted to the Faculty of the \\ J. B. Speed School of Engineering of the University of Louisville \\ in Partial Fulfillment of the Requirements \\ for the Degree of \\ Doctor of Philosophy \\ Department of Chemical Engineering \\ University of Louisville \\ Louisville, Kentucky
}

May 2012 
Copyright 2012 by James J Lee

All rights reserved 


\section{DEVELOPMENT OF A HYDROGEL/POLYPYRROLE INTERPENETRATING NETWORK \\ FOR A POTENTIAL APPLICATION AS AN ARTIFICIAL CARDIAC MUSCLE}

By

James J Lee

B.S., Chemical Engineering, 2001

Virginia Polytechnic Institute and State University

A Dissertation Approved on

December 7, 2011

By the following Dissertation Committee:

Dr. Gerold A. Willing (Advisor)

Dr. James C. Watters

Dr. Xiao-An Fu

Dr. Andrea S. Gobin 


\title{
DEDICATION
}

\author{
I dedicate this dissertation to
}

\section{My parents}

Mr. Kyung Hyun Lee and Mrs. Yon Hui Lee

for providing me the opportunities towards an exceptional and invaluable education,

and

My younger brother

Jaeff J. Lee

for being my foremost ally in my life pursuits and my greatest friend.

My accomplishments and successes thus far in life are rooted in all of your enduring sacrifice, encouragement, and support. 


\section{ACKNOWLEDGEMENTS}

In acknowledgement of all those who have contributed, encouraged, and helped in putting me in the position to complete this dissertation.

To my major advisor, Dr. Gerold A. Willing, I would like to give my utmost gratitude for his encouragement, guidance, and support. From the inception through the evolution of this project, your encouragement to explore and discover has created a research environment that was challenging and rewarding. Thank you for allowing me to make mistakes and learn from them.

To my dissertation committee, Dr. James C. Watters, Dr. Xiao-An Fu, and Dr. Andrea S. Gobin, I would also like to thank you for your guidance, time, and patience for reviewing and critiquing this body of work.

To Dr. Zach Hilt and Hariharasudhan CD at the University of Kentucky, I am very grateful for your time and guidance in the very early stages of this project. Thank you for providing me the reassurance of having resources available to fall back on during the growing pains of this project.

In the Chemistry Department, thank you Dr. Chris Burns for taking the time to discuss methods and techniques in the organic synthesis of polymer derivatives. From this department I also extend a thank you to Dr. Neal Stolowich for your training with the 
NMR and guidance with troubleshooting issues on the instrument, and Cesar Antonio Masitas, my colleague, for helping schedule and interpret the NMR spectrogram.

In the Conn Center for Renewable Energy Research, thank you to Dr. Jacek Jasinski for taking the time to train me on the SEM, and Rodica McCoy for being available and willing to help with all the odds and ends throughout my graduate career.

I extend my gratitude to my colleague, Jay Stallons, and Dr. McGregor's Lab of the Pharmacology \& Toxicology Department at the Center for Translational Research for providing me the instruction, guidance, support, materials, and supplies to perform cell cultures and biocompatibility assays in your facility.

To my lab mates and colleagues, Sudhira Pasupuletti, Xiaoting Hong, Kan Liu, Jianting Wang, Hanzhu Jin, Yongjie Ren, Andrew Ahn, Bin Hong, Samin Rezania, Chinmay Deshmane, Chandrashekhar Pendyala, Suresh Gubbala, Rajesh Desari, Amber Carrier, Angela Thompson, and Zdravko Salipur, you all have helped me endure during times of distress, provide feedback during times of uncertainty, and share in each other's triumphs. Thank you for your instruction with learning new experimental techniques, support, and encouragement throughout my graduate career.

To Pamela White and Patricia Lumley, a special appreciation and gratitude for your encouragement, help, and support in everything a graduate student could possibly face. Thank you for creating a loving and comfortable department environment.

I am grateful to the NASA Space Grant for Colleges in Kentucky and the Kentucky Space Grant Consortium for awarding me with the Graduate Fellowship and 
providing me the opportunity to initiate much of the preliminary work on this dissertation. I am also grateful to Dr. Ji Su at the Advanced Materials and Processing Branch (AMPB) of the NASA Langley Research Center (LRC) for proving guidance and support with the early developments of this project.

Lastly, I would like to give my gratitude and thanks to my past advisor, Dr. Duane F. Bruley at the University of Maryland Baltimore County for his unwavering support, encouragement, and faith in me. Thank you for your steadfast confidence in my pursuit of my Doctoral degree. 


\section{ABSTRACT \\ DEVELOPMENT OF A HYDROGEL/POLYPYRROLE \\ INTERPENETRATING NETWORK \\ FOR A POTENTIAL APPLICATION \\ AS AN ARTIFICIAL CARDIAC MUSCLE}

James J. Lee

May 2, 2012

Hydrophilic and conductive characteristics in polymers are mutually exclusive of each other. Hydrophilic polymer properties allow absorption of water up to several magnitudes above their dry weight. However, due to their molecular structure, they are not inherently conductive without the aid of a conductive element. Conductive polymers have molecular structures which permit current transfer via a series of delocalized electrons along the polymer backbone. Conversely, due to their organic nature, they are very hydrophobic and will not readily interact with hydrophilic elements.

The motivation of this dissertation was to develop a biocompatible material that could potentially support the muscle like movement and performance of cardiac muscles for the treatment of congestive heart failure. To initiate this investigation, hydrophilic polymers and conductive polymers were separately examined and synthesized. Experimentation led to the selection of methacryamide and pyrrole monomers as the hydrophilic and conductive elements, respectively. Initial efforts to integrate these polymers proved challenging due to the hydrophobic nature of pyrrole and the hydrophilic nature of methacrylamide. To enhance the compatibility of pyrrole within 
the methacrylamide matrix, pyrrole was modified with a hydrophilic group. However, its location on pyrrole had to be selective in order to minimize disruption of the delocalized electrons along the polypyrrole backbone and losses in conductivity.

Synthesis of the polymer compound with the methacryamide hydrogel matrix and modified pyrrole exhibited integration of the pyrrole into the hydrogel by diffusion of the pyrrole monomers into the hydrogel matrix and subsequent polymerization. Copolymers are characterized the combination of monomers in a random or specific arrangement along the backbone of the polymer complex, and composites are regarded as a dispersion of one material in a matrix of the second. This was an integration of two polymers interpenetrating each other into a woven polymer network.

A unique compound was synthesized by integrating two physically incompatible polymer materials. Modification of the pyrrole to a hydrophilic molecule provided the ability to electropolymerize polypyrrole within the hydrogel matrix. An interpenetrating polymer network (IPN) with the integration of both hydrogel and conductive polymer elements were made feasible by this unique piecewise synthesis and design scheme. 


\section{TABLE OF CONTENTS}

\section{PAGE}

DEDICATION

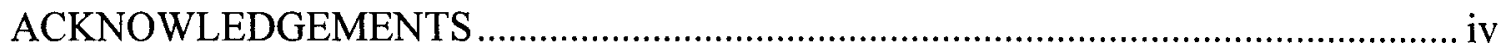

ABSTRACT

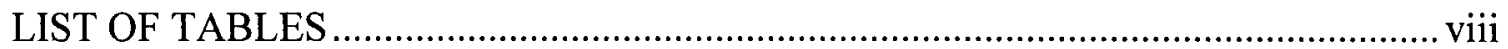

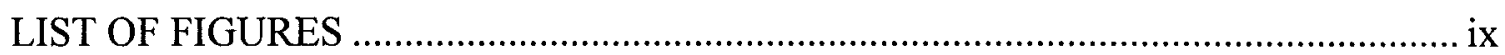

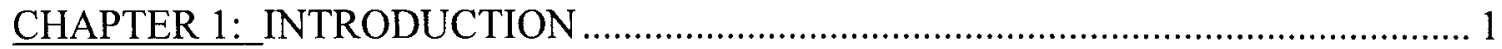

1.1 The Human Heart and Congestive Heart Failure (CHF) ……........................... 1

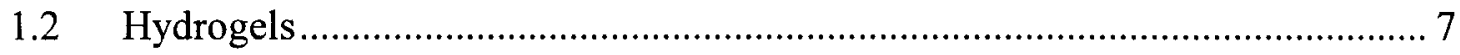

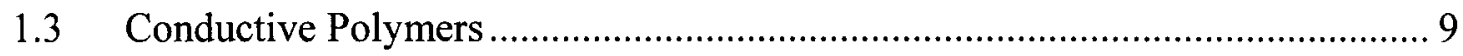

1.4 Polymer Compounds........................................................................... 13

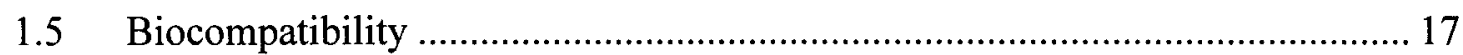

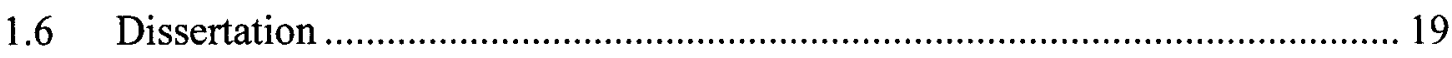

CHAPTER 2:_SYNTHESIS OF THE HYDROGEL COMPONENT …………........... 21

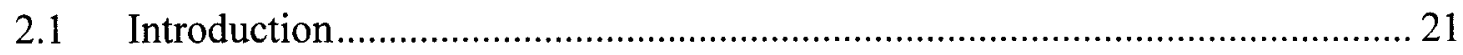

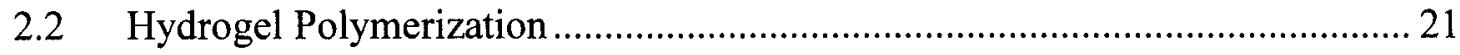

2.2.1 Experimental Procedure - UV polymerization ..................................... 23

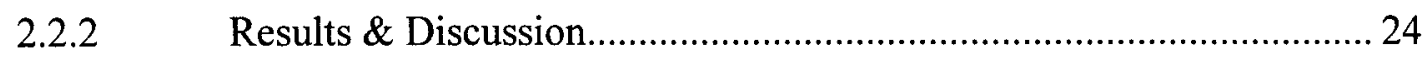

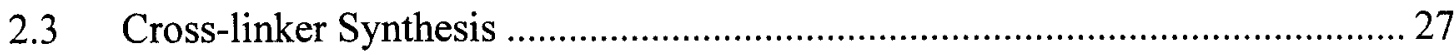


2.3.1 Microwave Assisted Synthesis Reaction for PEGDMA Cross-linker . 28

2.3.2 Experimental Procedure - Microwave Assisted Synthesis Reaction.... 29

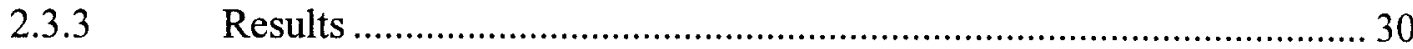

2.4 Hydrogel Cross-linker Polymerization ............................................................. 32

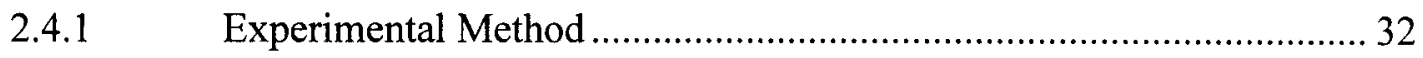

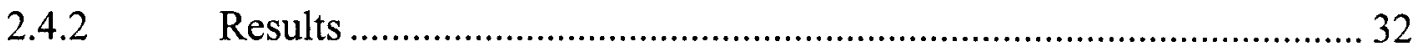

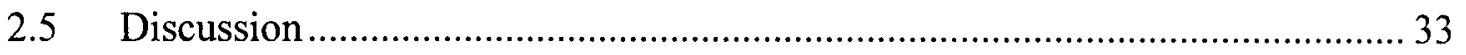

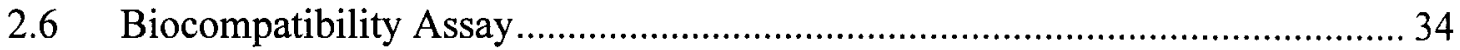

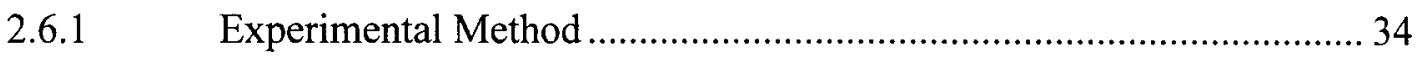

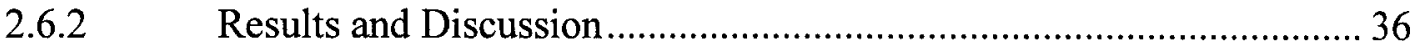

2.7 Deductions on the Synthesis of the Hydrogel................................................. 38

CHAPTER 3: CONDUCTION ENHANCEMENT WITH GOLD NANOPARTICLES39

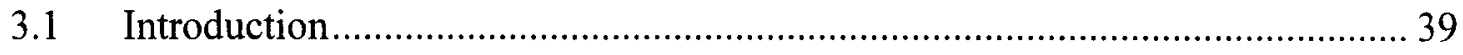

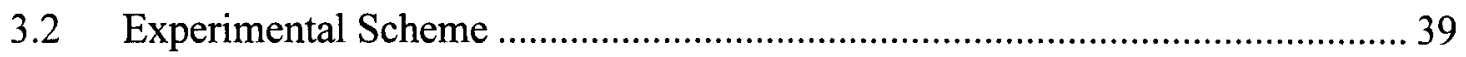

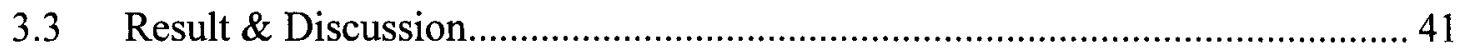

CHAPTER 4: SYNTHESIS OF ELECTROACTIVE COMPONENT …………….......... 45

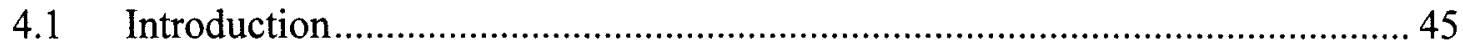

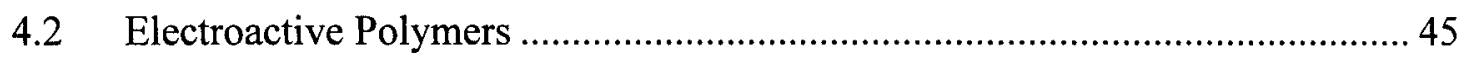

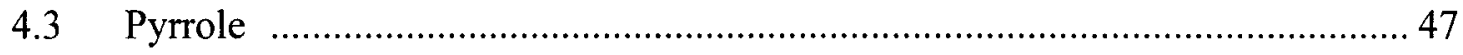

4.4 Electrochemical Polymerization .............................................................. 49

4.4.1 Experimental Method - Electrochemical Polymerization ..................... 50

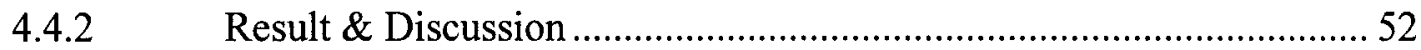


CHAPTER 5: DESIGN AND COMPOSITION OF THE POLYMER COMPOUND... 55

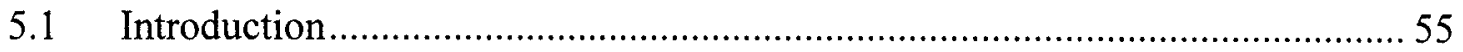

5.2 Design Approach of the Polymer Compound ............................................... 56

5.3 Initial Compound Attempt Experimental Procedure …….................................. 56

5.3.1 Experimental Procedure ……………............................................. 57

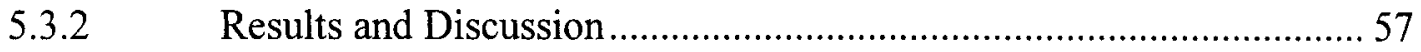

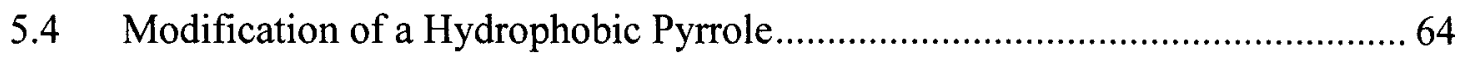

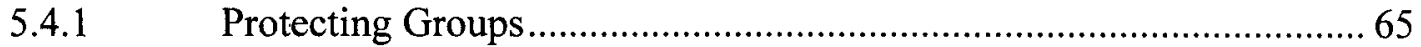

5.4.2 Modification of Pyrrole Monomer .................................................... 69

5.4.3 Modification of the Pyrrole Monomer with a Carboxylic Ion ............. 77

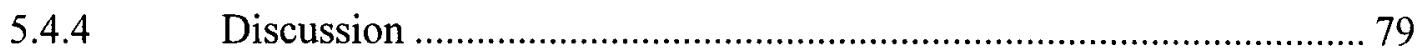

5.5 Polymer Compound Formation with Hydrophilic Pyrrole ............................... 80

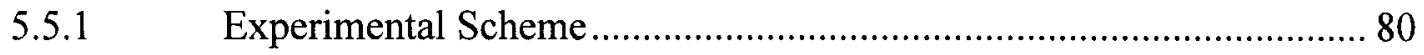

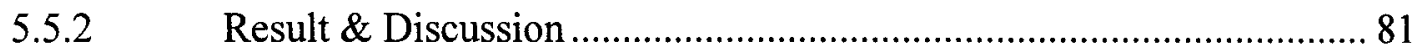

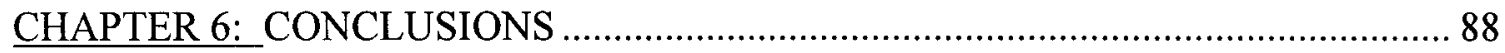

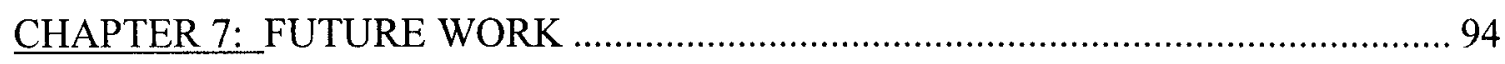

7.1 Hydrogel Characterization and Improvements ................................................ 94

7.2 Improve the Yield on the Synthesis of Hydrophilic Pyrrole Salt ..................... 95

7.3 Increase Polypyrrole Concentration in the Hydrogel........................................ 95

7.4 Enhancement of Conductivity by Gold Nanoparticles ................................... 96

7.5 Effects of the Electrolytic Solution................................................................. 97

7.6 Alternative Methods to Reduce Organics ....................................................... 97

7.7 Identify the Influences of Actuation by Electrical Stimulation ........................ 98 


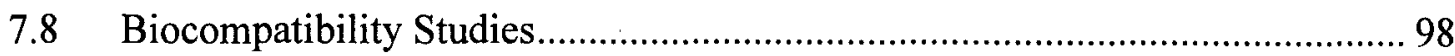

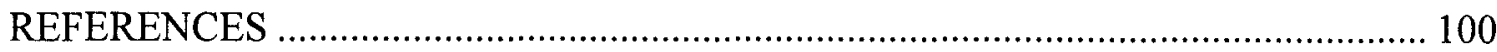

APPENDICES ........................................................................................ 111

Appendix A. Illustration of Photoinitiator Free Radical Generation....................... 111

Appendix B. Protocol for the Bioactivity Assay. ............................................ 112

Appendix B1. L929 Cell Growth and Preparation on 96-Well Plates.................. 112

Appendix B2. Protocol for the MTT Assay ........................................... 113

Appendix C: Table of Literature Citations for the Setup and Conditions for the Electropolymerization of Polypyrrole ...................................... 115

Appendix D. Friedel-Crafts Alkylation Mechanism.......................................... 116

Appendix E. Clemmensen Reduction Mechanism ...................................... 117

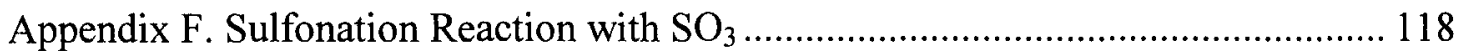

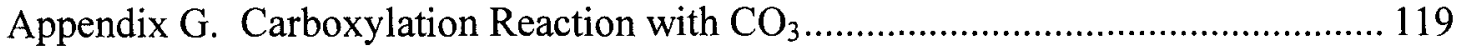

Appendix H. Deprotection Mechanism ........................................................... 120

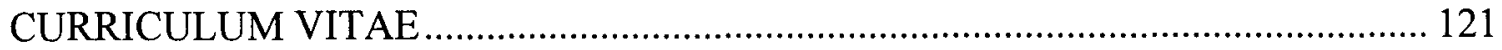




\section{LIST OF TABLES}

TABLE

PAGE

Table 1 Timing of the cardiac cycle for the atrial and ventricular heart chambers. .......... 2

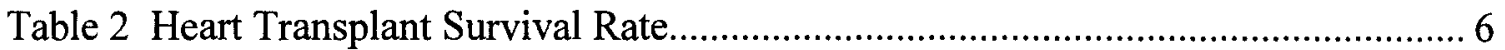

Table 3 Dipole and Dielectric Properties of Some Solvents........................................... 26 


\section{LIST OF FIGURES}

FIGURE

PAGE

Figure 1. Heart Anatomy and Physiology of Congestive Heart Failure (CHF)................. 1

Figure 2. Illustration of Cardiomyoplasty Procedure. ……….......................................... 4

Figure 3. Illustration of Left Ventricular Reduction.................................................. 5

Figure 4. Illustration of Left Ventricular Assist Device (LVAD). ................................... 5

Figure 5. Illustration of the Swelling and Cross-links of Hydrogels................................ 8

Figure 6. Illustration of various conductive polymers............................................... 10

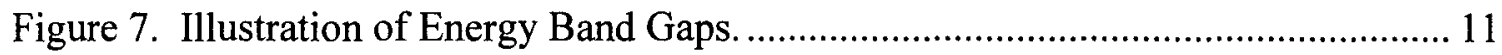

Figure 8. Electroactive polymer (EAP) gripping device. ............................................ 13

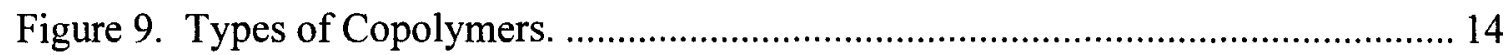

Figure 10. Interpenetrating Polymer Network (IPN) by sequential synthesis................ 16

Figure 11. Illustration of MTT reduction by mitochondria in a viable cell.................... 18

Figure 12. Molecular Structures of Several Hydrogels. ................................................. 22

Figure 13. UV-Photo Initiator 2,2-Dimethoxy-2-phenylacetophenone (DMPA),.......... 22

Figure 14. From left to right; AC, MA, and AA homopolymers................................... 25

Figure 15. Polymerization of Acrylamide and Methacrylamide in Ethanol.................... 25

Figure 16. Polyethyene Glycol (PEG) Dimethacrylate Cross-linker............................... 28

Figure 17. ${ }^{1} \mathrm{H}$ NMR peak correlation to PEGDMA Molecule........................................ 30

Figure 18. ${ }^{1} \mathrm{H}$ NMR of 200 Molecular Weight PEGDMA........................................... 31

Figure 19. ${ }^{1} \mathrm{H}$ NMR of 400 Molecular Weight PEGDMA.............................................. 31 
Figure 20. Preliminary results of cell viability from MTT assay of hydrogel samples... 36

Figure 21. Image of a positive cytotoxic reaction on cell viability following treatment. 37

Figure 22. AFM Image of Au Nanoparticles............................................................ 40

Figure 23. Poly(acrylamide); Au doped (left) and undoped (right)................................ 41

Figure 24. SEM images of GNPs dispersed in poly(acrylamide) at $500 \mathrm{~nm}$ scale......... 42

Figure 25. SEM images of GNPs dispersed in poly(acrylamide) at $200 \mathrm{~nm}$ scale......... 43

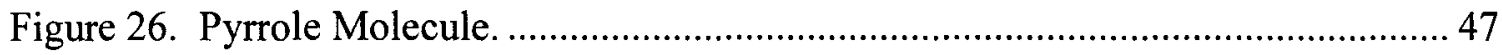

Figure 27. sp3 (left) and sp2 (right) hybridization of pyrrole....................................... 48

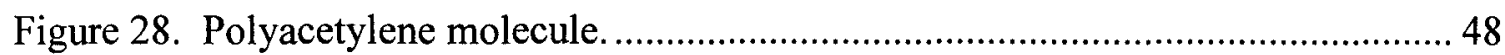

Figure 29. Polypyrrole repeating unit in polymer chain................................................. 49

Figure 30. Illustration of electrochemical cell ......................................................... 51

Figure 31. Photos of Polypyrrole electropolymerized on ITO substrate ........................ 53

Figure 32. FTIR spectra of electropolymerized PPY in mineral oil mull.......................53

Figure 33. FTIR spectra of a) PMA, b) PEGDMA, and c) Mineral Oil.......................... 59

Figure 34. FTIR spectra of electropolymerized PPy .................................................... 60

Figure 35. FTIR spectra, electropolymerization of PPy within PMA on ITO substrate. 60 Figure 36. Molecular Structure of 3-Aminopropyltrioxysilane (APTES)........................ 63

Figure 37. Illustration, silation of substrate surface with (APTES) ..................................63

Figure 38. Illustration of the optical contact angle measurement on a liquid droplet. .... 64

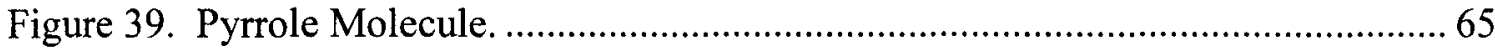

Figure 40. Mechanism of Benzenesulfonyl Protection of Pyrrole monomer. .................. 67

Figure 41. Illustration, protected pyrrole molecule, 1-(Phenylsuflonyl) molecule.......... 68

Figure 42. ${ }^{1} \mathrm{H}$ NMR $\left(\mathrm{CDCl}_{3}\right)$ spectra of 1-(Phenylsulfonyl)pyrrole................................. 69 
Figure 43. Alkylation Reaction Mechansim of 1-(Phenylsulfonyl)Pyrrole

Figure 44. Illustration, 1-[1-(Phenylsulfonyl)-3-pyrrolyl]-4-chloro-1-butanone. 71

Figure 45. ${ }^{1} \mathrm{H}$ NMR $\left(\mathrm{CDCl}_{3}\right)$ spectra of 1-[1-(Phenylsulfonyl)-3-pyrrolyl]-4-chloro-1butanone 72

Figure 46. Illustration, 1-[1-(Phenylsulfonyl)-3-pyrrolyl]-4-chlorobutane molecule...... 73

Figure 47. ${ }^{1} \mathrm{H} \mathrm{NMR}\left(\mathrm{CDCl}_{3}\right)$ spectra of 1-[1-(Phenylsulfonyl)-3-pyrrolyl]-4chlorobutane 74

Figure 48. Illustration, 4-(3-Pyrrolyl)butane sulfonic Acid, Sodium Slat molecule. ...... 76 Figure 49. ${ }^{1} \mathrm{H}$ NMR $\left(\mathrm{D}_{2} \mathrm{O}\right)$ spectra of 4-(3-Pyrrolyl)butanesulfonic Acid, Sodium Salt 76 Figure 50. Illustration, 4-(3-Pyrrolyl)butane carboxylic Acid, Sodium Slat molecule ... 78 Figure 51. 'H NMR $\left(\mathrm{D}_{2} \mathrm{O}\right)$ spectra of 4-(3-Pyrrolyl)butanecarboxylic Acid, Sodium Salt. .78

Figure 52. ${ }^{13} \mathrm{C}$ NMR $\left(\mathrm{D}_{2} \mathrm{O}\right)$ spectra of 4-(3-Pyrrolyl)butanecarboxylic Acid, Sodium Salt 79

Figure 53. FTIR spectra of electropolymerized PPy in mineral oil. 82

Figure 54. FTIR spectra, Electropolymeriztion of PPy within PMA on ITO substrate... 82 Figure 55. FTIR spectra of PMA electropolymerization with 4-(3Pyrrolyl)butanesulfonic Acid, Sodium Salt in water. 83

Figure 56. FTIR spectra of PMA electropolymerization with 4-(3Pyrrolyl)butanecarboxylic Acid, Sodium Salt in water. 84

Figure 57. Image of $100 \%$ confluence for cell growth. 113 


\section{CHAPTER I:}

\section{INTRODUCTION}

\subsection{The Human Heart and Congestive Heart Failure (CHF)}

The human heart weighs an average of 300 grams, half of which is cardiac muscle. Divided into four main chambers, the left and right half sides both consist of an atrium that receives blood and a ventricle that pumps the blood away from the heart as illustrated in Figure 1.

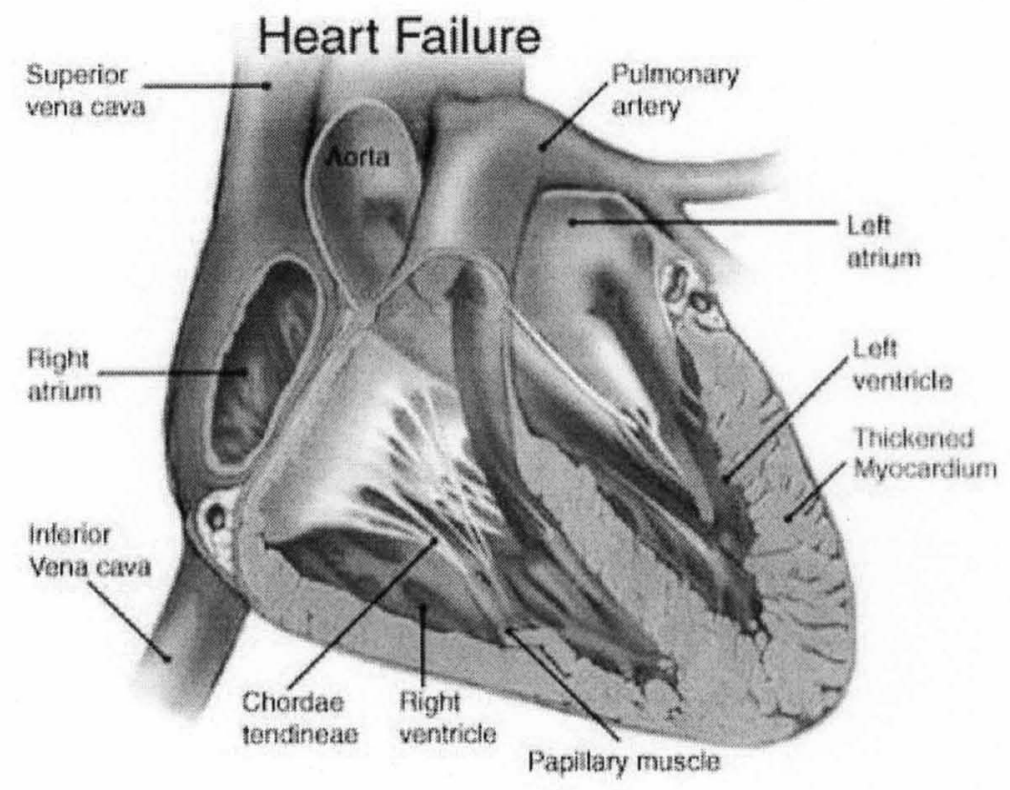

Figure 1. Heart Anatomy and Physiology of Congestive Heart Failure (CHF) [1]. 
The major cardiac muscle providing the pumping action necessary to circulate the blood throughout our body is the left ventricle. As seen in Figure 1 the muscle wall of the left ventricle is very thick as compared to the muscles in the other chambers of the heart. Spontaneous depolarization and conductivity causes the cardiac muscle to contract via electrochemical action potential. This action potential is created by ion channels that regulate the osmotic relationships of $\mathrm{Na}^{+}, \mathrm{K}^{+}$, and $\mathrm{Ca}^{2+}$ between the inside and outside of the muscle cell [2]. The membrane potential of the cardiac muscle cells is between -50 and $-60 \mathrm{mV}$, which indicates that it is electrically negative to the exterior. The velocity of electrical conduction through the atria and ventricular muscles is about $0.3 \mathrm{~m} / \mathrm{sec}$. The cardiac muscle follows the all or none law, so the muscle only contracts if the stimulus is strong enough to cause depolarization. Unlike skeletal muscle the cardiac muscle has a long refractory period, so it cannot be stimulated to contract again until it has a definite period of time has passed to allow the muscle to polarize. The complete cycle of the heart contraction takes about $0.8 \mathrm{sec}$ if the heart is at a normal resting rate of about 70 beats/minute. In a cycle, the heart chambers go through three steps: systole (contraction), diastole (relaxation), and diastasis (rest). The muscles in the atrium are slightly different from those of the ventricles in this cycle as shown in Table 1.

\section{Table 1}

Timing of the cardiac cycle for the atrial and ventricular heart chambers

\begin{tabular}{cccc}
\hline$(\mathrm{sec})$ & Systole & Diastole & Diastasis \\
\hline Atrial & 0.1 & 0.1 & 0.6 \\
Ventricular & 0.2 & 0.2 & 0.4 \\
\hline
\end{tabular}


Congestive heart failure is a condition when the heart cannot maintain the necessary pressure within the cardiovascular system to pump enough blood to supply the body's other organs. Typically this is a progressive deterioration of the heart's ability to contract and eject blood out of the ventricle (systolic dysfunction) [3]. The thickened myocardium also causes the muscles to stiffen causing the inability to fully fill the ventricle (diastolic dysfunction). The 1996 United States statistics reported that heart failure was prevalent in about 4.8 million Americans, which is about $1.8 \%$ of the population or 1 in $56[3,4]$. Every year, heart failure causes about $10 \%$ of deaths in the United State [5], and about 260,000 deaths are related to the condition [6]. It is the $4^{\text {th }}$, $2^{\text {nd }}$ and $1^{\text {st }}$ leading cause of death among those in the age groups $25-44,45-65$, and over 65, respectively [6]. A 2002 report by National Heart, Lung and Blood Institute (NHLBI), estimated a cost of $\$ 23.2$ billion for managing congestive heart failure.

The most general method of managing congestive heart failure (CHF) is pharmacologically. However, pharmacologics only help to relieve the symptoms and delay the progression of CHF, in addition to maintaining the normal fluid level in the circulatory system. There are radical new methods of surgical remodeling to treat CHF. One procedure is called cardiomyoplasty where the latissimus dorsi muscle from the patient's back is lifted and then placed as a graft to be wrapped around the ventricles of the failing heart $[7,8]$ as illustrated in Figure 2 . This muscle is electrically stimulated to contract during ventricular systole. About $80 \%$ of the patients who had cardiomyoplasty indicated improvement in the quality of life months after the operation [9]. The improvements may be related to the effective assistance in the systole of the ventricle 
[10], decease of the lateral wall tension [11], decrease in the dilatation of the ventricular chamber [12], and increase in the blood flow to the ischemic myocardium [13].

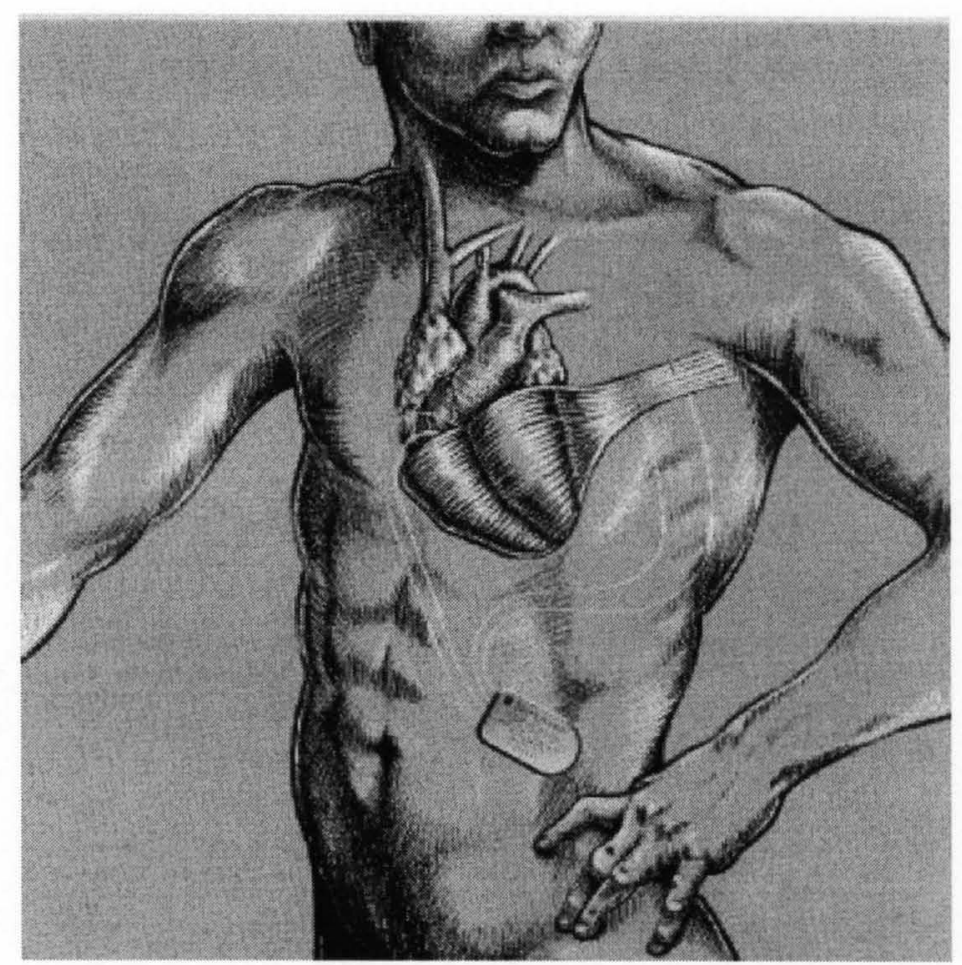

Figure 2. Illustration of Cardiomyoplasty Procedure [14].

In cases where the heart is enlarged and weakened, another surgical procedure invented by Dr. Randas Batista, a Brazilian doctor, in 1994, is a Partial left ventriculectomy or a surgical reduction remodeling of the left ventricular. It is also known as the Batista procedure. Figure 3 illustrates this procedure in which a section of the left ventricular heart muscle is removed and the ventricle size is effectively reduced to its normal size [15]. This allows the ventricle to effectively pump the proper fluid volume and restore pumping pressure and blood flow $[16,17,18]$. The procedure only provided temporary improvement with a significant early failure rate. A study at the 


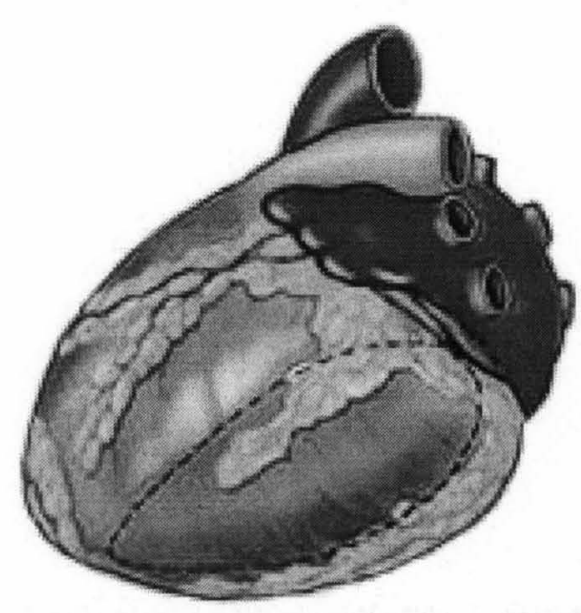

A. Enlarged heart before LVR surgery. Dotted lines indicate slice to be removed by surgeon.

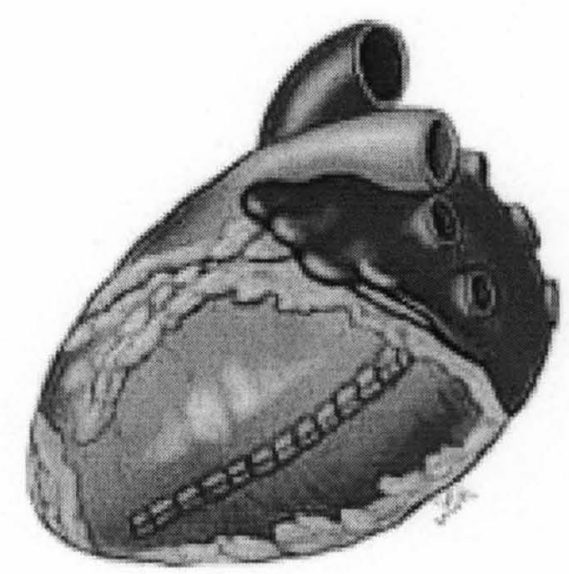

B. Heart after LVR surgery.

Figure 3. Illustration of Left Ventricular Reduction [19].

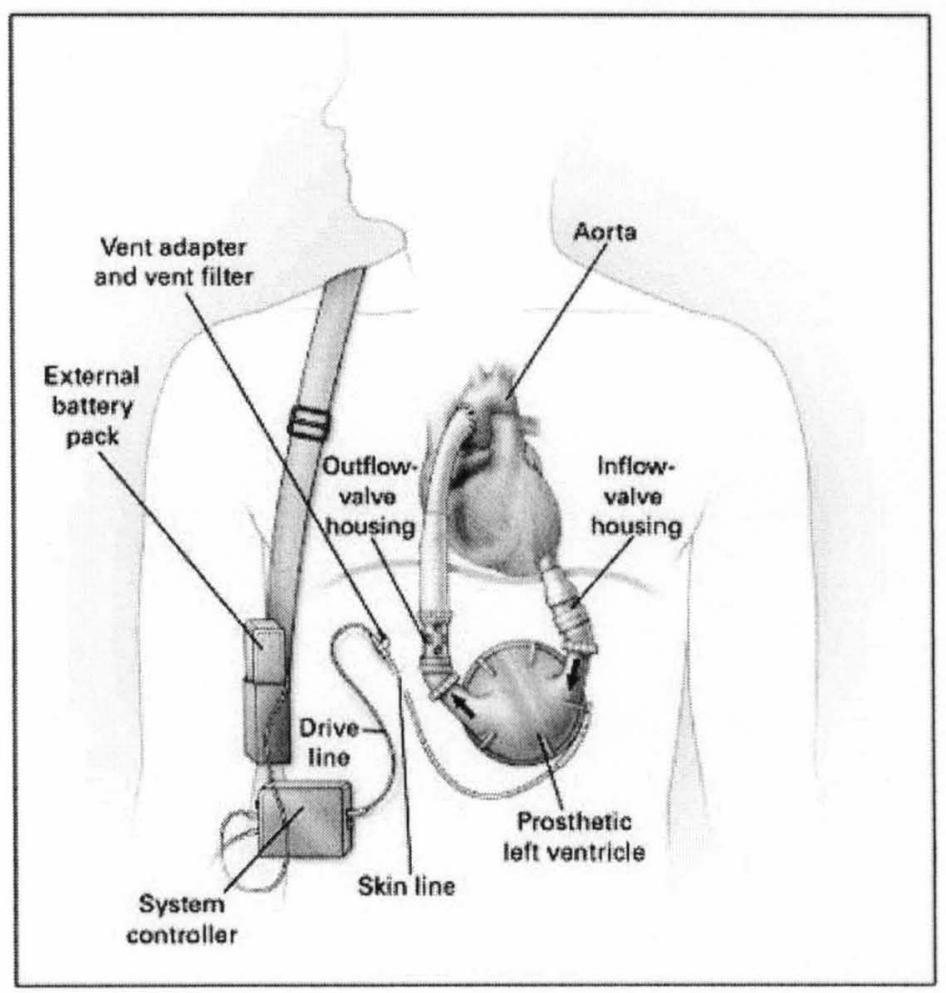

Figure 4. Illustration of Left Ventricular Assist Device (LVAD) [20]. 
Cleveland Clinic showed that after 3 years, of the patients receiving the procedure, $60 \%$ survived the procedure and $26 \%$ were event free of failure [21].There are also devices to assist a patient with CHF whose left ventricular ejection fraction is less than $35 \%$ of normal capacity. A left ventricular assist device (LVAD) is a battery-operated mechanical pump-type device implanted above the abdomen to take blood from the left ventricle and pump it through the aorta [22]. This is illustrated in Figure 4. LVADs are commonly used in patients who are awaiting a transplant, and are only a temporary remedy that is often utilized for a few weeks or months.

When these preceding methods fail in the management of CHF, the ultimate measure for treating CHF is by transplant surgery or implantation of an artificial heart. The American Heart Association reported 2,125 and 2,192 transplants in the year 2005 and 2006 , respectively. The percentage of transplant recipients at ages 35-49 and 50-64 are $20.0 \%$ and $55.3 \%$, respectively [23]. The survival rates among men and women receiving transplants are shown in Table 2 .

\section{Table 2}

Heart Transplant Survival Rate [23]

\begin{tabular}{cccc}
\hline \# of Years & 1 & 2 & 3 \\
\hline Men & $87.4 \%$ & $78.7 \%$ & $72.3 \%$ \\
Women & $85.5 \%$ & $75.9 \%$ & $67.6 \%$ \\
\hline
\end{tabular}


In a comparable approach to the cardiomyoplasty procedure, it could be practical to fashion a biocompatible material that can mimic the application of the latissimus dorsi muscle. If a biocompatible material could be designed and developed to perform equally like muscle, an artificial device in place of the patient's own muscle could assist heart pumping in cardiac patients with congestive heart failure.

\subsection{Hydrogels}

Hydrogels are very effective for use in biomedical applications due to their biocompatibility. Hydrogels are cross-linked polymers that are hydrophilic. The biocompatibility of the hydrogel is primarily the result of the similarity of its water content as compared to that of living tissue which allows them to be permeable to metabolites. Due to the cross-linking within hydrogels, they tend to be inert to biological processes, resist degradation and absorption by the body, will not degrade during heat sterilization within an autoclave, all while maintaining the ability to be molded into various shapes and forms [24]. Common uses of hydrogels in practical applications are in drug delivery, contact lenses, artificial cartilage, and medical aids for wound management.

Hydrogels can be synthesized from homopolymers, copolymers, multipolymers, and interpenetrating polymeric structures where at least one type of monomer is hydrophilic. Cross-linking is accomplished by interconnecting the polymer chains through carbon atoms, chemical bridges, van der Waals forces, hydrogen bonds, and/or molecular entanglements. The advantage of cross-linking is that it inhibits close packing of the polymer chains and the formation of crystalline regions. The chemical cross-links 
formed are mechanically and thermally stable. Multifunctional junctions are the most common types of cross-links. Defects, such as unused functional groups and chain loops, in the cross-linked structure can decrease the overall mechanical strength and physical properties of the hydrogel.
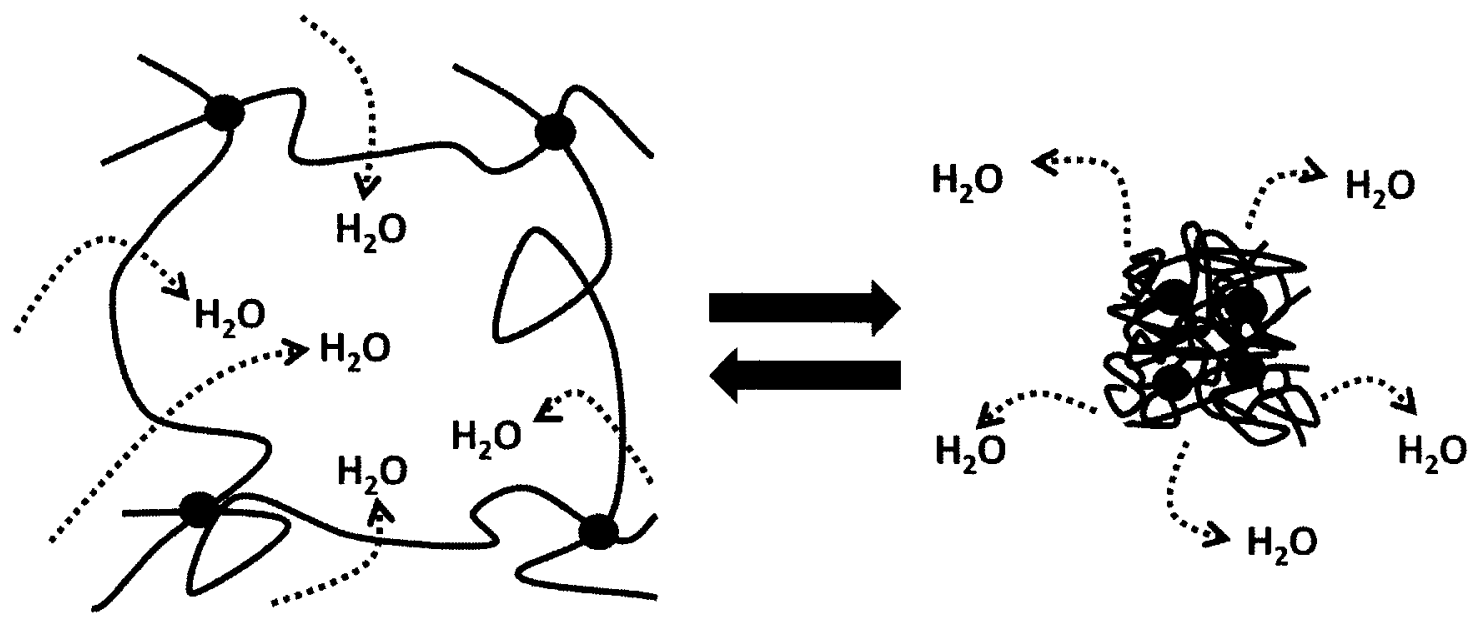

Figure 5. Illustration of the Swelling and Cross-links of Hydrogels.

The force of the swelling of the hydrogel is caused by a variation of the water that is in equilibrium with the networked cross-linked structure as seen in Figure 5. The degree of swelling can affect the mechanical properties of the hydrogel. For example, higher water content within the hydrogel matrix leads to higher rigidity due to expanded cross-links. The swelling properties of the hydrogel can be modified by copolymerizing it with monomers of varying hydrophobicity or cross-linker length.

The diffusion of molecules into and out of the hydrogel provides the mechanism for the polymer to shape-change through the expansion and contraction of the cross- 
linked matrix. The driving force of molecules in and out of the hydrogel is typically environmentally induced as a result of changing $\mathrm{pH}$, temperature, or electrolyte concentration. These shape-changing hydrogels or ionic polymer gels (IPGs) do not require a large voltage and can exhibit large bending displacements, but have a slow responsive change in shape due to the rate of diffusion of molecules, exhibit a relatively low bending actuation force, and require the use of an electrolyte which intern requires their use in an aqueous environment [25]. Hydrogels offer a material with high compatibility in biological systems, but the characteristics of shape-changing with higher actuation forces and faster response will need to be found in another class of material.

\subsection{Conductive Polymers}

Conductive polymers may provide the type of polymeric material that can provide the speed and force necessary for an artificial muscle-like device. Conductive polymers are organic polymers that conduct electricity. Most common polymers such as polyethylene have valence electrons covalently bound in the $\mathrm{sp}^{3}$ hybridized state. These sigma-bound electrons have low mobility and have very little contribution to the electrical conductivity of the polymer. In conducting polymers the backbone is continuously $\mathrm{sp}^{2}$ hybridized throughout the carbon centers. The one valence electron in the $\mathrm{p}_{\mathrm{z}}$ orbital is orthogonal to the other three sigma-bonds of the carbon center. The delocalized electrons are highly mobile within the material when it is "doped" by oxidation, which removes some of these delocalized electrons. The conjugated p-orbitals form a one-dimensional electric band, which become mobile when it is partially emptied. 
Orientation of the polymer chains results in a highly anisotropic material having a maximum conductivity along the direction of orientation [26]. Conductivities of these
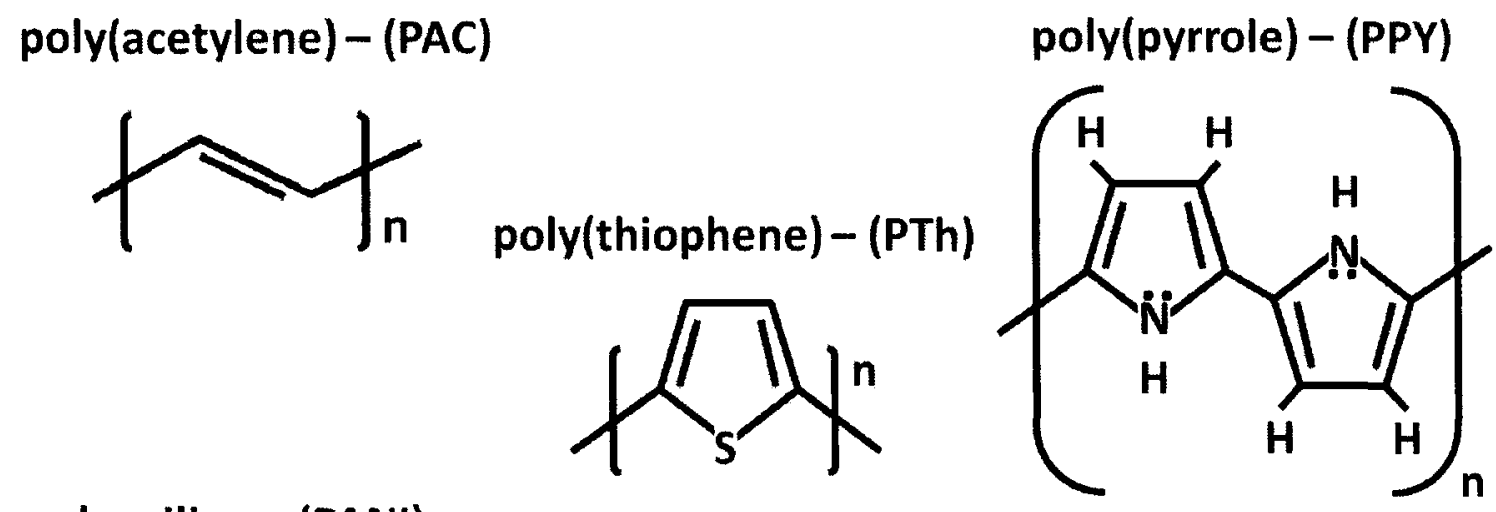

polyaniline - (PANI)
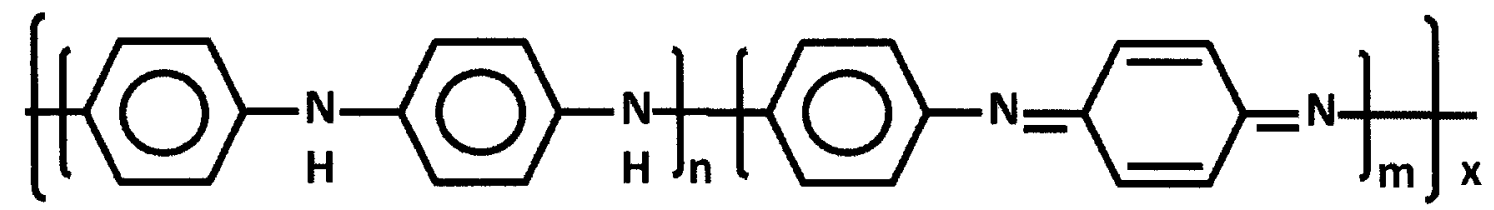

Figure 6. Illustration of various conductive polymers.

polymers have been seen as high as $1.5 \times 10^{7} \mathrm{~S} / \mathrm{m}$, which corresponds to about one fourth of the conductivity of copper $\left(6.0 \times 10^{7} \mathrm{~S} / \mathrm{m}\right)$ by size [26]. Examples of some wellstudied conductive polymers are shown in above in Figure 6.

Much like silicon semiconductors, conductive polymers can be doped by reduction, which is when electrons are added to an unfilled band. However, in practice most organic conductors are doped oxidatively to produce p-type materials. Unlike semiconductors the dopant atoms or molecules do not replace any of the polymer atoms. The dopant atoms appear to lead to the formation of a new energy band that overlaps the valence and conduction bands of the polymer as is illustrated in Figure 7. This provides a 
partially filled band and a high concentration of free electrons or holes. Undoped conductive polymers are at best semiconductors or insulators. The energy gap may be greater than $2 \mathrm{eV}$ in the undoped conductive polymer, and therefore the materials have a low electrical conductivity only between $10^{-8}$ to $10^{-6} \mathrm{~S} / \mathrm{m}$ in their undoped state.

However, the electrical conductivity of conductive polymer are still greater than most insulating polymers which have electrical conductivities ranging between $10^{-17}$ to $10^{-9}$ $\mathrm{S} / \mathrm{m}$. With less than $1 \%$ level of dopant through the electrical conductivity of conductive polymers can be increased by several orders of magnitude. Electrical conductivity values can be increased from between $10^{-8}$ and $10^{-6} \mathrm{~S} / \mathrm{m}$ in their undoped state up to values between $10^{1} \mathrm{~S} / \mathrm{m}$ and $10^{6} \mathrm{~S} / \mathrm{m}$ at a saturated doped state. For example, the highest values reported for the electrical conductivity of polyacetylene have been about $8.0 \times 10^{7} \mathrm{~S} / \mathrm{m}$ [27].

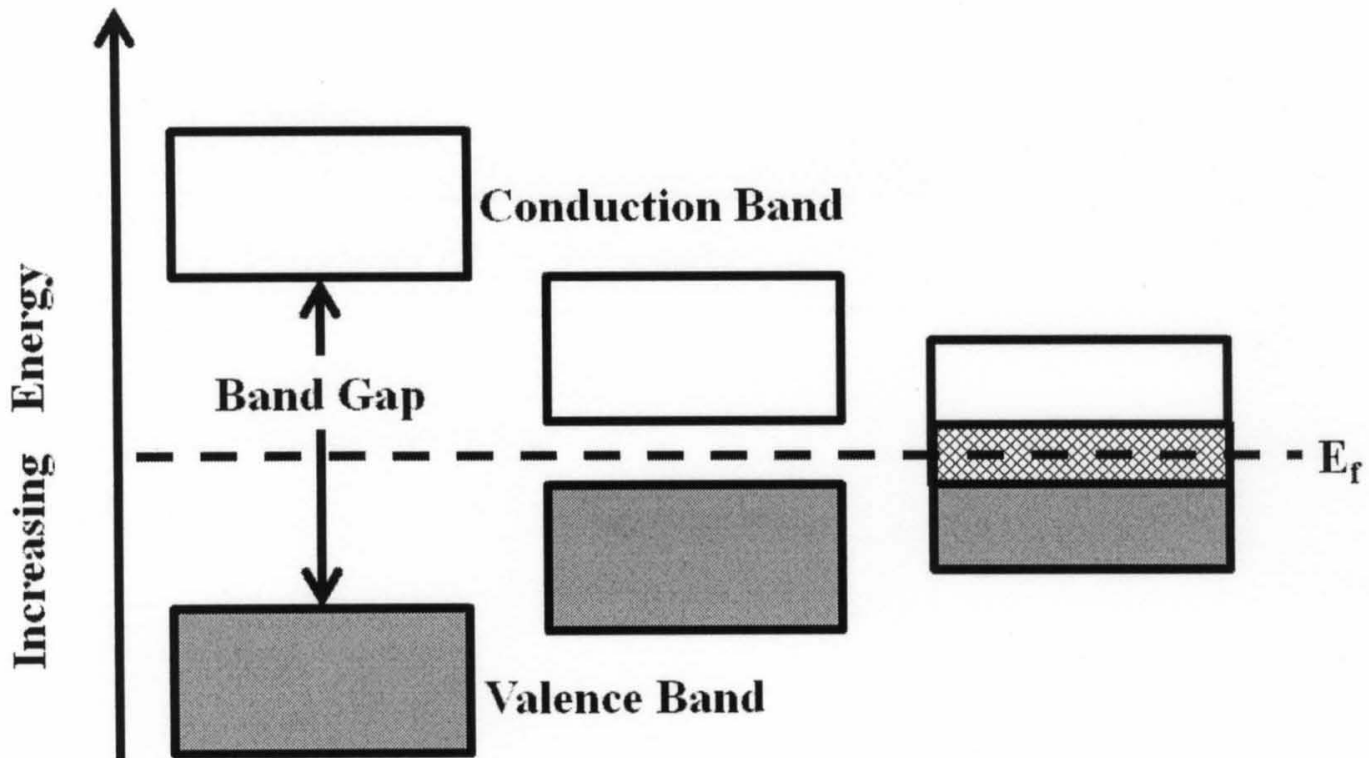
Insulator
Semiconductor
Conductor

Figure 7. Illustration of Energy Band Gaps. 
The facts that these polymers have low densities, are highly flexible, and are easy to produce contribute to their potential application and replacement for inorganic and metallic conductors and semiconductors [26]. Conductive polymers can be utilized in antistatic materials [28],organic light-emitting diodes, batteries, organic solar cells, organic inks for printing electronic circuits, actuators, electrochromic displays [29], supercapacitors, biosenors, electromagnetic organic shielding [, and as replacements for transparent conductors such as indium tin oxide [26]. However, a limitation of conductive polymers is that they currently cannot be easily processed on a large-scale. Unlike most common plastics, they cannot be easily formed or molded by being heated. Conductive polymers are typically polymerized using organic synthesis techniques. Methods of polymerization mainly involve oxidative coupling of the monocyclic precursor such as dehydrogenation. Since conductive polymers require oxidative doping, the polymerization methods in organic solvents or water are crucial to developing the properties of the material. Another major challenge of polymerization involves the low solubility of the polymeric monomer of conductive polymers in aqueous solutions. When doped, such materials are salt-like (polymer-salt) and therefore can have diminished solubility in organic solvents and water. The charged organic backbone is often unstable towards atmospheric moisture. The synthesis of these materials may require multiple steps, or the introduction of substituents to the monomer to solubilize and/or stabilize the polymer, which can further complicate the synthesis. 

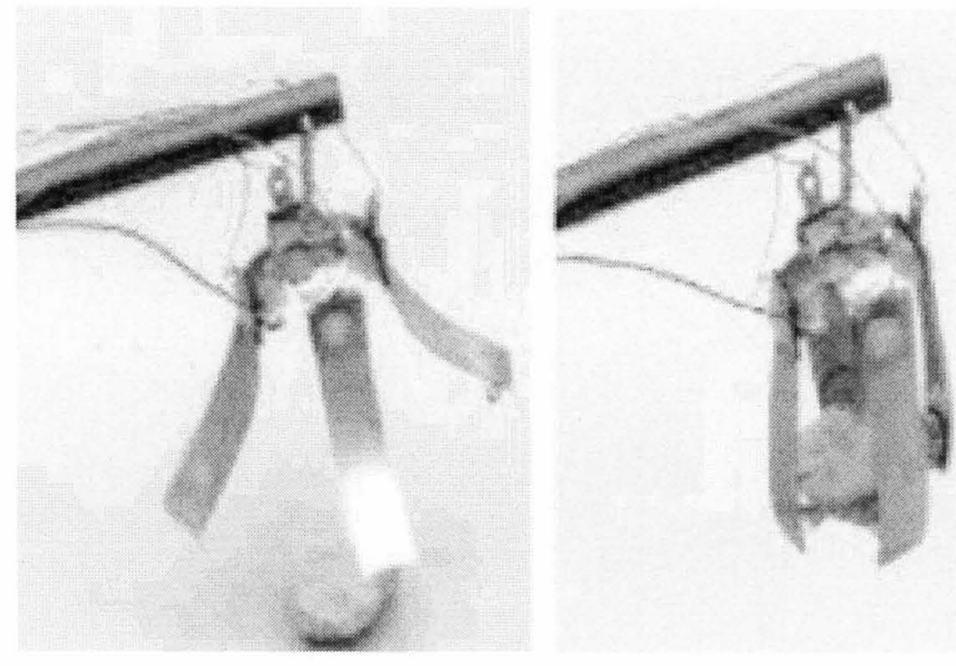

Figure 8. Electroactive polymer (EAP) gripping device [30].

Several conductive polymers can shape-change by electrical stimulation. These electroactive polymers (EAPs) can rapidly actuate with large actuation forces [31]. Figure 8 demonstrates the application of an EAP device. When a voltage is applied to the EAP, the fingers bend in order to surround the rock. Conductive polymers may provide the type of polymeric material that can provide the speed and force necessary for an artificial muscle-like device. However, the integration of the EAP with the hydrogel is difficult because the EAP is organic and inherently hydrophobic. Various schemes for compounding the hydrogel and EAP were examined in this work.

\subsection{Polymer Compounds}

There are several methods of combining the hydrogel and conductive polymer into a polymer compound. Copolymers, composites, and interpenetrating networks are 
various types of polymer compounds that may permit the integration of the hydrogel and EAP with the desired properties inherent in both materials.

Copolymers are polymers with two or more monomeric species. Copolymers are classified by the arrangement of the monomers along the polymer chain. Figure 9 illustrates these copolymers where alternating (1) have units $A$ and $B$ that regularly alternate, periodic (2) have units $A$ and $B$ that repeat in specific sequence, statistical or random (3) have units $A$ or $B$ arranged by a numerical quantity that can be calculated by mole fraction of the monomer, and block (4) have two or more homopolymer subunits covalently bonded to each other. Copolymers structured in a single chain may be classified as linear or branched if one or more polymer side chains are structured off a single main chain. Graft copolymers (5) are branched copolymers composed of structurally distinctive homopolymer or copolymer side chains grafted to the main chain.

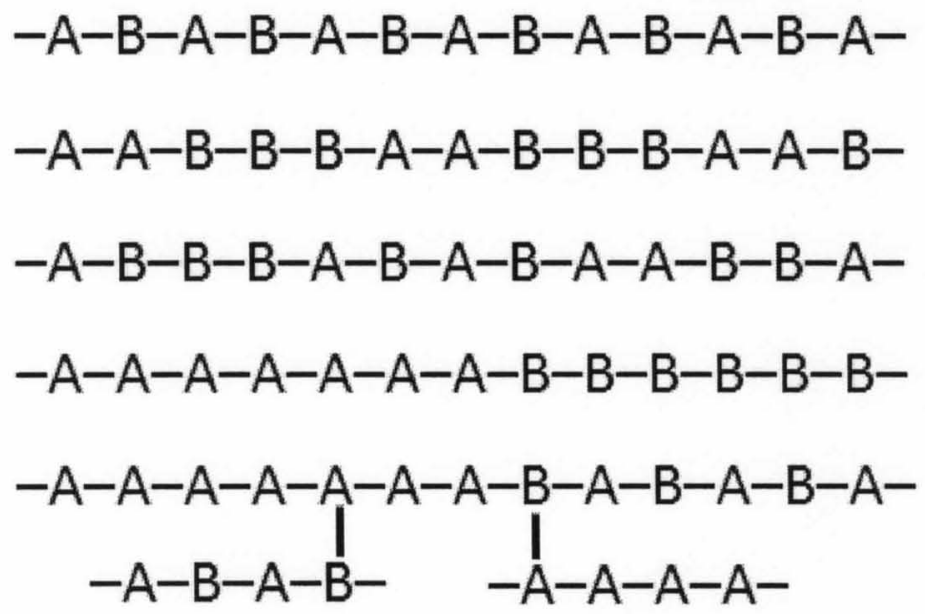

Figure 9. Types of Copolymers. 
Composite materials are engineered or naturally occurring materials that are composed of two or more materials with significantly different physical or chemical properties. These materials remain separate and distinct at the macroscopic or microscopic scale within the finished structure. The physical properties of composite materials are generally anisotropic (different) depending on the direction of the applied force or load. For example the composite may be more rigid when force is applied to the material in one direction verses another. The constituent materials are labeled as either matrices (continuous phase) or dispersed. The matrix material surrounds and supports the dispersed materials by maintaining a structural framework. The purpose of the matrix is also to protect the dispersed material from damage, failure, or reaction with the environment. The dispersed material imparts its special mechanical or physical properties to enhance the matrix properties. The variety of matrix and dispersed materials allows the designer to optimize the combination to achieve the properties desired for a specific application [26]. The bond between the matrix and dispersed phases should be adequate enough to minimize separation or pull-out of the dispersed element from the matrix.

Interpenetrating polymer networks (IPN) are polymer networks comprised of two or more polymers that is interlaced on a molecular scale, but is not covalently bonded to each other. More importantly, the polymers cannot be separated unless chemical bonds are broken [32]. Similar to block copolymers, the polymer domain of the IPN is determined by the cross-link length and density. IPNs may be prepared by either sequential or simultaneous methods. The sequential method utilizes polymerization of the each individual polymer component following the completion of the previous 
component in the network as illustrated in Figure 10. The simultaneous method utilizes the technique by which all components are polymerized concurrently. Semiinterpenetrating networks (SIPN) are IPNs with one or more of the secondary polymer not fully cross-linked throughout the primary polymer matrix. The secondary polymer(s) penetrates the primary network by a series of linear or branched polymer(s) at the molecular scale as illustrated in Figure 10. It may be possible for the linear or branched polymer to be incorporated into the network by means other than polymerization such as swelling and diffusion into the network [33]. The main characteristic difference of SIPNs to IPNs is that the secondary polymer network may be separated from the constituent network without breaking chemical bonds. SIPNs are more characteristic of most polymer blends.

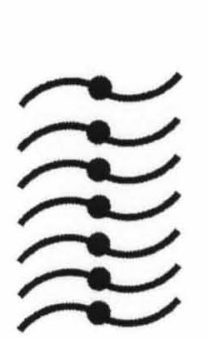

Monomer A

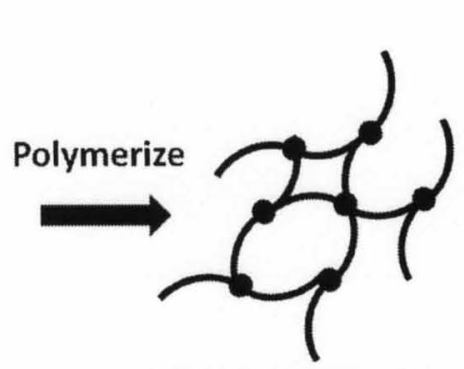

Polymer Network A

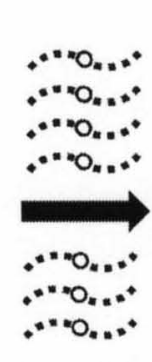

Monomer B

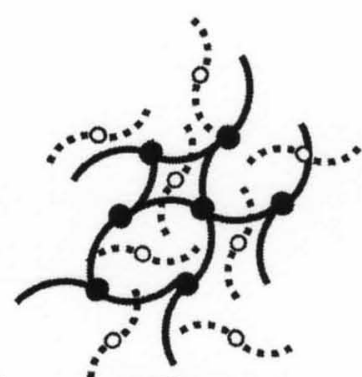

Diffuse

Monomer B
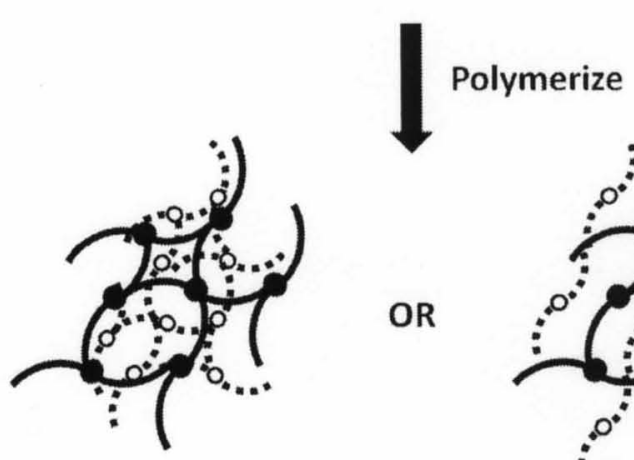

Integrated Polymer Network (IPN)

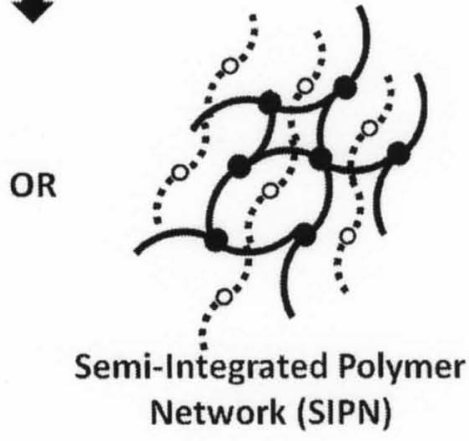

Figure 10. Interpenetrating Polymer Network (IPN) by sequential synthesis. 
There are several approaches to integrating the hydrogel and conductive polymer. One option is that the hydrogel and conductive polymer elements may be combined as a copolymer. However, as a copolymer, the compound loses the continuous $\mathrm{sp}^{2}$ hybridized backbone throughout the carbon centered chain and ultimately the conductivity of the material. However, as a composite, if the dispersion of the conductive polymer is not at a concentration to permit conduction throughout the hydrogel matrix, the compound may not be conductive. Also, if the compound is designed with sandwiched layers of hydrogel and conductive polymers, the composite may separate because the conductive polymer is hydrophobic which creates a chemical mismatch between the layers. SIPNs can potentially provide the most effective anisotropic compound with the conductive polymer interpenetrating a hydrogel matrix in a design that promotes conduction in a specific direction.

\subsection{Biocompatibility}

To demonstrate biocompatibility, the important material characteristics are a degree of inertness to biological processes, resistance to degradation, permeability to metabolites, resistance to absorption by the body, and stability during autoclaving. It is preferable for the polymerization reaction to occur in an aqueous system. Many of the chosen monomers for both hydrogels and conductive polymers are very toxic and poisonous (i.e. neurotoxin), but are very stable and inert once polymerized. International Organization of Standardization (ISO) for biocompatibility outlined in ISO 10993 and FDA G95-1 Guidelines lists an assay using L929 mouse fibroblast cells to determine cytotoxicity on materials to be implanted in vivo [34,35]. Cytotoxicity testing is a rapid, 
standardized, sensitive, and inexpensive means to determine whether a material contains significant quantities of biologically harmful extractables. The toxicity of the polymer may be measured by a MTT assay for metabolic cell activity of a mouse fibroblast cell line as demonstrated by Meulen et al. [36]. The MTT assay can rapidly determine biological reactivity of the cells to a test material by colormetrically quantifying metabolic activity at $560 \mathrm{~nm}$. Figure 11 shows the reduction reaction of the tetrazolium (WST-8, yellow) to formazan (orange dye) when it enters the cell and is reduced by the mitochondria inside viable cells. This assay determines biological reactivity of the cells to a test material. The cells are allowed to grow to confluency in a plate. The prepared test material is transferred onto the cell layer for at least 24 hours at $37^{\circ} \mathrm{C}$ and both positive and negative controls are tested similarly. The degree of cytotoxicity is graded on a scale from no reactivity to severe reactivity. This is a good general indication of basic cellular responses to the test material.

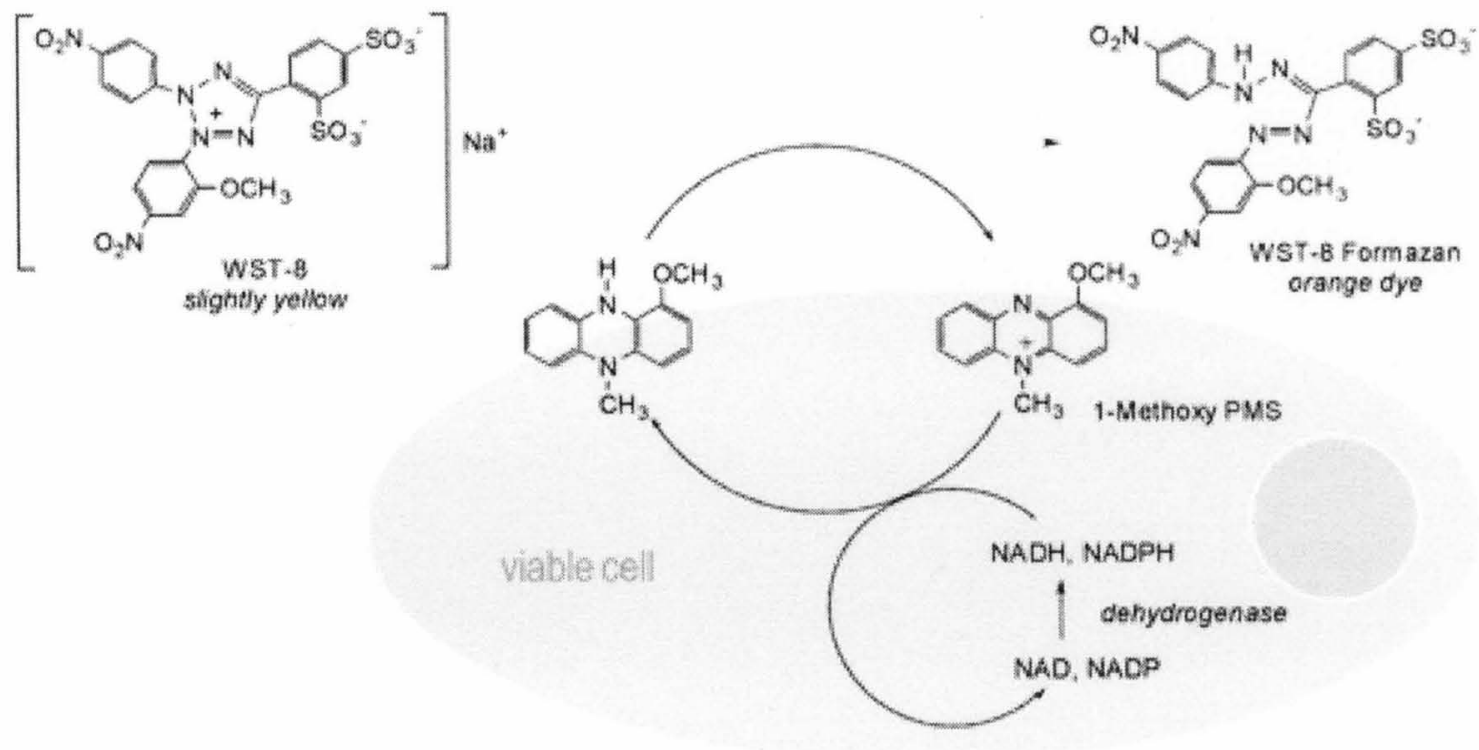

Figure 11. Illustration of MTT reduction by mitochondria in a viable cell. 
Among most polymers, hydrogels are very effective for biomedical applications due to their biocompatibility. Its effective biomedical application is attributed to these following characteristics of biocompatibility: its similarity of the water content to living tissue, being inert to biological processes, resistance to degradation, permeability to metabolites, resistance to absorption by the body, thermal stability to heat sterilization, and the ability to mold into various shapes and forms [37].

With regards to biocompatibility, a potential long-term biocompatibility concern with the polymer implants arose from an event with a hydrogel lens. The FDA performed a five year follow-up study of potential side-effects of the first implantable hydrogel lens. Tests showed a steady loss of endothelial cells at $1.8 \%$ per year. It may be possible that if this device were implanted in the body for a long term, it may cause the surrounding tissue to deteriorate. Negatively charged sulfonate groups in the polymer composite may reduce protein adsorption or platelet adhesion as demonstrated by some researchers [38]. It is imperative that attention be given towards the polymer so that its chemistry is stable and does not have reactive groups that could cause the material to generate an immune response for long term application.

\subsection{Dissertation}

This dissertation investigates the synthesis of a novel polymer compound that integrates properties that are both conductive and hydrophilic for the potential application for a device treating congestive heart failure. Conductivity in hydrogels and hydrophilicity in conductive polymers are physical properties limited to the materials themselves unless they are modified with elements that can provide those physical traits. 
The design and synthesis of this polymer compound was developed piecewise in stages. It was important to consider polymerization techniques that minimize the use of organics and promote polymerization of biocompatible product. The first step in our study was to experiment with the hydrogels and evaluate how it may contribute toward the hydrophilic component of the compound. Photopolymerization with ultraviolet (UV) light was utilized as the polymerization method for the hydrogel. The hydrogel was cross-linked with the appropriate proportion and molecular weight of a cross-linker to enhance structural stability and swelling properties of the hydrogel. Secondly, electroactive polymers were examined as the contributing component to the conductive aspect of the polymer composite. Electropolymerization was the method of polymer synthesis chosen to minimize the utilization of organic chemicals. Pyrrole was the electroactive monomer selected for the electropolymerization study because it is the most extensively utilized monomer for electroactive polymer synthesis. The electrochemical cell was designed using glass coated with indium tin oxide, which is significantly less expensive than platinum foil. Lastly, to synthesize the shape-changing conductive hydrogel polymer compound, three material elements were integrating in a piecewise design scheme utilizing multiple synthesis and polymerization techniques. The hydrogel, cross-linker, and pyrrole needed to be integrated in succession using the knowledge gained in the synthesis of the individual components to provide the SIPN. 


\section{CHAPTER II \\ SYNTHESIS OF THE HYDROGEL COMPONENT}

\subsection{Introduction}

This chapter develops general observations and understanding of hydrogels. Several hydrogels were examined to understand the potential application and contribution toward the shape-changing conductive hydrogel desired. In keeping with maintaining high biocompatibility, polymerization and synthesis techniques minimizing the need for organics were utilized. UV polymerization was utilized to polymerize the hydrogels. Cross-linkers were examined and utilized in the hydrogels to promote structural elasticity and integrity. Microwave assisted reaction synthesis is to was the method used to synthesize the cross-linkers. The goal in this chapter is to discuss the development of the hydrogel component that would inherently maintain a usable matrix that can maintain structural integrity and have enough elasticity to permit swelling and potential shapechanging.

\subsection{Hydrogel Polymerization}

Acrylic Acid (AcA), acrylamide (AA), and methacrylamide (MA) were three hydrogel monomers examined. Their molecular structures of these monomers can be seen in Figure 12. 

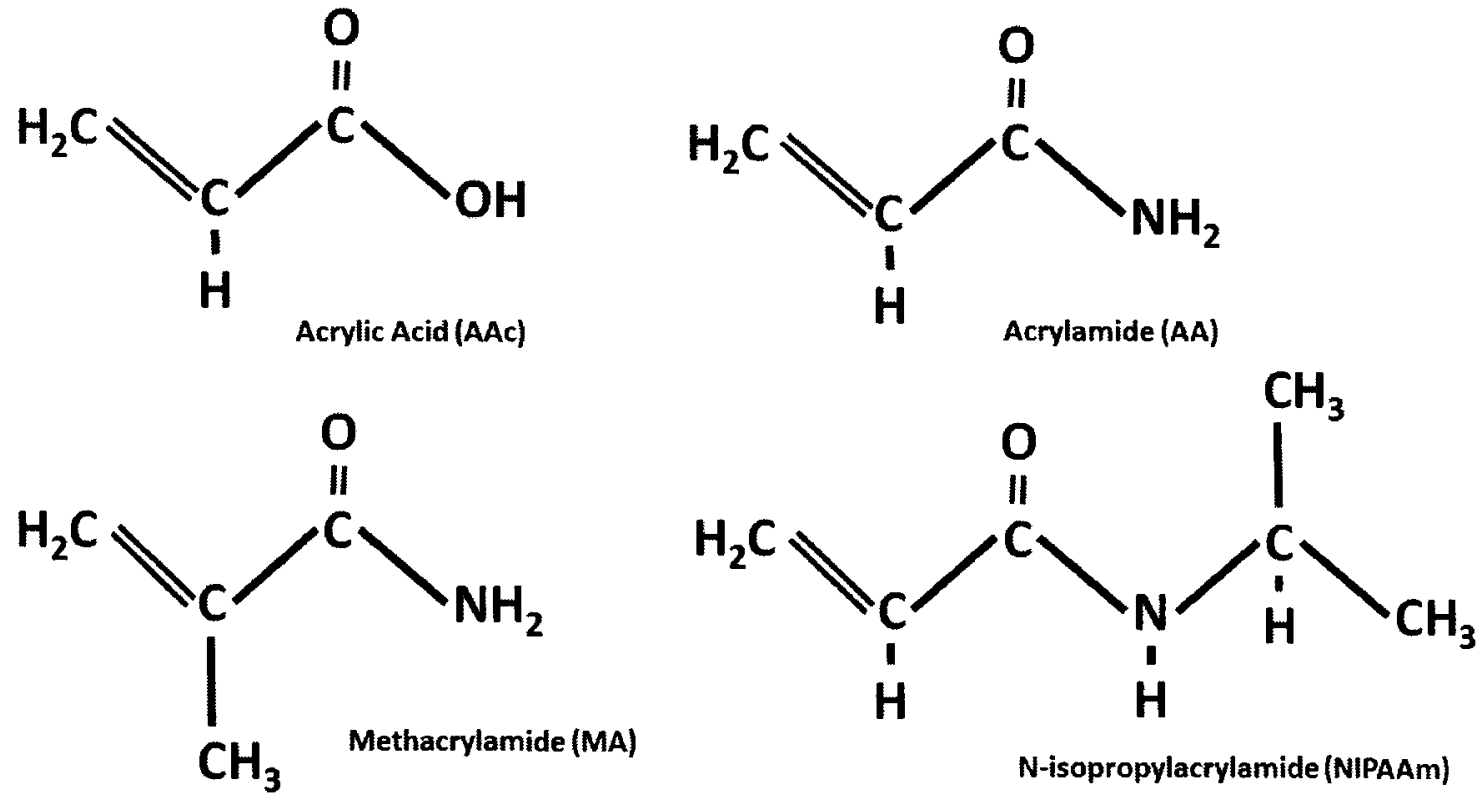

Figure 12. Molecular Structures of Several Hydrogels.

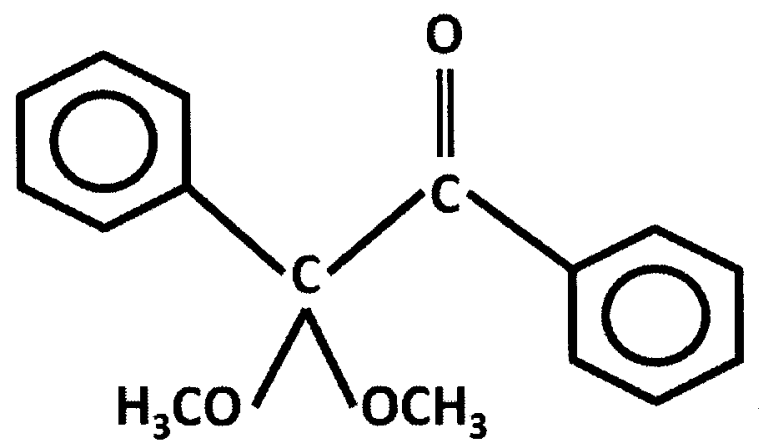

Figure 13. UV-Photo Initiator 2,2-Dimethoxy-2-phenylacetophenone (DMPA).

These three hydrogel monomers were examined based on their biocompatibility and established current use in biological applications. The initial studies were performed to increase and build a general understanding of photopolymerization and the 
homopolymers. Three different homopolymers were polymerized by ultraviolet (UV) photopolymerization using a UV source. Photopolymerization was chosen as the method of polymerization to minimize the use of organics in the polymerization reaction and increase biocompatibility. UV sensitive 2,2-Dimethoxy-2-phenylacetophenone (DMPA), illustrated in Figure 13, was used as a photoinitiator to promote free radical formation and polymerization of the monomers. Photoinitiators are chemical compounds that decompose into free radicals when exposed to light. These free radicals can be utilized to promote polymerization reactions without the need for toxic and expensive catalysts. Appendix A. Illustration of Photoinitiator Free Radical Generation illustrates the free radical generation from DMPA.

\subsubsection{Experimental Procedure - UV polymerization}

Three mixing schemes were used because it was determined that DMPA was more soluble in ethanol than water.

Scheme 1

- The monomer was mixed with various weight $\%$ of DMPA $(0.1-20 \%)$

- Monomer DMPA was dissolved in equal weight of D.I. water

Scheme 2

- Monomer was dissolved in ethanol

- DMPA was dissolved in ethanol

- Monomer solution and DMPA solution were mixed together into centrifuge tubes at various weight $\%$ of DMPA $(0.1-20 \%)$ 
Scheme 3

- Monomer was dissolved in D.I. water

- DMPA was dissolved in ethanol

- Monomer solution and DMPA solution were mixed together into centrifuge tubes at various weight $\%$ of DMPA $(0.1-20 \%)$

The monomer was dissolved in equal weight of deionized water or solvent and the photoinitiator, DMPA, was varied by weight $\%$ between 0.1 and $20 \%$ to the monomer. The monomer mixture was placed in an ultrasonic water bath (Branson 200, Fisher Scientific) for ten minutes to mix and dissolve the monomer into solution. The monomer solution was pipetted into either $0.6 \mathrm{ml}$ or $1.5 \mathrm{ml}$ polypropylene centrifuge tube and exposed to the UV source for ten minutes at $365 \mathrm{~nm}$ and an approximate intensity of 37.1 $\mathrm{mW} \mathrm{cm} \mathrm{cm}^{-2}$.

\subsubsection{Results \& Discussion}

Under Scheme 1, various amounts of DMPA in the mixtures with the monomers were examined to determine optimal reactions and complete polymerization. The weight percent of DMPA for monomers $\mathrm{AC}, \mathrm{AA}$, and MA were $0.1 \%,<0.1 \%$, and $5.0 \%$, respectively. Figure 14 shows photographs of the photopolymerized monomers. Both poly(acrylamide) and poly(methacrylamide) were soft and gel-like in texture and form, but poly(acrylic acid) was hard and had a glassy appearance. Increasing the amount of DMPA increased the rigidity of the homopolymer. 
All three of the hydrogels did not demonstrate conductivity. However, when these homopolymers were placed in water to determine swelling properties, the gels completely solvated, perhaps due to low cross-link density among the polymer chains.

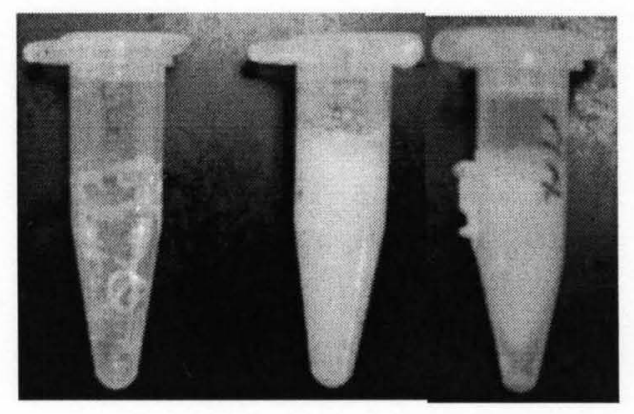

Figure 14. From left to right; AC, MA, and AA homopolymers.

Scheme 2 resulted in a white dry solid powder for the acrylic acid, a white colloid suspension for the methacrylamide. Scheme 3 resulted in viscous liquid gel. It was apparent that the photopolymerization was incomplete. In Figure 15 is visible that the acrylamide and methacrylamide are not clear or gel like as in Figure 14.

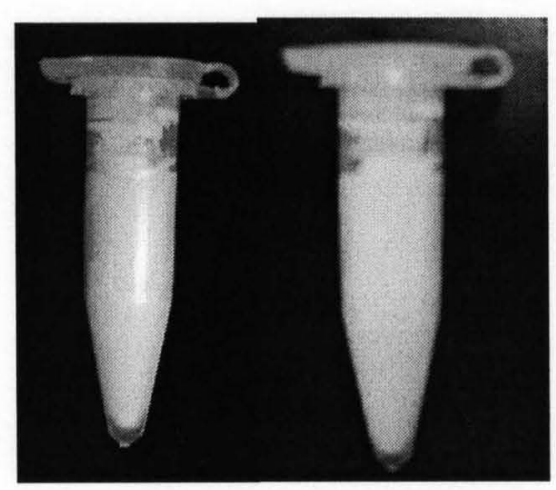

Figure 15. Polymerization of Acrylamide (Left) and Methacrylamide (Right) in Ethanol. 
The failure to photopolymerize in both Schemes 2 and 3 may be the result of the dielectric constant of the solvent. The dielectric constant is a relative measure of the polarity of solvent. The water value of the dielectric constant of 80.1 is very high relative to most other solvents. Solvents with dielectric constant less than 15 are generally considered non-polar [39]. Table 3 shows the properties between deonized water and ethanol.

Different solvents can have dramatic effects on the kinetics of many radical reactions [40]. For example, this work demonstrates how the photoinitiator 2-Methoxy2-phenylacetophenone, utilized in a photopolymerization reaction showed a reaction rate decrease of two orders of magnitude when the solvent changed from water to benzene [41]. The electron spin resonance spectrum of the free radical species is significantly different when in nonpolar solvents than in aqueous solvents [42]. In nonpolar solvents, the solvent may scavenge and quench the free radical. The solvent theoretically acts as a cage to surround the free radical and prevents accessibility by the monomers [43]. This may be a potential cause of why the monomers were not able to effectively polymerize under UV exposure.

\section{Table 3}

Dipole and Dielectric Properties of Some Solvents

Dipole Moment

Deionized Water

Ethanol

Benzene
1.85

1.69

0.0
Dielectric Constant

80.1

24.3

2.3 
In order to improve the structural integrity of the hydrogels polymerized in Scheme 1, it may be necessary to utilize a cross-linker to increase the cross-links within the hydrogel and improve its structural integrity in solutions.

Between the methacrylamide and acrylamide monomers, methacrylamide would be a slightly better monomer to use due to its slightly larger molecular size. The larger molecular size provides larger cross-links and higher swelling capability than what may be cross-linked with acrylamide.

\subsection{Cross-linker Synthesis}

The cross-links provide the structural gel formation of the hydrogel. The crosslink density can significantly modify the mechanical properties and physical behavior of the hydrogel. At low density the hydrogel maintains a low viscosity and the polymer can have fluidic properties. Intermediate cross-link densities allow the hydrogel to maintain elastomeric properties that allow swelling and flexibility. High cross-link densities cause the hydrogel to become rigid and glassy, and therefore more brittle.

Depending on the cross-link density, the cross-links have the potential to impact the conductivity of the polymer composite and the method by which the electrical current may be propagated throughout the hydrogel matrix. The swelling nature of the polymer composite may also be affected by the type and quantity of cross-links. Therefore, this has a significant potential to hinder or enhance the type of shape-change that can occur. For example, in a piezoelectric device, an applied force on the bulk material can produce a voltage. However, the spacing of the opposite charges or dipole density, which when 
changed or forced to misalign creates a higher potential energy within the bulk, is dependent on the microstructure of polymers [44].

Hydrogels can be used as cross-linkers between polymer chains to promote biocompatibility. Poly(ethylene glycol) dimethacrylate (PEGDMA) is a hydrophilic polymer that can be photopolymerized and is extensively utilized as a cross-linked hydrogel in biomaterials. Figure 16 below illustrates the molecular structure of PEGDMA.

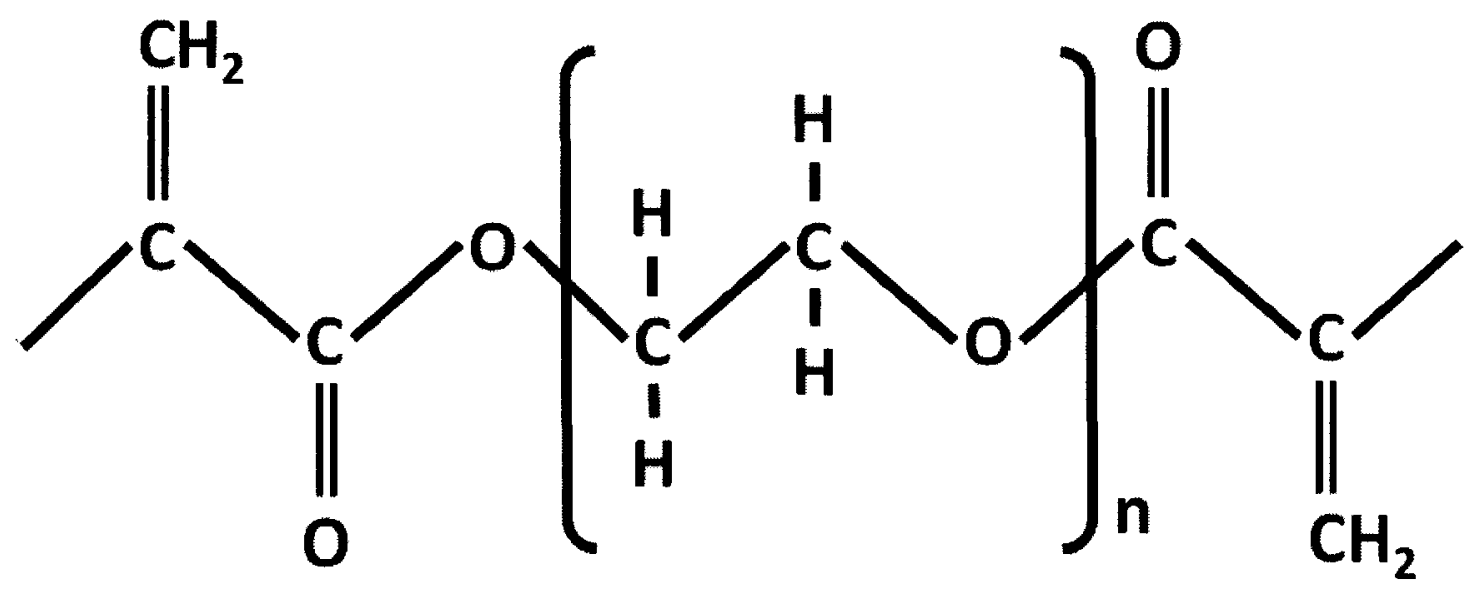

Figure 16. Polyethylene Glycol (PEG) Dimethacrylate Cross-linker.

\subsubsection{Microwave Assisted Synthesis Reaction for PEGDMA Cross-linker}

Lin-Gibson et al. demonstrated the fast and solvent-free formation of PEGDMA polymers utilizing microwave assisted reaction synthesis. Their comparison of the microwave assisted synthesis reaction to traditional organic synthesis techniques for PEGDMA demonstrated an alternative that involves fewer organic solvents and 
significantly decreases reaction times while still synthesizing effective cross-linker with high conversion between $82 \%$ and $93 \%$ [45]. Electromagnetic radiation can accelerate chemical reactions. Molecules with dipoles adsorb the electromagnetic radiation, converting the energy into heat with high efficiency so that superheating can be achieved at ambient pressure. Heating of the molecules occurs when the dipoles align under the magnetic field and agitation created by the reorientation of the molecules by the alternating magnetic field generated within the microwave. This technique also allows for the minimal use of organics during the synthesis of cross-linkers.

\subsubsection{Experimental Procedure - Microwave Assisted Synthesis Reaction}

PEGDMA polymers with 200 and 400 molecular weight PEG and with three and five-fold excess of methacrylic anhydride, were reacted in 30 second intervals up to an accumulated time of 5 minutes utilizing the microwave assisted polymerization. The reaction was performed in intervals to prevent the solution from overheating and boiling out of the glass vial. In between reaction intervals, the temperature was monitored with an infrared thermometer. The PEGDMA samples were then cooled to room temperature. Then, an ethyl ether volume of about 10 times the weight of the PEG reacted was added and the vial gently shaken to allow the PEGDMA to precipitate. The samples were placed in a freezer at $-20^{\circ} \mathrm{C}$ to facilitate the precipitation process. The precipitated PEGDMA samples were then filtered and dried. The PEGDMA samples were analyzed on high-resolution $400 \mathrm{MHz}$ proton NMR using deuterated chloroform as solvent at $10 \%$ polymer concentration by mass. 


\subsubsection{Results}

A minimum of at least a five-fold amount of methacrylic anhydride to PEG needed to be used to synthesize PEGDMA. To observe the effect on different PEG molecular weights as cross-linking agents, PEG200DMA and PEG400DMA were synthesized. Figure 17 illustrates the PEGDMA molecule and correlates the ${ }^{1} \mathrm{H}$ protons in the PEGDMA cross-linker to the proton peaks in ${ }^{1} \mathrm{H}$ NMR spectra shown in Figure 18 and Figure 19 show the ${ }^{1}$ H NMR spectra of the PEG200DMA and PEG400DMA crosslinkers synthesized, respectively. These spectra are similar to the ${ }^{1} \mathrm{H}$ NMR spectra published by Lin-Gibson et al. [45] and demonstrate that PEGDMA cross-linkers of various molecular weight PEG can be synthesized using the microwave assisted synthesis reaction technique.

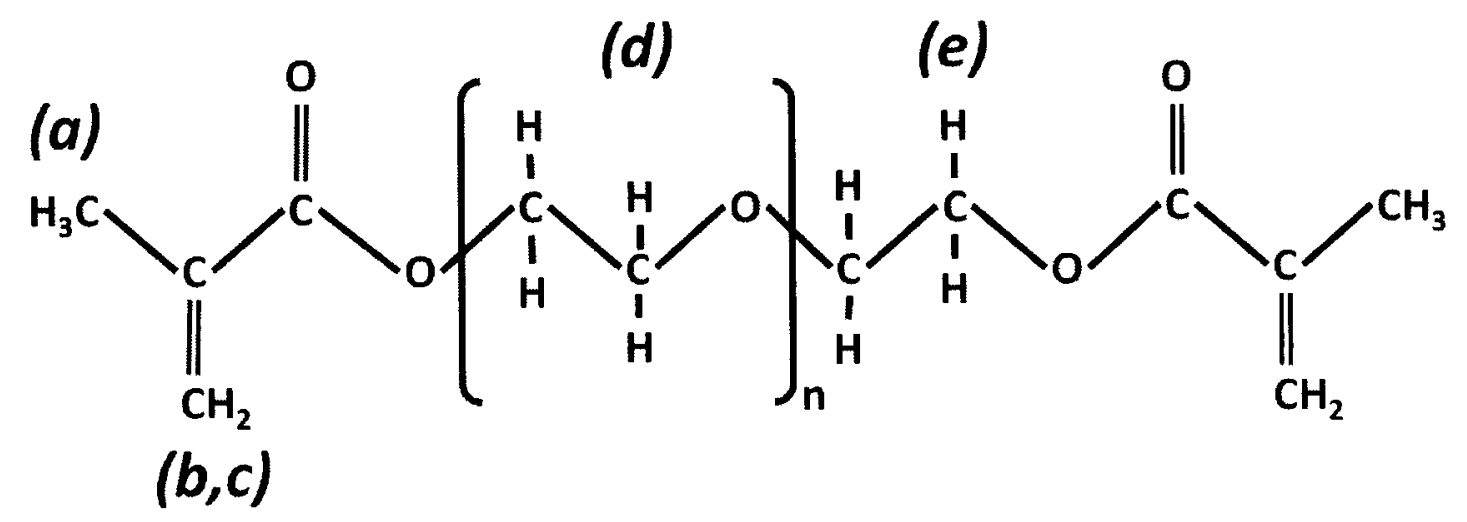

Figure 17. ${ }^{1} \mathrm{H}$ NMR peak correlation to PEGDMA Molecule. 


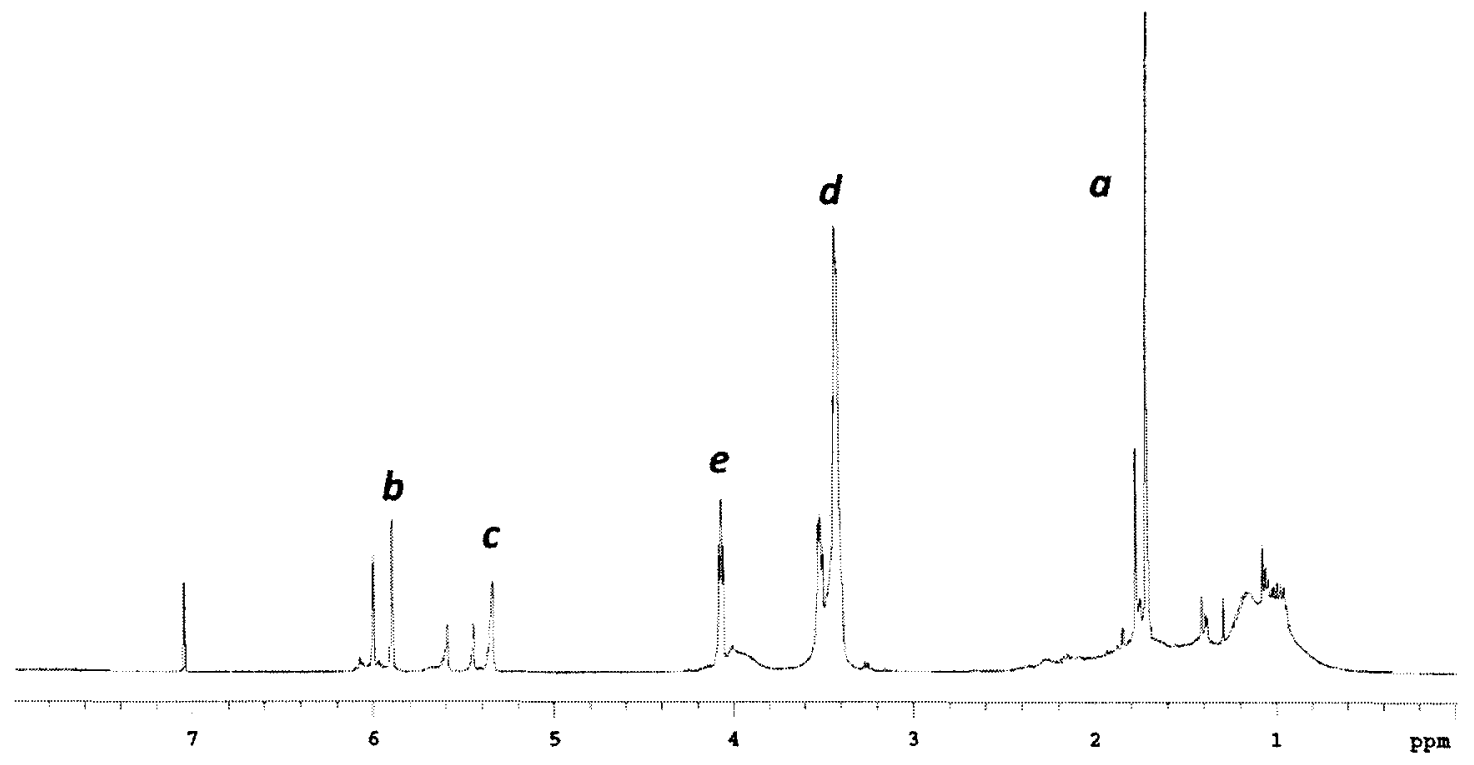

Figure 18. ${ }^{1} \mathrm{H}$ NMR of 200 Molecular Weight PEGDMA.

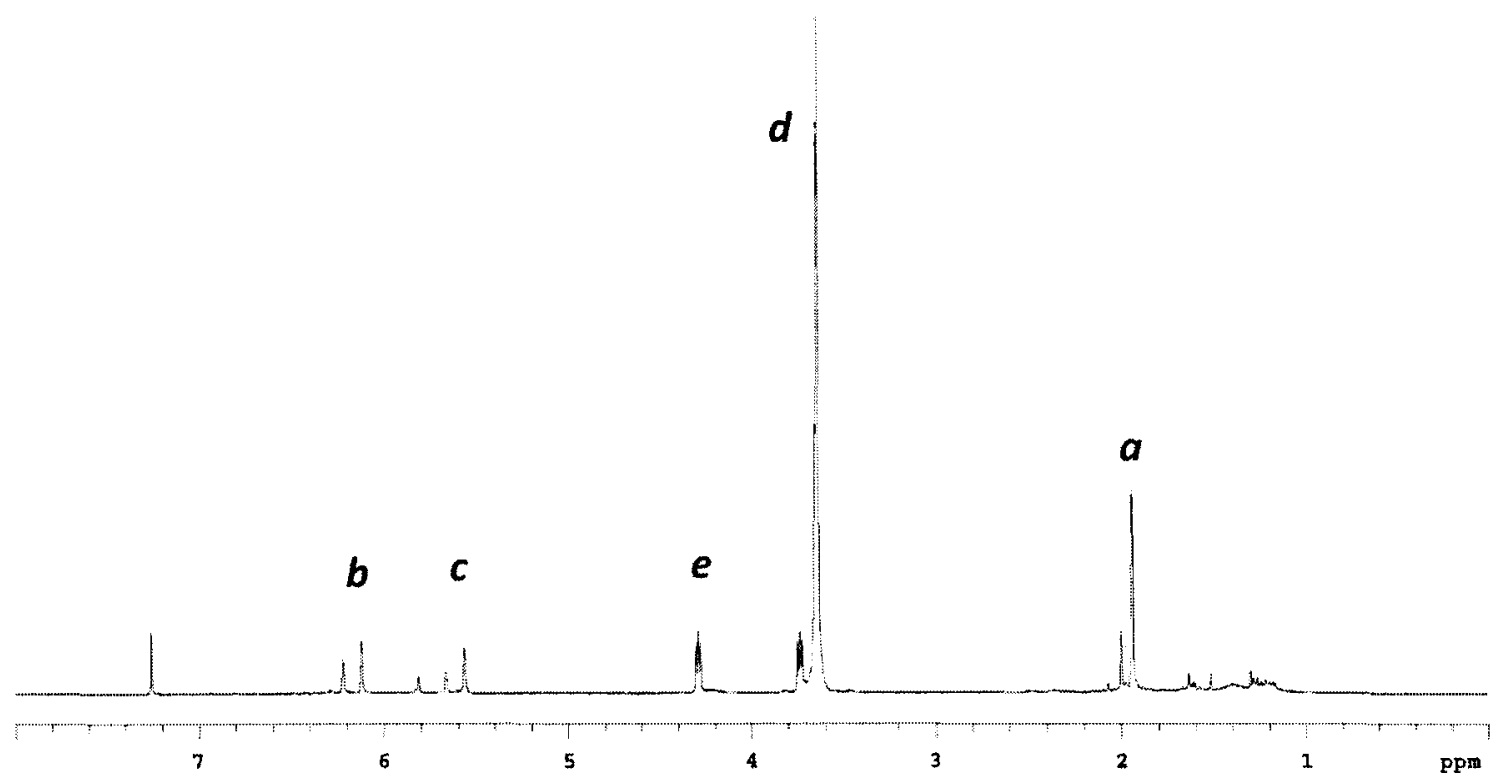

Figure 19. ${ }^{1} \mathrm{H}$ NMR of 400 Molecular Weight PEGDMA. 


\subsection{Hydrogel Cross-linker Polymerization}

The PEGDMA polymers were mixed with the methacrylamide monomer and photopolymerized just as the homopolymers were synthesized above to improve the structural property of the hydrogel. The hydrogel component was synthesized using UV photopolymerization with the cross-linker synthesized by microwave assisted synthesis.

\subsubsection{Experimental Method}

UV Polymerization of the methacrylamide monomer with various PEG molecular weights of PEGDMA cross-linkers were performed using Scheme 1 as outlined in Section 2.2.1 to determine the effect of the cross-linker molecular weight on the hydrogel. A $10 \mathrm{wt} \%$ of cross-linker by mass fraction was proportioned with the monomer. The DMPA photoinitiator was weight percent of $5.0 \%$, and the monomer and cross-linker mixture was exposed under UV for ten minutes at $365 \mathrm{~nm}$ and an approximate intensity of $37.1 \mathrm{~mW} \mathrm{~cm}{ }^{-2}$. The initial study of the PEGDMA cross-linkers used PEG molecular weights of 200,400 , and 550 .

\subsubsection{Results}

It was found that the synthesized PEGDMAs were not very soluble in deionized water and would not uniformly mix as a homogeneous liquid before photopolymerization. The methacrylamide cross-linked with PEGDMA and PEG 200 yielded a whiter and less transparent polymer. This material was stiffer and less flexible than the polymer with the PEG 550. The polymer with the PEG 550 was semi-transparent and more gel like in 
texture. Thus hydrogels with the PEGDMA were stiffer when synthesized with shorter molecular weight PEGs.

\subsection{Discussion}

The selection of solvent during photopolymerization of the hydrogel needed careful consideration. The quantity of photoinitiator and cross-linker utilized during the photopolymerization process were critical in determining the cross-link density. Crosslinkers with larger molecular weight PEG may be considered to promoted better elasticity.

These experiments showed how the hydrophilic properties of the gel may be increased by incorporating hydrogels as cross-linkers. Changing the length of the hydrogel cross-linkers tethering the monomers together into a copolymer may alter the physical structure of the gel.

Among the three hydrogels examined, methacrylamide maintained the highest pliability and soft structure and therefore was selected for the hydrogel. It was demonstrated that PEGDMA cross-linkers can be easily synthesized with varying PEG molecular weight. For these studies, 550- molecular weight PEGDMA, which was commercially purchased, was used as the cross-linker to provide consistency in hydrogel polymerization. It is critical that these hydrogels maintain a pliable and soft structure so that the polymer can properly swell and contract when it is actuated. 


\subsection{Biocompatibility Assay}

A preliminary protocol was developed utilizing L929 mouse fibroblast cells and the MTT assay. L929 mouse fibroblast cells are commonly used for material biocompatibility studies. These protocols include appropriate cell culturing methods for the L929 cells and the assay technique to assess the biocompatibility of the polymer samples using the MTT assay. This was an initial study to examine how effective the biocompatibility assay could show toxicity of the materials synthesized in this dissertation to cells. These studies were tested on acrylamide monomer, photo initiator, and a couple hydrogels cross-linked with PEG550DMA.

\subsubsection{Experimental Method}

Details for the methods and protocols for L929 mouse fibroblast cell growth and the protocol for the MTT assay can be found in Appendix B. Protocol for the Bioactivity Assay.. The cells were treated with a high (20:300) and low (1:300) concentration dilution of 1.0 M 2,2-Dimethoxy-2-phenylacetophenone (DMPA; UV-photo initiator) in ethanol, 5.0 M acrylamide in ethanol, poly(NIPAAM-550PEGDMA), and poly(acrylamide-550PEGDMA) for 24 hours inside the incubator. All samples were diluted with the growth media.

Two controls were established. One was a negative control for the growth media and a vehicle control for the ethanol used to dissolve the acrylamide and DMPA at a 10:300 dilution of ethanol in growth media. Ethanol was used as the solvating vehicle for the both acrylamide and DMPA because it provided better solubility for DMPA. 
The acrylamide monomer and DMPA were diluted in cell growth media at a high (20:300) and a low (1:300) concentration to exhibit possible toxic (high) and non-toxic (low) concentrations. It was also done in an attempt to demonstrate the effect of different concentrations of toxicity on the cells.

The hydrogels cross-linked with PEG550DMA were UV photopolymerized as thin films approximately $1 \mathrm{~mm}$ thick for these assays. The thin films were cut into small $3 \times 3 \mathrm{~mm}$ squares. The hydrogels were immersed in deionized water and sterilized in a steam autoclave@ $121^{\circ} \mathrm{C}$ for $20 \mathrm{~min}$. The hydrogels were tested in two different protocols. In one, the hydrogel square was placed in $300 \mu$ of media for 24 hours in attempt to extract any organics that may leach from the hydrogel. This leachate media was then placed in the microplate wells for 24 hours of incubation with the cells. In the second, protocol the hydrogel was placed in the well during the first 24 hours of incubation. This was done to determine if there was any effect of the hydrogel physically on top of the cells.

The cells in the 96-well plate were treated with the samples in triplicate for at least 24 hours @ $37^{\circ} \mathrm{C}$. In order to monitor any possible cell death following treatment of the cells, the samples were removed and replaced with growth media to allow possible growth for another 24 hours. Therefore, this study examined two different time periods for cell death: $24-0,24 \mathrm{hr}$ treatment followed by immediate MTT assay; and 24-24, replaced with growth media following treatment and assayed 24 hours later. 


\subsubsection{Results and Discussion}

The preliminary results in Figure 20 show that the ethanol vehicle control shows that a small diluted amount of ethanol in the growth media would probably not be significantly toxic to the cells. The viability of the cells with ethanol was comparable to that of the negative control. Therefore, ethanol may be used as a vehicle at the low concentrations observed to test monomers and other chemicals in the polymer that need a solvent. A positive cytotoxic reaction of samples on the L929 cells is shown in an image in Figure 21. The illustration shows the appearance of grainy and lack normal

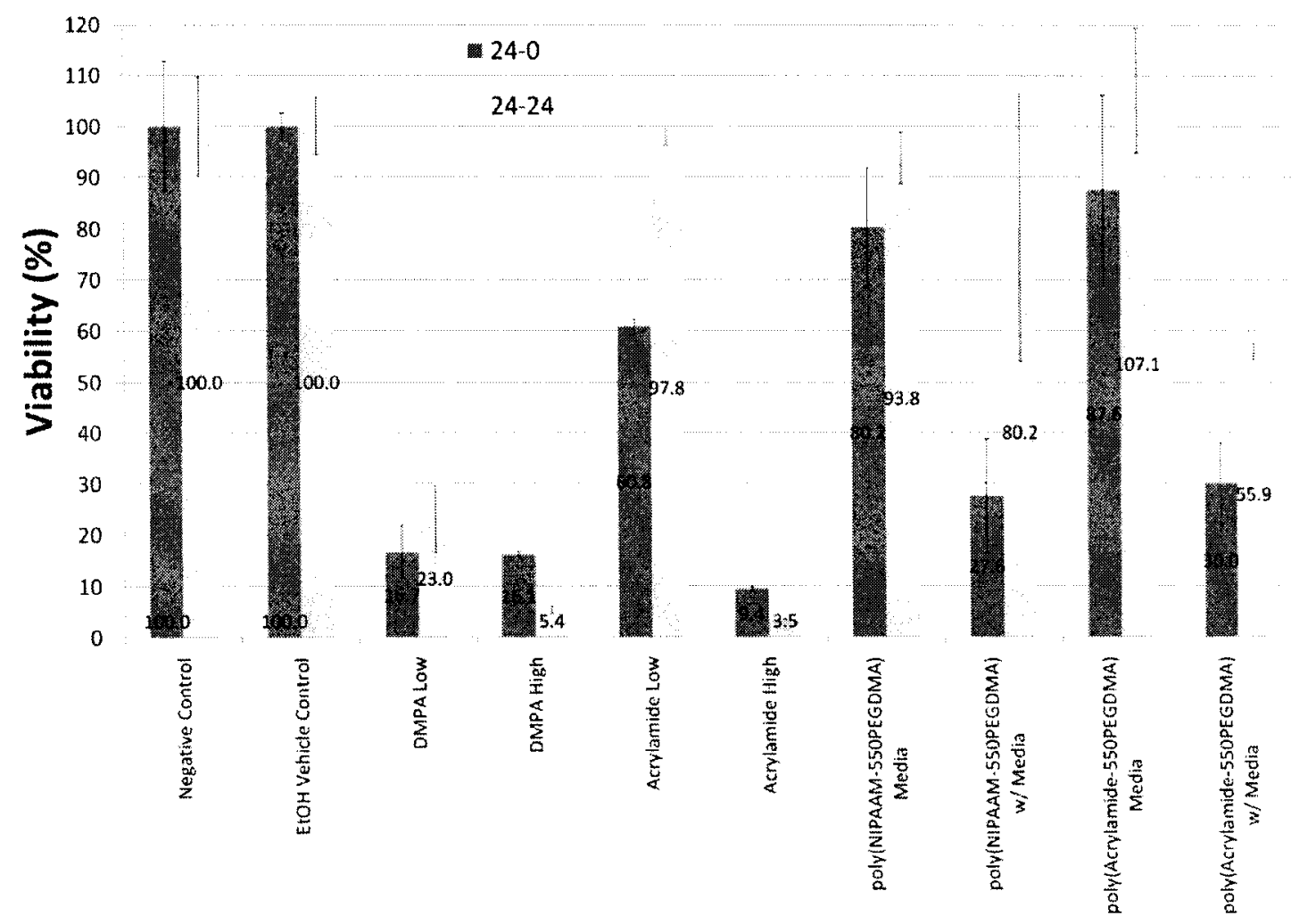

Figure 20. Preliminary results of cell viability from MTT assay of hydrogel samples demonstrating biocompatibility. 


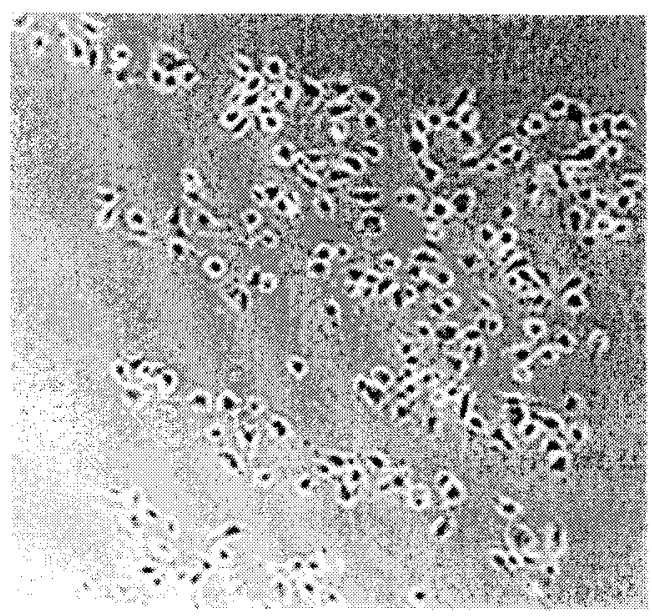

Figure 21. Image of a positive cytotoxic reaction on cell viability following treatment.

cytoplasmic space if the treatment is toxic to the cells. There are considerably more open areas between cells which indicate that extensive cell lysis have occurred. The treatment of acrylamide and DMPA demonstrated different levels of toxicity to the cells at the diluted concentrations, especially at low concentrations. At the low concentration, it was evident that DMPA is significantly more toxic to cells than acrylamide. It is important to identify the concentration that will produce a toxic response of the cell. At high concentrations it was demonstrated how acrylamide and DMPA can be toxic to the cells.

The poly(NIPAAM-550PEGDMA) and poly(acrylamide-550PEGDMA) samples left in media both showed some little cytotoxicity to the cells. This indicated that there may be some leaching of organics from the hydrogels that could potentially cause toxicity over extended time. The cross-linked hydrogels however demonstrated that they may be very highly biocompatible when compared to the negative control. The hydrogel samples placed directly on top of the cells showed a significant decrease in cell viability 
in comparison to the hydrogel leachate samples. This demonstrated how the decrease in cell viability may be due to the stress of the polymer squares sitting on top of the cells.

The results between the 24-0 and 24-24 plate of the MTT assay in Figure 20 also demonstrate that the cells will need some time after treatment to allow those cells affected by toxicity from the sample to die. Toxicity and cell death were not immediate and 24 hour period following sample treatment should be necessary to show the true effect of the samples on the cells. This initial study shows promise to determine biocompatibility of our material.

\subsection{Deductions on the Synthesis of the Hydrogel}

It was demonstrated that the hydrogel can be synthesized by UV polymerization with a cross-linker synthesized by microwave assisted synthesis reaction. However, the hydrogel needs a secondary element introduced into its matrix to provide it with conductive properties. Based on the selection of the hydrogel and cross-linker, the next step was to establish the synthesis of the conductive component of the polymer compound. Conductive elements may be provided by either doping the compound with gold nanoparticles or by introducing a second polymer with conductive properties. 


\section{CHAPTER III}

\section{CONDUCTION ENHANCEMENT WITH GOLD NANOPARTICLES}

\subsection{Introduction}

This chapter advances the hydrogel polymerized in the previous chapter by observing the potential of enhancing conduction by utilizing gold nanoparticles (GNPs). It was apparent from the results discussed in the previous chapter that the hydrogels were not conductive. Our intent was to synthesize a material that is conductive so that it can provide a larger and faster response than that provided by hydrogels that require the diffusion of electrolytes into and out of the hydrogel crosslinks. Gold was selected because it is a conductive metal that is biocompatible. It has been observed that the addition only $0.2 \mathrm{wt.} \%$ of GNPs decreased the resistivity of poly (methyl methacrylate) by $40 \%$ [46]. This may decrease the power necessary to actuate the copolymer.

\subsection{Experimental Scheme}

Gold nanoparticles were synthesized using precipitation reaction of gold chloride solution $\left(\mathrm{HAuCl}_{4}\right)$ and sodium citrate. A $1.0 \mathrm{mM}$ concentration of $\mathrm{HAuCl}_{4}$ was heated to boiling with a magnetic stir bar. After boiling began, a $38.8 \mathrm{mM}$ concentration of $\mathrm{Na}_{3} \mathrm{C}_{6} \mathrm{H}_{5} \mathrm{O}_{7}$ (sodium citrate) was added. As the solution continued to boil the sodium citrate would reduce the Au ions to nanoparticles of Au metal. throughout the boiling 
process, distilled water was added to maintain constant liquid volume. The excess citrate anions would stick to the Au metal surface to act as a surfactant and give each $\mathrm{Au}$ nanoparticle a negative charge. The solution would turn a deep red color to indicate the formation of the Au nanoparticles. Figure 22 shows an AFM image of the synthesized gold nanoparticles, which were about $40 \pm 5 \mathrm{~nm}$.

Gold nanoparticles were doped into the poly(acrylamide) homopolymer in an attempt to increase conduction. Poly (acrylamide) was doped by adding the gold nanoparticle solution in to the monomer solution as prepared in Scheme 1 for the UV polymerization procedure in Section 2.2.1. The poly(acrylamide) was doped with 0.1 and $0.2 \mathrm{wt} \%$ of the gold nanoparticles. The acrylamide monomer and gold nanoparticle solution was then placed under the UV lamp for photopolymerization. The first several hydrogel and gold nanoparticle composites were photopolymerized in $1.5 \mathrm{ml}$ centrifuge tubes as shown in Figure 23.

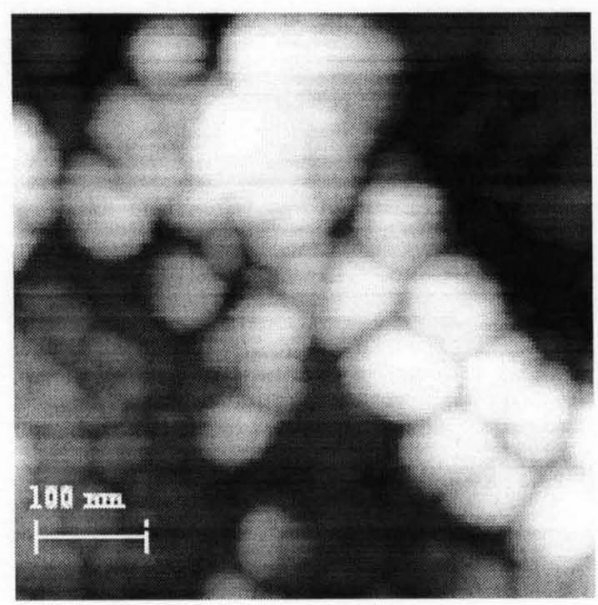

Figure 22. AFM Image of Au Nanoparticles. 


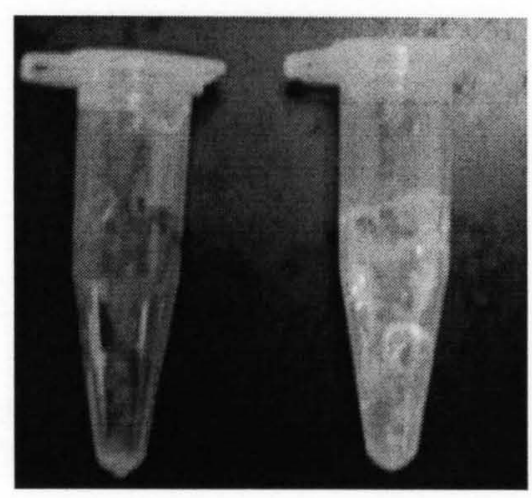

Figure 23. Poly(acrylamide); Au doped (left) and undoped (right).

\subsection{Result \& Discussion}

Figure 23 shows a photograph of the poly(acrylamide) with and without gold nanoparticles. The red tint makes the presence of the gold nanoparticles within the hydrogel clearly visible. However, the poly(acrylamide) doped with the gold nanoparticles showed no observable increase in electrical conductivity. The hydrogel and gold nanoparticle composites were polymerized in thin films in an attempt to concentrate the gold nanoparticles in at least one direction. It was believed that this may improve the conductivity of the composite by partially concentrating the gold nanoparticles in the hydrogel. The thin films were formed by pipetting the monomer and gold nanoparticle solution into a 1" x 1" silicone mold that was $1 \mathrm{~mm}$ deep. Conductivity measurements on this thin film did not show any observable increase in conductivity.

Agglomeration of the gold nanoparticles may have possibly prevented their even distribution of the particles throughout the polymer. The size and dispersion of the $\mathrm{Au}$ nanoparticles within the hydrogel can be seen within the SEM images in Figure 24 and Figure 25. The gold nanoparticles in these figures are highlighted as bright white specks 
throughout the image. It may also be possible that the concentration or density of the gold particles within the hydrogel was not high enough to provide the plasmon field necessary to provide conduction between the GNPs. It may also be a possibility that the size of the GNPs were too large to reduce the plasmon field generated by smaller gold nanoparticles. The effective number of surface atoms on the gold nanoparticle can drastically increase by decreasing the size of the nanoparticles for a given bulk amount of the material [47].

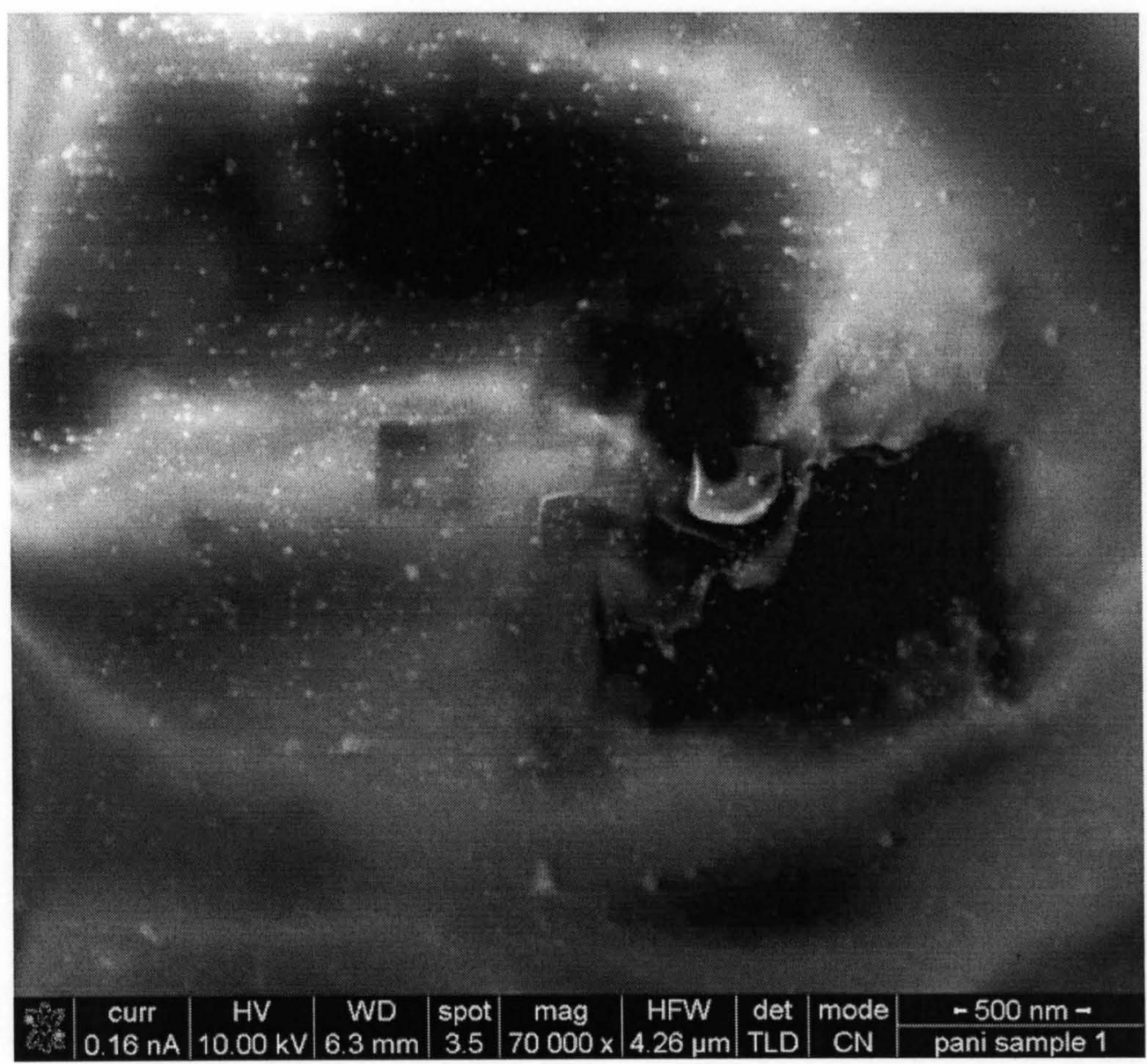

Figure 24. SEM images of gold nanoparticles (GNPs) dispersed in poly(acrylamide) at $500 \mathrm{~nm}$ scale. 


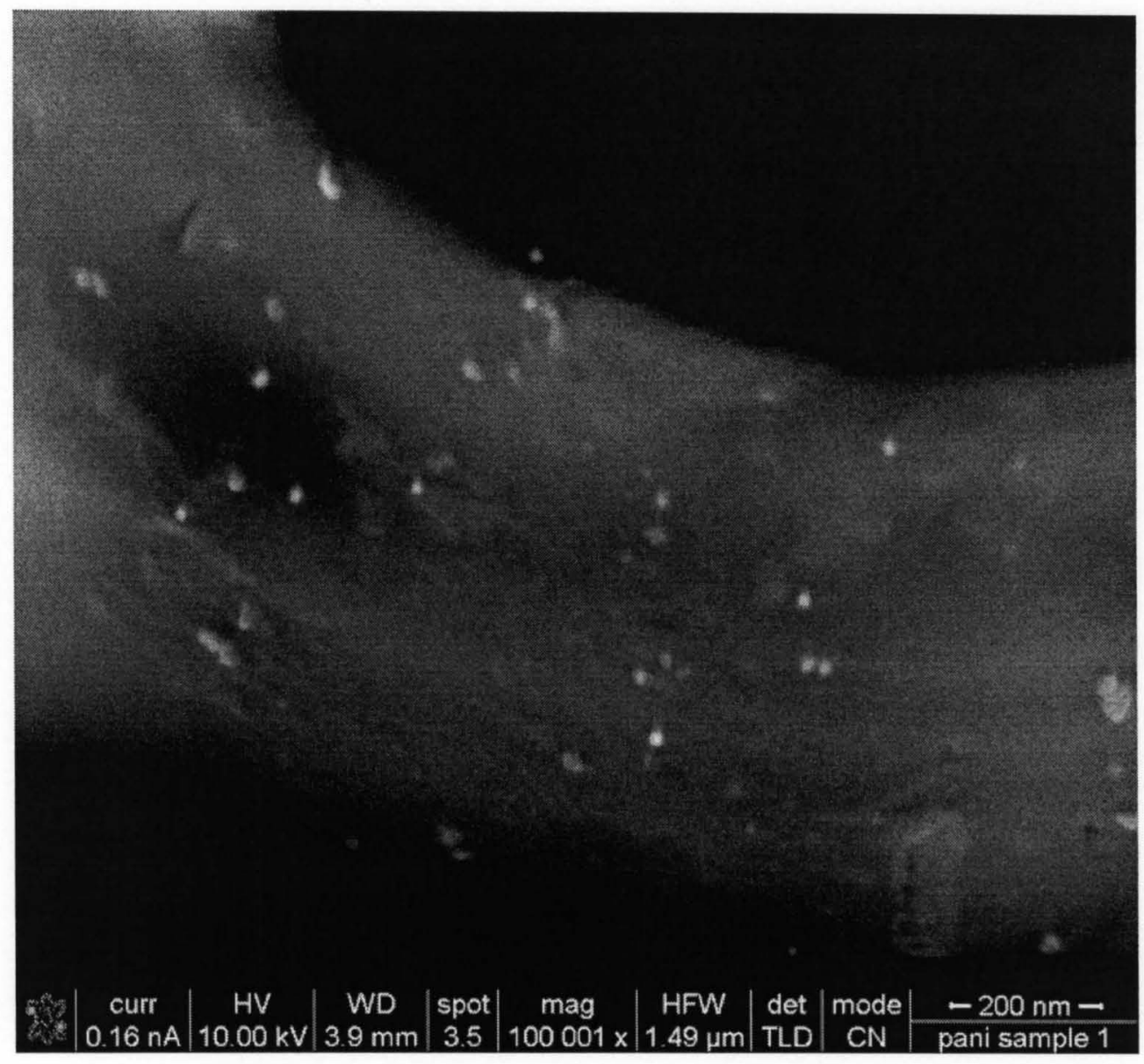

Figure 25. SEM images of gold nanoparticles (GNPs) dispersed in poly(acrylamide) at $200 \mathrm{~nm}$ scale.

Doping the copolymer with nanoparticles may change its physical properties as well. It has been shown that the addition of nanoparticles into the hydrogel can decrease their swelling ratio [48]. The hydrogel doped with gold nanoparticles seemed to be more brittle than the hydrogel without the gold nanoparticles based on the direct observation between the two. It was evident that the poly(acrylamide) doped with the gold nanoparticles was less flexible as the poly(acrylamide) that was not doped. The doped 
poly(acrylamide) would crack and break apart when folded in half, whereas the undoped poly(acrylamide) could bend without cracking.

In Figure 25, agglomeration of some of the gold nanoparticles is evident by several white specks that are lumped together in an irregular shape. It may be possible to improve the distribution of the gold nanoparticles and minimize agglomeration by homogeneous nucleation of the gold nanoparticles as the hydrogel is photopolymerized. By nucleating and forming the particles in situ, it may be possible to also reduce the size of the gold nanoparticles.

Based on these observations, the utilization of GNPs may not be the most suitable method to enhance the conduction of the hydrogel. The conductivity of the hydrogel may be improved with the addition of large amounts of GNPs but it may be with the compromise to the flexibility of the hydrogel. An alternative material that provides conductivity needed to be considered in the design of this polymer compound. The alternative material to be examined to provide conductivity was namely in the area of conductive polymers. 


\section{CHAPTER IV}

\section{SYNTHESIS OF ELECTROACTIVE COMPONENT}

\subsection{Introduction}

Hydrogels do not have inherent conductive properties. The doping of gold nanoparticles into hydrogels demonstrated that there may be a sacrifice of material flexibility over potential increase in conductivity. Conductive polymers are a class of materials that typically provide the deforming mechanical properties as a response to electrical stimulation. Polypyrrole is conductive polymer that may be polymerized to contribute to the conductive and mechanical properties. In this chapter, we will discuss the different polymerization methods for polypyrrole that were investigated based on the needs of combining these materials with the hydrogels to meet the goals of this project.

\subsection{Electroactive Polymers}

Electroactive polymers (EAPs) are conducting polymers which can respond to electrical stimulation by a significant deformation of size or shape. EAPs evolved from the discovery of piezoelectric polymers, such as polyvinylidene fluoride (PVDF) which has the ability to generate electric potential upon application of a stress field [49]. A reverse piezoelectric effect produces stress or stain when a negative electric field is applied. EAPs have a negative piezoelectric modulus $\left(\mathrm{D}_{33}\right)$ which causes the material to 
compress instead of expand under an electric field [50]. The most recent examples of hydrated electric EAPs require relatively low voltages $(<10 \mathrm{~V} / \mathrm{m})$ to achieve bending deflections greater than $90^{\circ}[51]$.

The two major mechanisms of activating EAPs are through ionic or electrical potentials. Electric EAPs are actuated by electric fields or Coulomb Forces whereas ionic EAPs are actuated by potentials created through the diffusion of ions. Ionic EAPs require only low voltages to initiate ionic diffusion and actuate predominately in bending motions with large displacements. Changing the polarity of the applied voltage allows for bi-directional actuation in ionic EAPs because it is dependent on ionic diffusion. However, a major disadvantage is that few ionic EAPs can hold strain under DC voltage. In addition, most respond slowly, induce a relatively low bending actuation force, require the use of an electrolyte, cannot be operated in open air conditions, have a low electromechanical coupling efficiency, and degrade in aqueous systems at voltages greater than $1.23 \mathrm{~V}$.

The advantages of electric EAPs are that they can operate at room temperature conditions for long periods of time, have rapid response times in the $\mathrm{mSec}$ range, can hold strain under DC activation, exhibit high mechanical energy density, and induce relatively large actuation forces [31]. Electric EAPs generally require high voltages $(\sim 110 \mathrm{MV} / \mathrm{m})$, and are independent of voltage polarity. A recent development in ferroelectric EAPs allows for $\sim 20 \mathrm{MV} / \mathrm{m}$ actuation though it does require compromise between strain and stress. Most actuation is monopolar due to associated electrostriction effects; and the glass transition temperature is low for actuation tasks, which results in a very brittle material [52]. 


\subsection{Pyrrole}

Pyrrole is a heterocyclic aromatic organic compound. It is a five-membered ring with a lone pair of delocalized electrons on the nitrogen within the aromatic ring. Pyrrole predominately undergoes electrophilic aromatic substitution at the 2 and 5 positions, as illustrated in Figure 26. Pyrrole is photosensitive and undergoes polymerization in light or by oxidizing agents. It is a volatile liquid that, like many amines, darkens upon exposure to air and light. It must be distilled to remove impurities such as polypyrrole. Pyrrole is highly activated due to the presence of an unshared pair of electrons on the nitrogen. This increases the affinity of the pyrrole molecule for cationic intermediates.
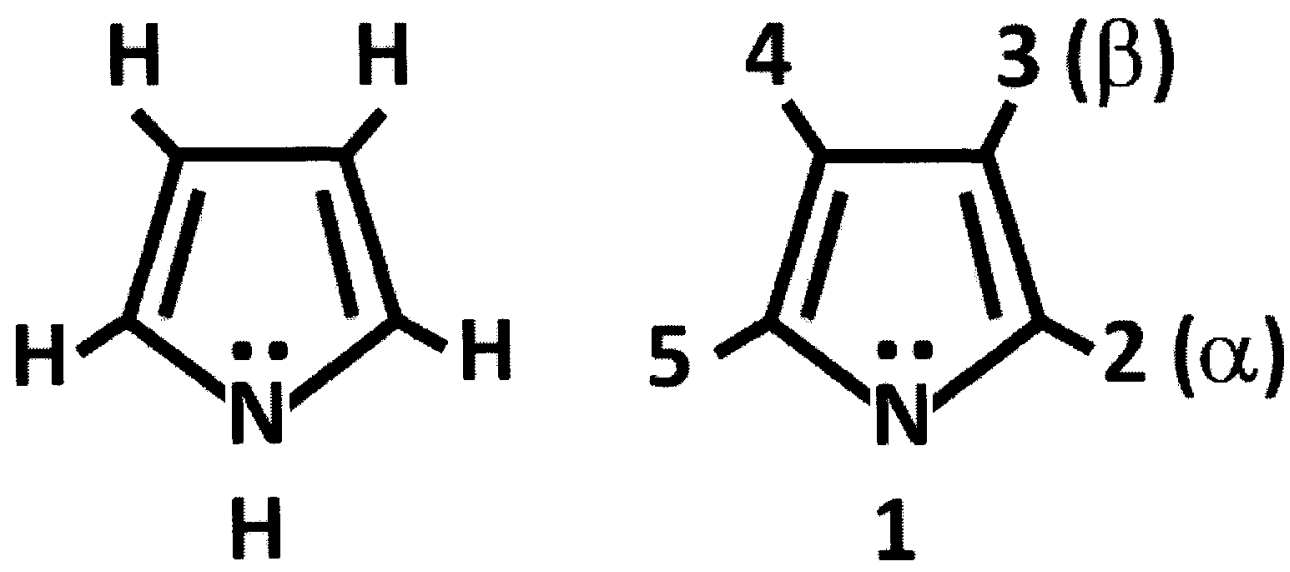

Figure 26. Pyrrole Molecule.

The best geometry for pyrrole is in the sp2-hybridized configuration with the $\mathrm{N}$ in a triagonal planar position. When $\mathrm{N}$ is sp3-hybridized it forms a tetrahedral structure with five valence electrons in the four sp3 orbitals, three $\sigma$ bonds, and one non-bonded 
pair of electrons. When $\mathrm{N}$ is sp2-hybridized it forms 3 valence electrons in the three sp2 orbitals of the $\sigma$ bonds, and leaves 2 valence electrons in the hybridized p orbital where the electrons are more mobile. This is illustrated in Figure 27.
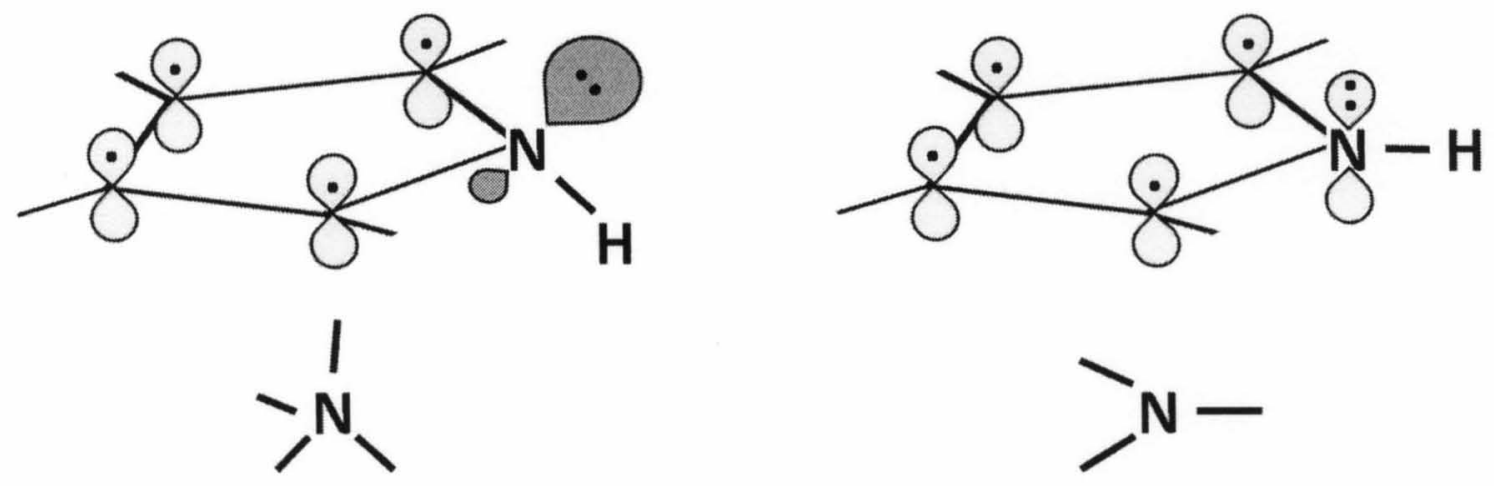

Figure 27. sp3 (left) and sp2 (right) hybridization of pyrrole.

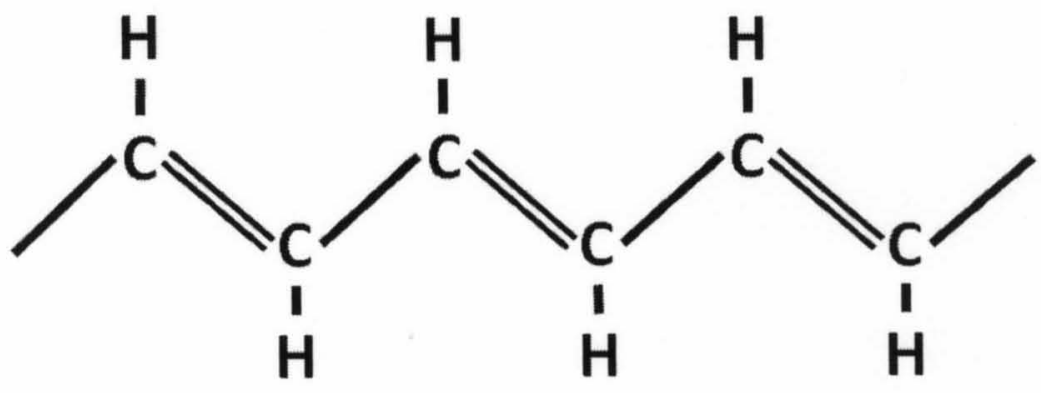

Figure 28. Polyacetylene molecule. 


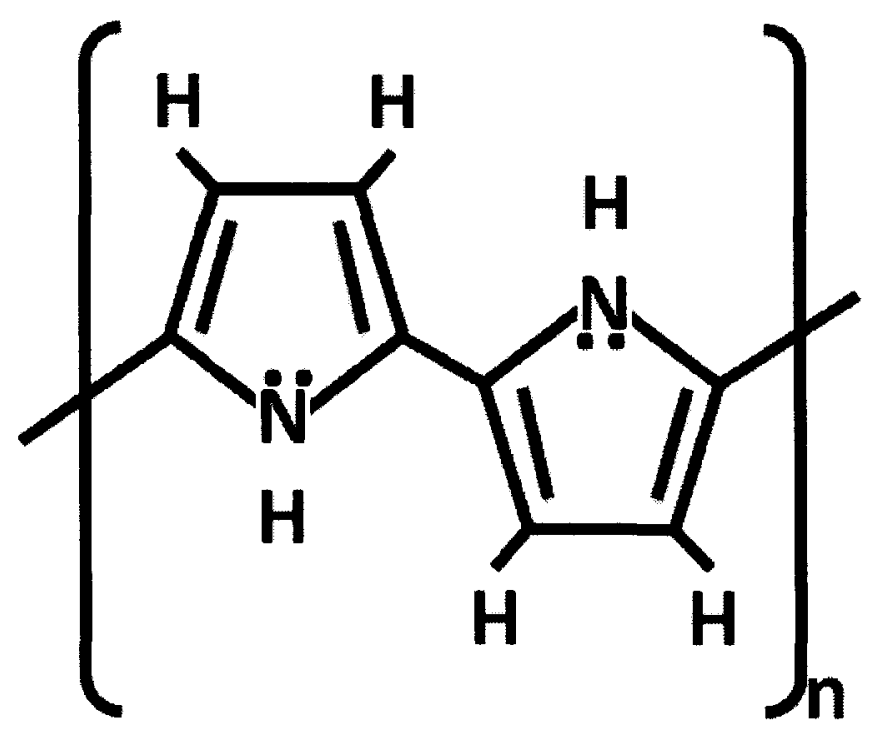

Figure 29. Polypyrrole repeating unit in polymer chain.

Polypyrrole is a derivative of polyacetylene, whose high electrical conductivity is due to the long chain of carbon atoms with alternating single and double bonds as illustrated in Figure 28. Much like polyacetylene, the alternating single and double bonds in polypyrrole gives it conductive properties, which is illustrated in Figure 29. Although its conductivity is not as high as polyacetylene, it was the first polyacetylene-derivative to show high conductivity. Iodine-doped, oxidized, polypyrrole displayed conductivity as high as $1 \times 10^{2} \mathrm{~S} / \mathrm{m}$ [53]. Pyrrole is commonly used in biosensors because it is biocompatible and it easily immobilizes various biologically active compounds [54].

\subsection{Electrochemical Polymerization}

Electropolymerization is frequently utilized for the polymerization of pyrrole. Potential is applied across an electrolyte solution containing pyrrole to polymerize a 
polypyrrole film on the anode. Galvanostatic polymerization and potentiostatic polymerization are the two common electropolymerization setups carried out at constant current or constant voltage, respectively. Galvanostatic polymerization at constant current was the chosen method for this work because it allows for better control of film thickness and porosity [55].

Figure 30 shows a schematic of the electrochemical polymerization cell that was used. Several groups have demonstrated that polypyrrole can be electropolymerized on indium tin oxide (ITO) $[56,57,58]$ rather than on glassy carbon or platinum. Indium tin oxide $\left(1 \mathrm{x} l \mathrm{in}^{2}\right), 5 \mathrm{~cm}$ coiled platinum wire, and $\mathrm{Ag} / \mathrm{AgCl}$ electrode was initially utilized as the working electrode (WE), counter electrode (CE), and reference electrode (RE), respectively. An electrochemical cell volume of about $30 \mathrm{ml}$ was chosen to minimize the amount of materials used. Electropolymerization of pyrrole using various electrolyte solutions were examined.

\subsubsection{Experimental Method - Electrochemical Polymerization}

The electrochemical cell was connected to a VSP Potentiostat/ Galvanostat with EC-Lab Software to control the chronoamperometic technique. Electropolymerization reactions were performed in an anaerobic environment under a $\mathrm{N}_{2}$ blanket at a current density of $\sim 0.1 \mathrm{mV} / \mathrm{cm} 2$. 


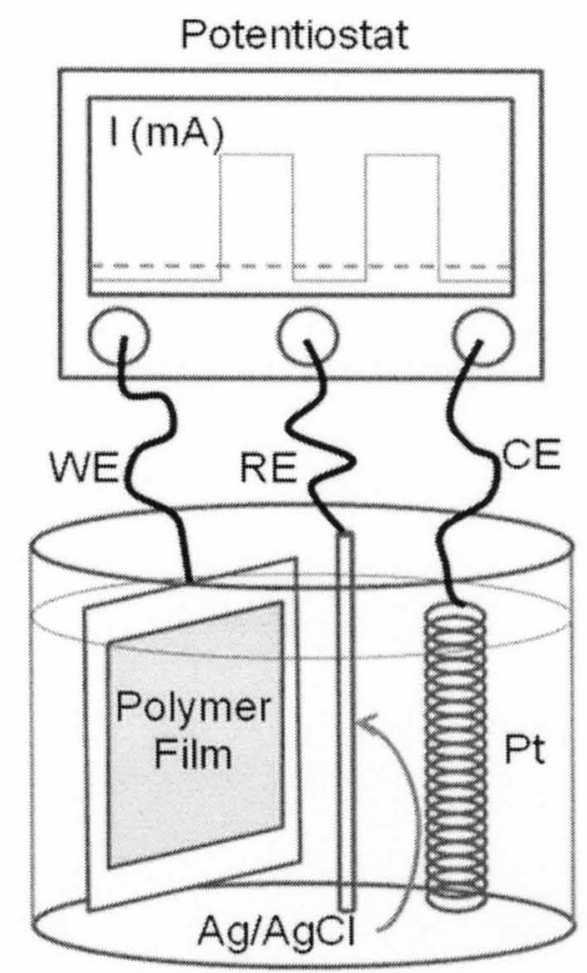

Figure 30. Illustration of electrochemical cell

Factors affecting electropolymerization are the electrolyte solution, current density, temperature, and reaction time. However for this study, only electrolyte conditions based on literature solutions with $0.1 \mathrm{M}$ pyrrole were studied:

(1) $0.1 \mathrm{M} \mathrm{p}$-toluenesulphonic acid sodium salt (pTS) [56,59], room temperature.

(2) $0.05 \mathrm{M}$ tetrabutylammonium hexafluorophosphate (TBA PF6) in polypylene carbonate (PC) and $1 \%$ vol. DI water, $4^{\circ} \mathrm{C} . \quad[60,61,62]$.

(3) $0.05 \mathrm{M}$ tetraethylammonium hexafluorophosphate (TEAP) in propylene carbonate (PC) with $1 \%$ vol. DI water, $4^{\circ} \mathrm{C} . \quad[63,64,65,66,67,68]$. 
The literature solution conditions and electrochemical cell setups are tabled in Appendix C: Table of Literature Citations for the Setup and Conditions for the Electropolymerization of Polypyrrole. Both the pyrrole and PC were distilled prior to use. Yamaura's setup and procedure methods were followed for the electropolymerization [69]. Polypyrrole films were prepared by anodic oxidation of pyrrole in an electrolyte solution in an electrolytic cell. In our electrolytic cell, a coil platinum wire was used as the anode, and an Indium Tin Oxide (ITO) substrate was used as the cathode, which provided an area at least ten times as large as the anode. The reaction was carried out under constant current and a nitrogen blanket.

Yoon et al. and $\mathrm{Hu}$ et al. noted that the temperature of the electrolyte could influence the conductivity and capacitance of the polypyrrole $[70,71]$. Hence, initial studies were performed at room temperature and at $4^{\circ} \mathrm{C}$ to determine the magnitude of the effect of electrolyte temperature. To maintain $4^{\circ} \mathrm{C}$ temperature of the electrolyte solution, the electrochemical cell was placed in a refrigerant gel while the temperature was monitored by an infrared thermometer.

\subsubsection{Result \& Discussion}

All three electrolyte solutions led to the formation of polypyrrole (PPy) films. Figure 31 shows images of the PPy that was electropolymerized in the ITO glass using TEAP as the electrolyte. The PPy thin films showed conductivity but the polymer was brittle and not mechanically responsive to the application of an electrical current. Electropolymerization of polypyrrole at room temperature and $4^{\circ} \mathrm{C}$ did not result in significant difference in the conductivity of the PPy film. 

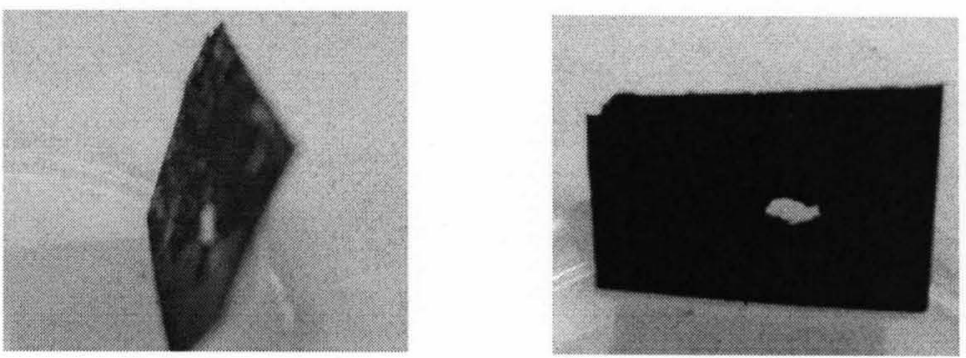

Figure 31. Photos of Polypyrrole electropolymerized on ITO substrate.

A FTIR analysis was done on the polypyrrole sample polymerized on the ITO substrate in Figure 31. To prepare the samples the hydrogel was pulverized into a power and a mull of the pulverized polymer powder with mineral oil was used in the FTIR analyzer. The mull was smeared on a $\mathrm{AgCl}$ plate and analyzed. Figure 32 shows the FTIR spectra of the polypyrrole in the mineral oil mull. The IR absorption peaks for

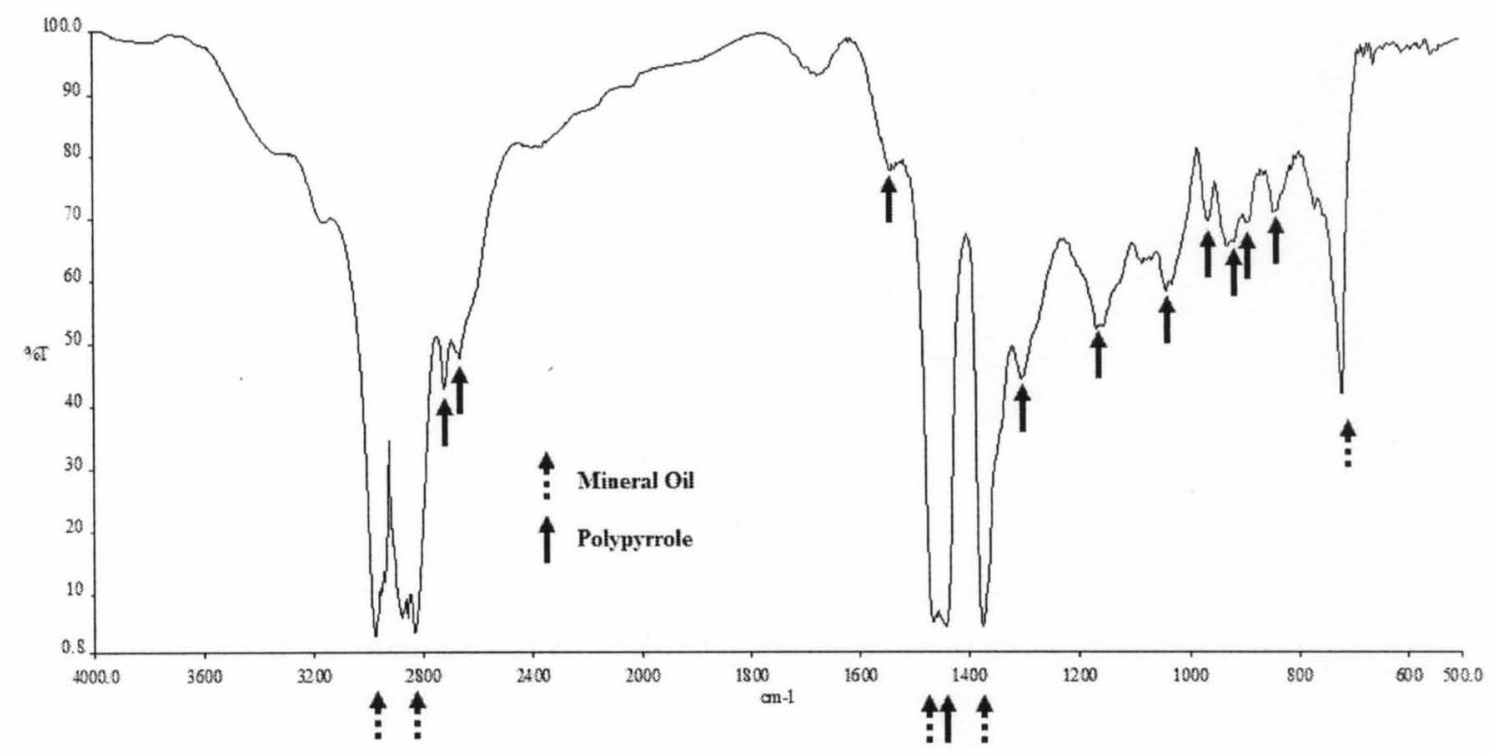

Figure 32. FTIR spectra of electropolymerized PPY in mineral oil mull. 
mineral oil are noted with arrows at 2920,2850,1470,1380, and $720 \mathrm{~cm}^{-1}$ and correlate with literature values [72]. The arrows marking the IR adsorption peaks for polypyrrole in Figure 32 at 2720, 2670, 1547, 1487, 1440, 1310, 1180, 1080, 1035, 965, 931, 889, 845 , and $770 \mathrm{~cm}^{-1}$ also correlate well to its literature data $[73,74]$. .This result demonstrated the efficacy of polymerizing pyrrole by electropolymerization on an ITO substrate using an electrochemical cell.

Both PC and pyrrole are sensitive to oxidation, and so it was necessary to distill them before use. It should be noted that during electropolymerization, an oxidative layer formed on the ITO substrate causing it to turn brown and exhibit voltage overflow in the electrochemical cell due to the increased resistance on the ITO substrate. It is critical for the electrochemical cell containing TEAP and TBA electrolytes, that the electropolymerization reaction be run under anaerobic conditions to minimize oxidation of the electrode. The pTS electrolyte did not appear to be as sensitive to oxidation, and it may be possible to run the polymerization reaction under either aerobic or anaerobic conditions using this electrolyte. In addition, water can be used as the solvent instead of an organic.

Utilizing the electrochemical cell, the hydrogel can be UV photopolymerized with the cross-linker on the ITO substrate. In the pyrrole electrolyte solution, the force of the current generated between the electrodes may permit diffusion of the pyrrole monomer through the hydrogel on the ITO surface. Once the pyrrole monomer is in the hydrogel, electropolymerization of the pyrrole may generate a conductive polymer network of polypyrrole interpenetrating the hydrogel matrix. 


\section{CHAPTER V \\ DESIGN AND COMPOSITION OF THE POLYMER COMPOUND}

\subsection{Introduction}

The characteristics of the EAP and the hydrogel contribute towards the functionality of the polymer as an actuator and its biocompatibility, respectively. Polypyrrole (PPy) has unique conductive properties but it is very brittle and does not have the mechanical robustness of a hydrogel. It was evident that the hydrogel and the EAP each have mutually exclusive inherent properties that would benefit each other in its integration. Various EAPs and hydrogels may be synthesized and integrated together as a polymer compound using a combination of polymerization techniques (i.e. UVphotopolymerization, microwave assisted synthesis reaction, and electropolymerization). These targeted techniques minimize the use of organics and toxic materials in order to promote an improved biocompatible compound.

The actuation function of the electromechanical polymer compound may be dependent on its structure or physical composition. $\mathrm{Wu}$, et al and Madden, et al found the most responsive electromechanical actuators were synthesized as multiple thin layers of different materials to provide different elements for their actuator $[61,68]$. However, as it was mentioned in the introduction, the major disadvantage of composites is that delamination between the EAP and hydrogel typically occurs with repeated actuation. 
This leads to the argument that a supporting interpenetrating polymer network (IPN) may be preferred over composites because they are an interlaced network. The idea is that electromechanical actuators may be synthesized with the EAPs and hydrogels in different conformations and arrangements to achieve controlled and precise movement with maximum force.

\subsection{Design Approach of the Polymer Compound}

The design approach in the synthesis of the polymer compound is to develop a piece wise synthesis of the hydrogel and EAP so that the materials can be integrated into the desired compound. In the first step, a cross-linker was synthesized with the microwave assisted synthesis reaction. Following this, the cross-linkers were UV polymerized with the hydrogel on the indium tin oxide (ITO) substrate. The ITO substrate with the hydrogel was immersed in the electrochemical cell with the appropriate electrolyte solution and pyrrole concentration.

\subsection{Initial Compound Attempt Experimental Procedure}

A preliminary study was performed in an attempt to form a compound of the cross-linked poly(methacrylamide) (PMA) and PPy. This was the first step in the design of the polymer compound to incorporate the flexible and hydrophilic properties of the hydrogel with the conductive properties of PPy. The conductive element of the polymer compound needs to be developed first in our design before our secondary goal to establish mechanical actuation of polymer compound. 


\subsubsection{Experimental Procedure}

First, PMA cross-linked with PEG550DMA was synthesized on ITO glass by UV photopolymerization as outlined in Section 2.2.1 and Section 2.4.1. Utilizing Scheme 1, methacrylamide was mixed with DMPA and PEG550DMA at a weight percent of 5.0\% and $10 \%$, respectively. The monomer solution was then pipetted onto an ITO glass substrate fitted with a silicone mold to hold the solution. The ITO glass with the monomer solution was then exposed under UV for ten minutes at $365 \mathrm{~nm}$. The PMA thin film attached to the ITO substrate was then together placed into an electrolyte solution of 0.05M TEAP in PC to swell the hydrogel prior to electropolymerization.

Electropolymerization of a 0.1 the PPy solution was performed at $4^{\circ} \mathrm{C}$ in a $\mathrm{N}_{2}$ atmosphere and $0.2 \mathrm{~mA}$ in a TEAP electrolyte solution. The pyrrole monomer in the electrolyte solution was anticipated to diffuse into the swelled PMA hydrogel and PPy would electropolymerize within the thin hydrogel.

\subsubsection{Results and Discussion}

The electropolymerization of PPy within in the cross-linked PMA did not seem to show any significant polymerization of pyrrole within the hydrogel by physical appearance. The appearance of the PMA on the ITO substrate did not show any darkening as was expected by polymerization of PPy within the hydrogel. Conductivity measurements of the PMA post electropolymerization with pyrrole did not show any improvement in conductivity. To examine if any pyrrole polymerized within the PMA hydrogel a FTIR scan was done on the PMA hydrogel after the attempted electropolymerization of PPy within the hydrogel. To prepare the samples the hydrogel 
was pulverized into a power and a mull of the pulverized polymer powder with mineral oil was used in the FTIR analyzer. The mull was smeared on a $\mathrm{AgCl}$ plate and analyzed.

The presence of PPy in the PMA hydrogel can be identified in the sample by FTIR by identifying the notable IR absorption peaks for PPy within the spectrum of the polymer compound. Since, the polymer compound is comprised of several materials; the individual elements need to be identified. Figure 33 shows the unique FTIR spectra of PMA, PEGDMA, and mineral oil. The respective IR absorption peaks identified by arrows are:

- PMA; 3351, 3224, 3000, 1648, 1600, 1495, 1390, 1200, 1100, and $957 \mathrm{~cm}^{-1}$.

- PEGDMA; 2860, 1715, 1640, 1445, 1300, 1100, 940, 850, and $660 \mathrm{~cm}^{-1}$.

- mineral oil; $2920,2850,1470,1380$, and $720 \mathrm{~cm}^{-1}$ [75].

Figure 34 shows the IR spectra for PPy mulled with mineral oil from Section 4.4.2. With these unique IR absorption peaks, we can identify the presence or absence of PPY within the compound. For example in Figure 34, we can see both the presence of PPy and mineral oil in the IR spectra. However, based on the unique IR absorption peaks for mineral oil in Figure 33 c) we can identify the IR absorption peaks for PPy in Figure 34 to be $2720,2670,1547,1487,1440,1310,1180,1080,1035,965,931,889,845$, and 770 $\mathrm{cm}^{-1}$ as indicated by the arrows $[74,76]$.

Figure 35 shows the IR absorption spectra of the PMA hydrogel that was placed in the electrochemical cell for the attempt to electropolymerize PPy within it. Comparing the IR absorption peaks in Figure 33for PMA, PEGDMA, and mineral oil; and in 


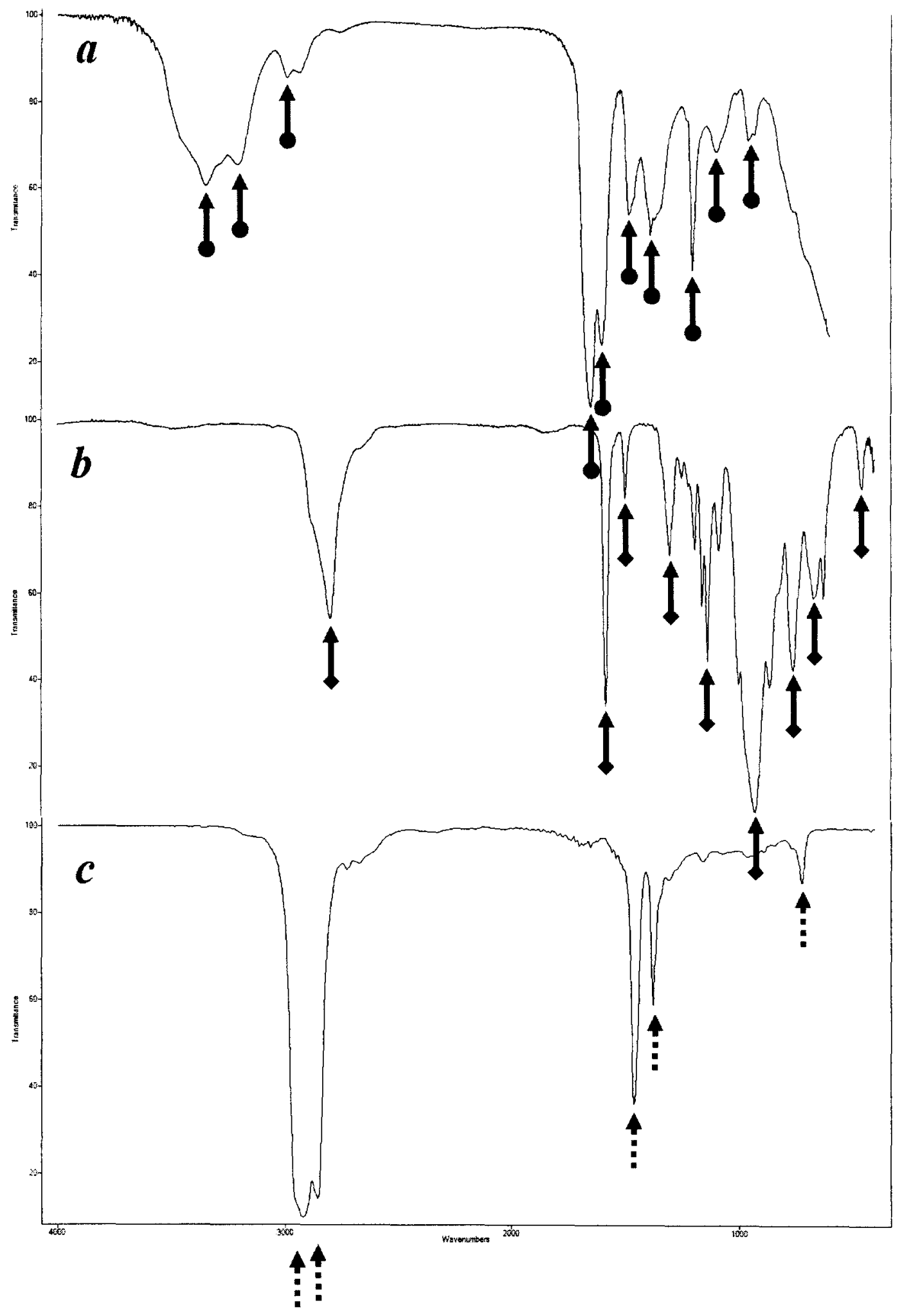

Figure 33. FTIR spectra of a) PMA, b) PEGDMA, and c) Mineral Oil. 


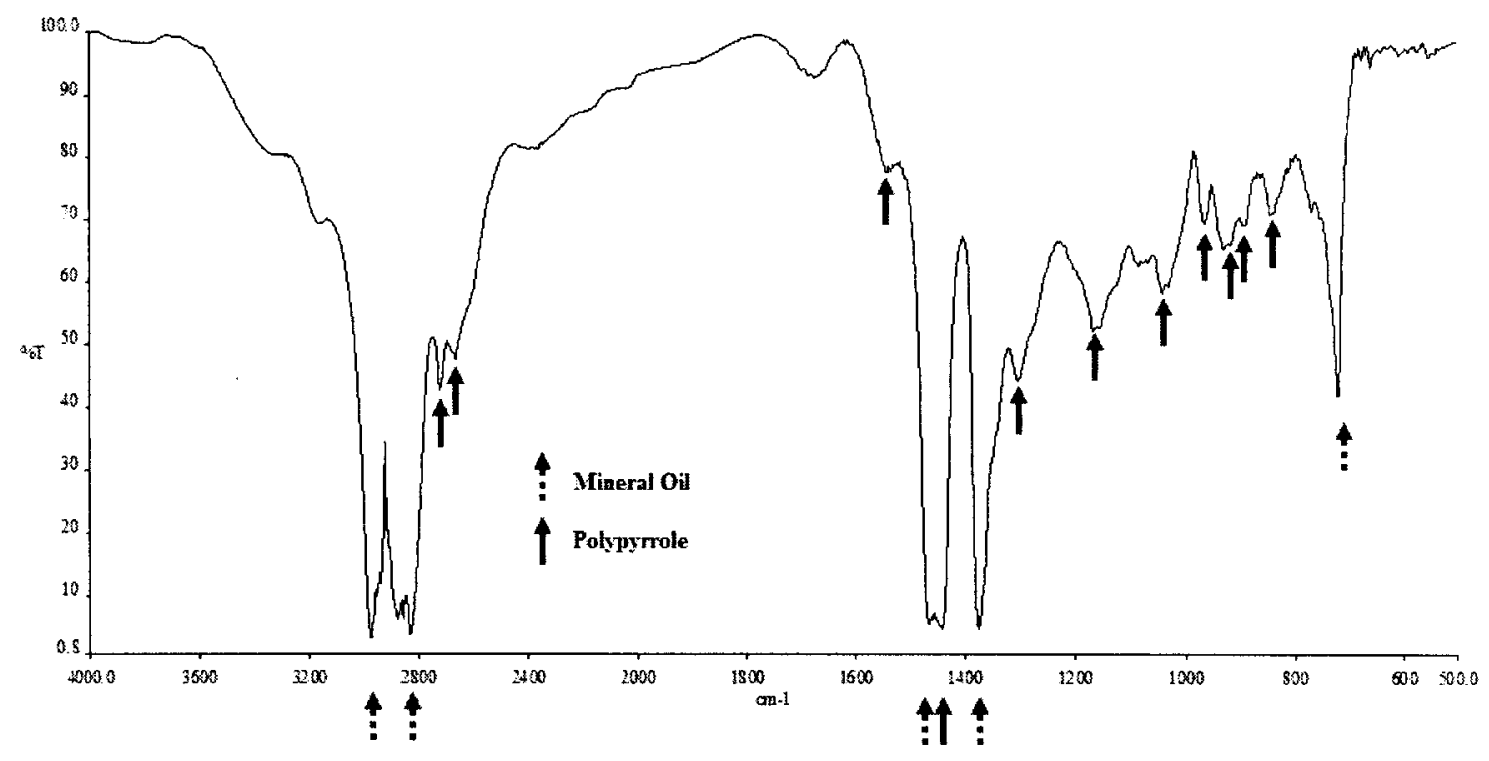

Figure 34. FTIR spectra of electropolymerized PPy.

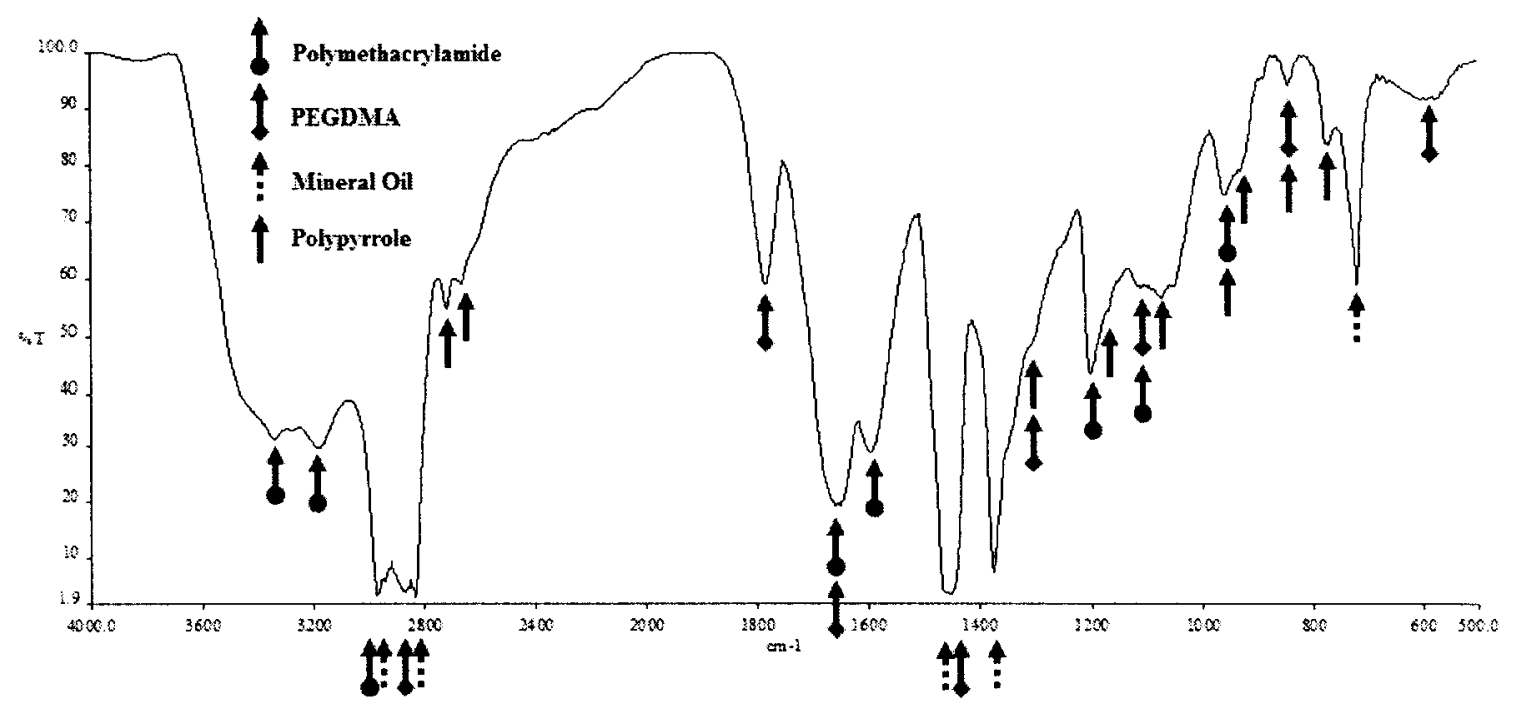

Figure 35. FTIR spectra of the electropolymerization of PPy within the photopolymerized PMA on ITO substrate. 
Figure 34 for PPy we can potentially filter the noteworthy IR absorption peaks for PPy in Figure 35. The presence of any PPy IR absorption peaks could identify the presence of any pyrrole that may have polymerized within the cross-linked PMA. If we compare the IR absorbance peaks for PPy in Figure 34 with the absorbance peaks for the sample in Figure 35 we can see that the IR absorbance peaks were:

- Polypyrrole (Figure 34)

$2720,2670,1547,1487,1440,1310,1180,1080,1035,965,931,889,845$, and $770 \mathrm{~cm}^{-1}$

- Polypyrrole electropolymerized in cross-linked PMA (Figure 35)

$$
\begin{aligned}
& 2720,2670, \quad 1310,1180, \quad 1035,965,931,889,845 \text {, and } \\
& 759 \mathrm{~cm}^{-1}
\end{aligned}
$$

The IR absorbance peaks in Figure 35 correlate well with the IR absorbance peaks for PPY. The presence of these IR absorbance peaks for PPY in Figure 35 indicated that some pyrrole may have polymerized within the PMA hydrogel. However, it is also plausible that the IR absorption peaks in Figure 35may be present for some pyrrole that may have diffused into the surface of the swollen PMA hydrogel. It is a possibility that a very minute amount of PPy polymerized within the PMA hydrogel. This can be supported by the fact that there was not any darkening or discoloration of the hydrogel as would be expected if PPy had polymerized within the PMA thin film. In addition, conductivity of the hydrogel did not increase. It was apparent that the hydrophobic pyrrole molecule may need to be modified to be hydrophilic in order to increase the ability of the pyrrole monomer to diffuse into the hydrogel. By increasing the diffusion 
of pyrrole into the hydrogel it may be possible to increase polymerization of pyrrole and, therefore, increase its conductivity.

There were issues with keeping the PMA hydrogel on the ITO substrate surface. While swelling the PMA hydrogel on the ITO substrate the hydrogel would delaminate from the ITO surface. Small amounts of tape had to be used to keep the hydrogel firmly on the ITO surface. It was also apparent that the surface of the ITO glass needed to be functionalized with hydrophilic groups to help hold the hydrogel to the surface of the ITO glass. To do this, the ITO substrate was modified with 3-aminopropyltrietheoxysilane (APTES) in order to aid in the adhesion of the hydrogel to the ITO during electropolymerization. Figure 36 shows an illustration of the APTES molecule.

The ITO substrate was sonicated in a detergent solution, water, acetone, and then ethanol for 15 minutes, respectively to clean the surface. The ITO substrate was then dried under a stream of nitrogen. The ITO was then immersed in an ethanol solution of $1 \%$ (v/v) APTES (3-aminopropyltriethoxysilane) for 30 minutes at $75^{\circ} \mathrm{C}$. It was then rinsed five times in ethanol with sonication and dried at $120^{\circ} \mathrm{C}$ for 3 hours to remove any loosely bound or physisorbed molecules. Figure 37 illustrates the silation of the ITO surface with APTES. This method replicated the protocol for treatment of the ITO substrate with APTES as reported by Wang et. al. [77,78]. 


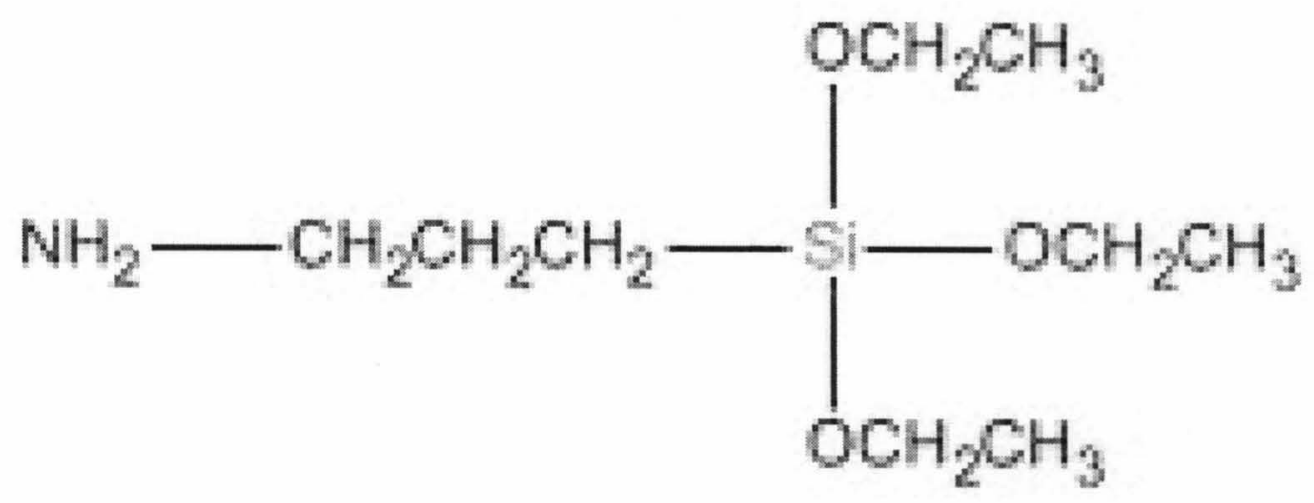

Figure 36. Molecular Structure of 3-Aminopropyltrioxysilane (APTES).

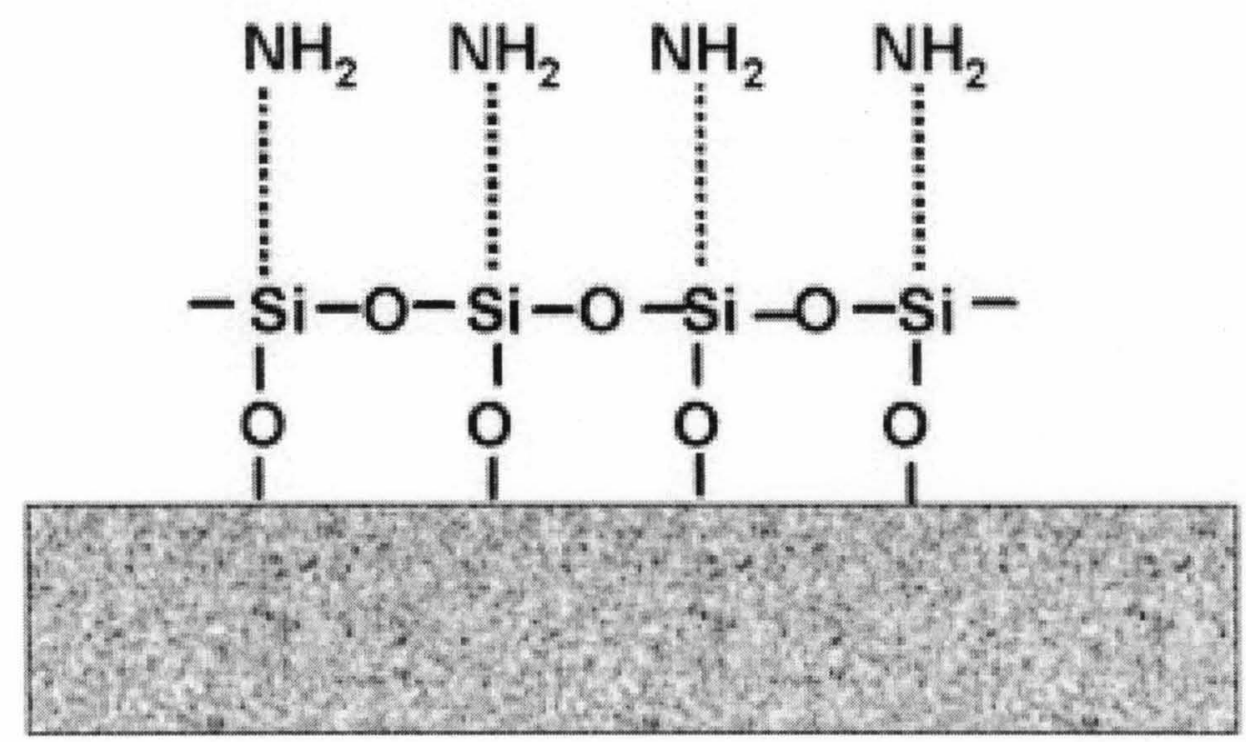

Figure 37. Illustration of silation of substrate surface with 3-aminopropyltrhioxysilane (APTES) 

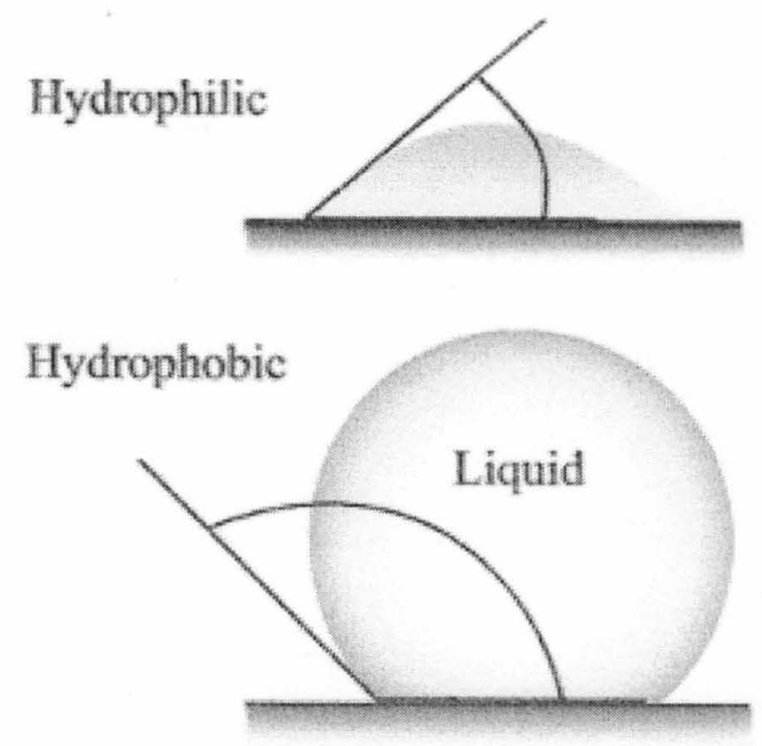

Figure 38. Illustration of the optical contact angle measurement on a liquid droplet.

The coverage of APTES on the ITO glass was determined by utilizing the optical contact angle measuring device (Figure 38). The optical contact measurements of water on the ITO glass were $91.8 \pm 4.6$ and $67.1 \pm 3.0$ for the untreated and treated ITO, respectively. The lower measurement of 67.1 on the APTES treated ITO glass demonstrated an increased hydrophilicity of the ITO surface.

\subsection{Modification of a Hydrophobic Pyrrole}

It was evident that the pyrrole monomer did not diffuse into the hydrogel due to its hydrophobic nature. To facilitate the diffusion of the pyrrole monomer into the hydrogel matrix, it needs to be modified with a hydrophilic group. However, the modification to the pyrrole monomer must be on the $\beta$ ( 3 or 4 ) position of the aromatic ring as shown in Figure 39. The controlled synthesis was very difficult and there is very 

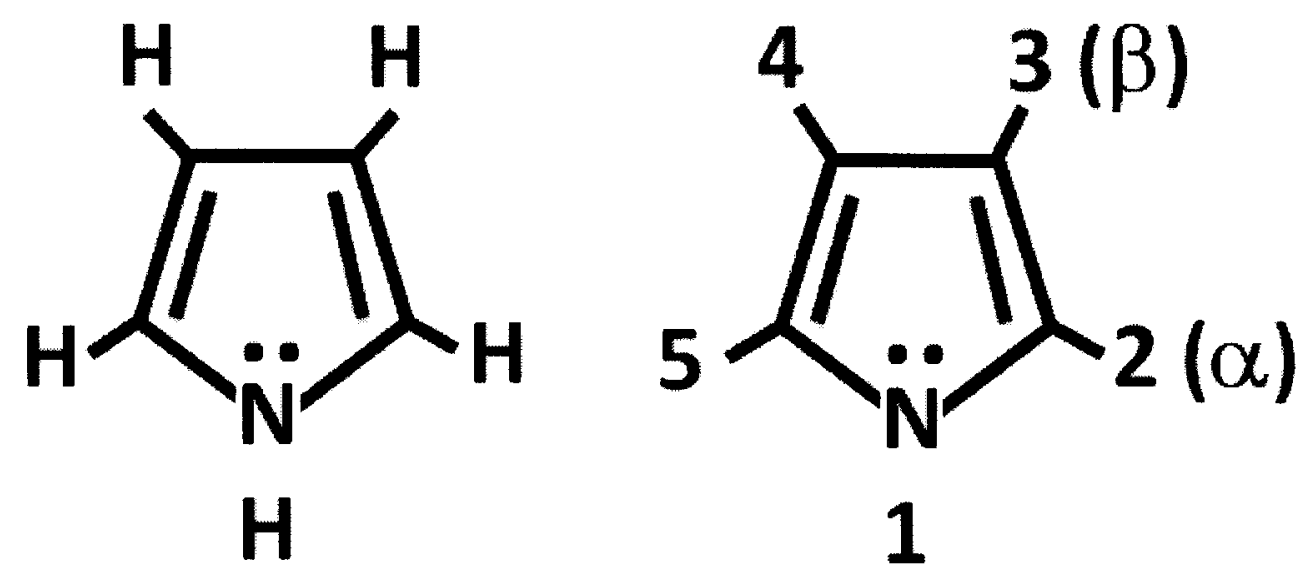

Figure 39. Pyrrole Molecule.

little literature on the subject of modifying the pyrrole monomer on the $\beta$ position in making the aromatic molecule hydrophilic. The following synthesis techniques employed in the modification of the pyrrole molecule were highly experimental and required a lot of control. The purpose of the following synthesis techniques were to minimize the affect a substituent may have on the delocalized electrons of the nitrogen, which provides the conduction pathway along the polypyrrole chain. This may be prevented by protecting the nitrogen molecule and the unbound pair of electrons with a protective group during the synthesis process for a hydrophilic pyrrole molecule.

\subsubsection{Protecting Groups}

The function of a protective group is to allow a chemical reaction to be carried out at one reactive site on a multifunctional compound while the other reactive sites are temporarily blocked. The protective group should fulfill the basic requirements of 
providing good yield to a protected substrate, it should be stable under the projected reactions, and it must be selectively removed in good yield of the substrate with easy separation [79]. The seven tactical consideration of an effective protecting group are:

- It should be cheap and readily available

- The protecting group should be easily and efficiently introduced

- It should be easy to characterize and avoid complications as the creation of new stereogenic centers.

- It should be stable to a wide range of reaction conditions

- It should be stable to a wide range of techniques for separation, purification.

- It should be removed selectively and efficiently under highly specific conditions.

- The by-products of the deprotection should be easily separated from the substrate [80].

Sulfonamides are amongst the most stable of nitrogen protecting groups. The powerful electron-withdrawing effect of the arylsulfonyl group reduces the high susceptibility of the pyrrole nucleus to electrophilic attack and oxidation. Sulfonamide derivatives of weakly basic amines such as pyrroles can be easily cleaved by simple basic hydrolysis. Replacement of the $\mathrm{N}-\mathrm{H}$ group in the pyrrole by an arylsulfonyl group allows the lithiation, nucleophilic aromatic substitution and subsequent reaction to the sulfoxide of an electrophilic aromatic substitution, of the heterocyclic nucleus.

In the present case the nitrogen nucleophile substitutes the chlorine of the electrophilic benzenesulfonyl chloride by the mechanism illustrated in Figure 40. Following the protection of the nitrogen, modification of the pyrrole monomer can 
proceed so that the modification occurs in the $\beta$ position of the pyrrole ring due in part to the steric protection of the $\alpha$ position by the arylsulfonyl group.
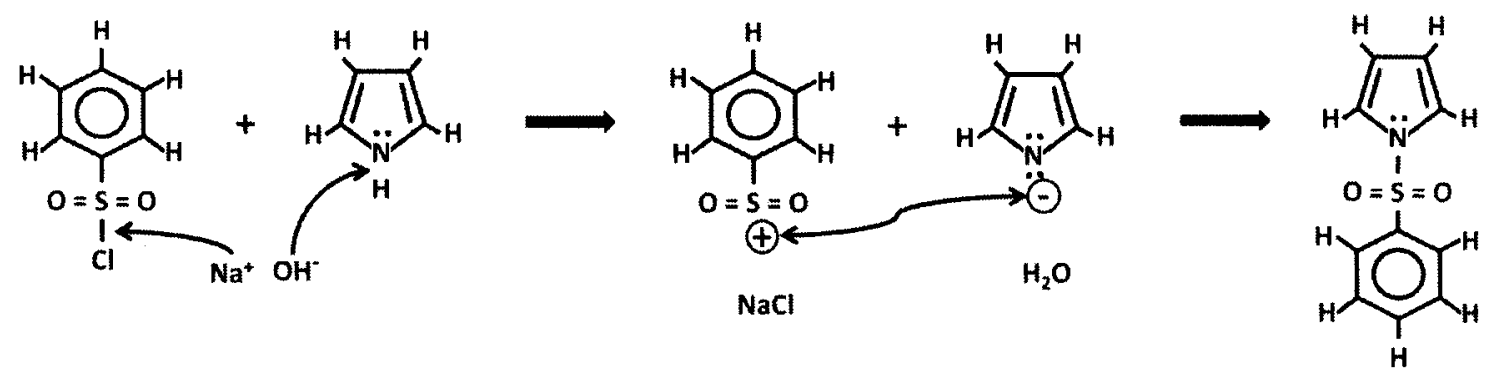

Figure 40. Mechanism of Benzenesulfonyl Protection of Pyrrole monomer.

\subsubsection{Experimental Procedure for the protection of pyrrole}

To a well stirred mixture of $\mathrm{NaOH}$ and water, pyrrole, methylene chloride $\left(\mathrm{CH}_{2} \mathrm{Cl}_{2}\right)$, and tetrabutylammonium hydrogen sulfate was added to a solution of benzenesulfonyl chloride and $\mathrm{CH}_{2} \mathrm{Cl}_{2}$ at a rate that allowed the mixture to be refluxed gently. External cooling was provided to control the exothermic reaction. After stirring overnight, water was added to the mixture and the organics were extracted with chloroform $\left(\mathrm{CHCl}_{3}\right)$. The organic layers were washed with water and dried with magnesium sulfate. After the water was evaporated, methanol was added to the residue and stirred. The methanol residue was suction filtered and the filtrate was evaporate a second time and filtered. 
5.4.1.2 Results of 1-(Phenylsulfonyl)pyrrole formation

Figure 41illustrates the protected pyrrole molecule 1-(Phenylsulfonyl)pyrrole and the correlation of the $\mathrm{H}$ atoms on the molecule to the ${ }^{1} \mathrm{H}$ NMR of the product from the reaction mechanism in Figure 42. The ${ }^{1}$ H NMR data in Figure 42shows that the formation of the protected pyrrole was successful. The yield from this reaction was $60 \pm 2 \%$.

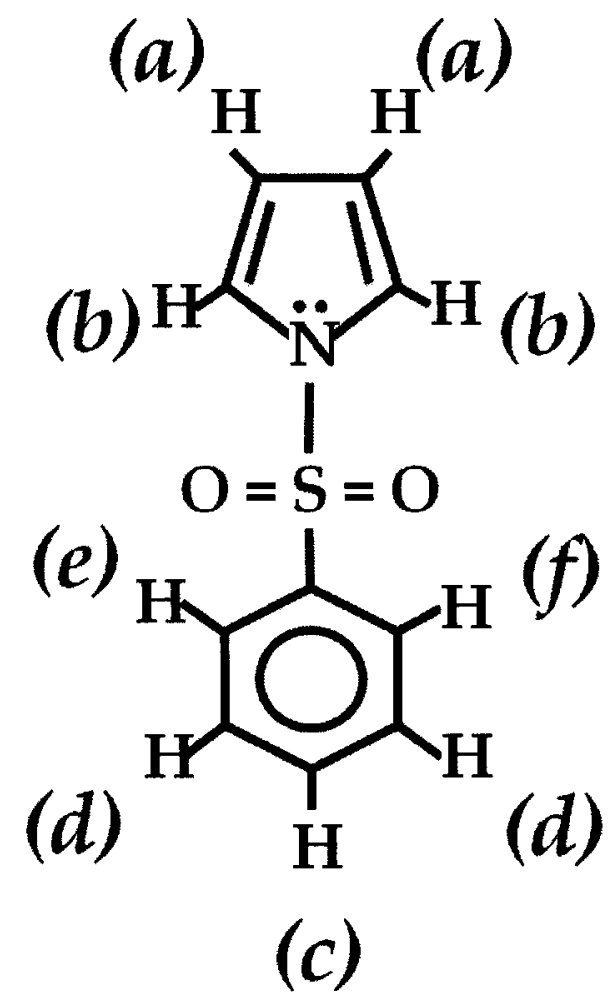

Figure 41. Illustration of protected pyrrole molecule, 1-(Phenylsulfonyl) pyrrole molecule with $\mathrm{H}$ atoms identified for the ${ }^{1} \mathrm{H}$ NMR spectra. 


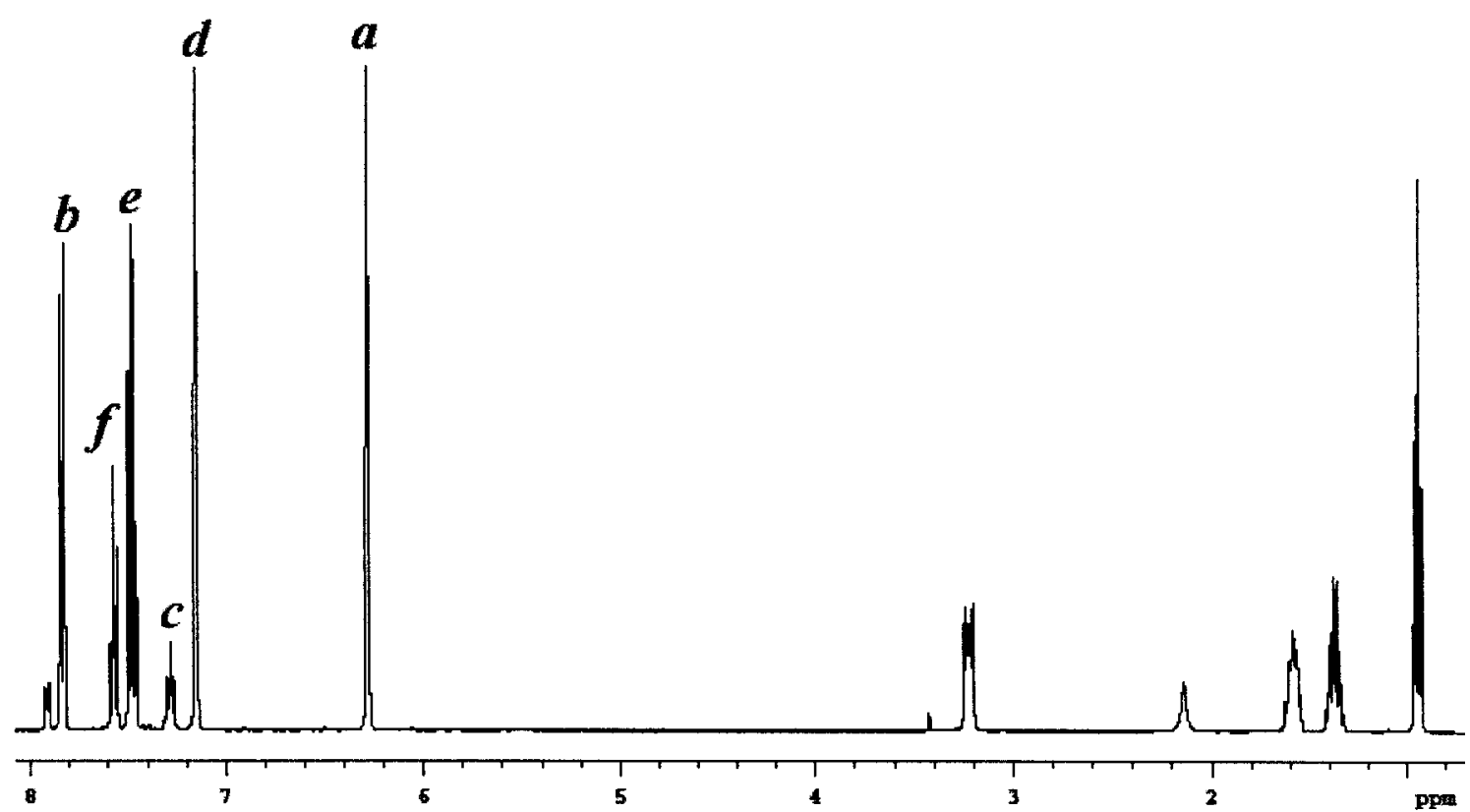

Figure 42. ${ }^{1} \mathrm{H}$ NMR $\left(\mathrm{CDCl}_{3}\right)$ spectra of 1-(Phenylsulfonyl)pyrrole - (b) 7.83, 2H; (f) 7.55, $\mathrm{H}$; (e) 7.48, H; (d) 7.28, 2H; (c) 7.28, H; (a) 6.28, 2H.

\subsubsection{Modification of Pyrrole Monomer}

In the succeeding step, the Friedel-Crafts acylation mechanism with $\mathrm{AlCl}_{3}$ was used to acylate the 3 position of the protected pyrrole ring as illustrated Figure 43 . The complete acylation mechanism and the activation of the $\mathrm{AlCl}_{3}$ are shown in Appendix D. Friedel-Crafts Alkylation Mechanism. Following the Friedel-Crafts acylation, a Clemmensen reduction was performed on the protected acylpyrrole with amalgamated zinc to give a 3-( $\omega$-haloalkyl)pyrrole. The complete Clemmensen reduction reaction is shown in Appendix E. Clemmensen Reduction Mechanism Following the Clemmensen reduction, the intermediate alkylpyrrole was sulfonated to convert it to a sulfonated 
alkylapyrrole. The sulfonation reaction mechanism is shown in Appendix F. Sulfonation Reaction with $\mathrm{SO}_{3}$
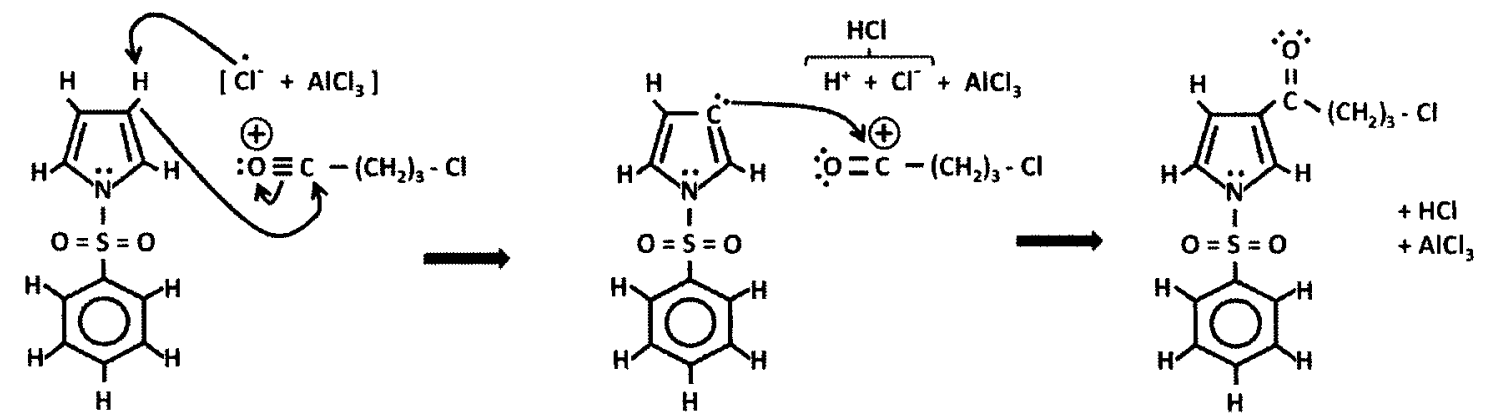

Figure 43. Alkylation Reaction Mechansim of 1-(Phenylsulfonyl)Pyrrole.

and the carboxylation reaction mechanism in Appendix G. Carboxylation Reaction with $\mathrm{CO}_{3}$ The sulfonated alkylpyrrole was then deprotected to remove the protective group from the nitrogen atom within the aromatic pyrrole. The deprotection mechanism is shown in Appendix H. Deprotection Mechanism

\subsubsection{Experimental Procedure Friedel-Crafts Acylation of 1- (Phenylsulfonyl)Pyrrole}

A mixture of 4-chlorobutyryl chloride in $\mathrm{CH}_{2} \mathrm{Cl}_{2}$ was added over 20 minutes to a stirred mixture powder of $\mathrm{AlCl}_{3}$ in $\mathrm{CH}_{2} \mathrm{Cl}_{2}$ with a cooling bath to control the exothermic reaction. After continued stirring for 45 minutes a clear solution resulted and the 1-[1(phenylsulfonyl)pyrrole in $\mathrm{CH}_{2} \mathrm{Cl}_{2}$ was added over a cooling bath. After stirring one hour the organic solution is poured over water and shaken. The organic layer is separated and 
extracted from the water with $\mathrm{CHCl}_{3}$. The organic layer was washed with a $\mathrm{NaOH}$ solution and water successively. Methanol was then added to the turbid organic layer to form two clear layers. The lower layer was dried with magnesium sulfate and evaporated.

5.4.2.2 Results of 1-[1-(Phenylsulfonyl)-3pyrrolyl]-4-chloro-1-butanone formation

Figure 44 illustrates the 1-[1-(Phenylsulfonyl)-3pyrrolyl]-4-chloro-1-butanone molecule and the correlation of the $\mathrm{H}$ atoms on the molecule to the ${ }^{1} \mathrm{H}$ NMR of the product from this reaction in Figure 45. Figure 45 shows that the formation of the 1-[1(Phenylsulfonyl)-3pyrrolyl]-4-chloro-1-butanone intermediate molecule was successful. The yield from this reaction was $80 \pm 13 \%$.

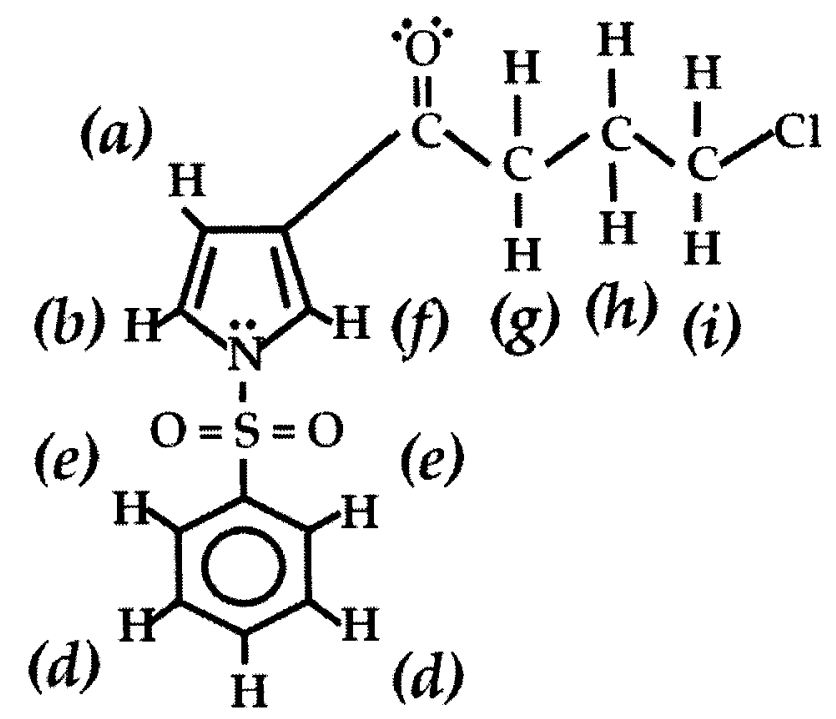

Figure 44. Illustration of 1-[1-(Phenylsulfonyl)-3-pyrrolyl]-4-chloro-1-butanone molecule with $\mathrm{H}$ atoms identified for the ${ }^{1} \mathrm{H}$ NMR spectra. 


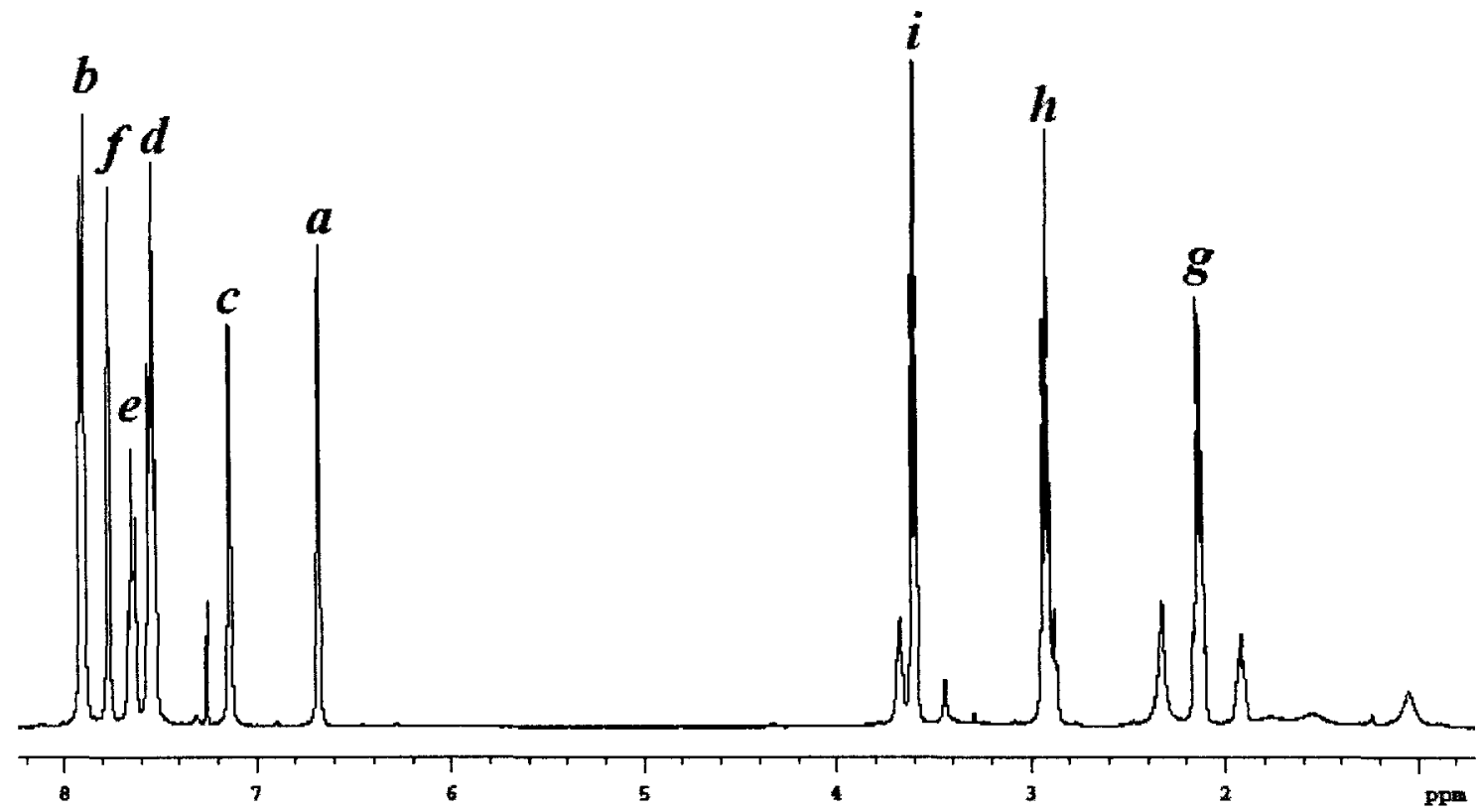

Figure 45. ${ }^{1} \mathrm{H}$ NMR $\left(\mathrm{CDCl}_{3}\right)$ spectra of 1-[1-(Phenylsulfonyl)-3-pyrrolyl]-4-chloro-1butanone - (b) 7.9, $\mathrm{H}$; (f) 7.77, $\mathrm{H}$; (e) 7.65,2 H; (d) 7.75, 2H; (c) 7.14, H; (a) 6.68, 2H; (i) $3.6,2 \mathrm{H} ;$ (h) 2.92, $2 \mathrm{H} ;$ (g) 2.13, $2 \mathrm{H}$.

5.4.2.3 Experimental Procedure for the Clemmensen Reduction of 1-[1-(Phenylsulfonyl)3pyrrolyl]-4-chloro-1-butanone

1-[1-(Phenylsulfonyl)-3pyrrolyl]-4-chloro-1-butanone was dissolved in toluene and water and added to amalgamated zinc. Amalgamated zinc was prepared as described by Anderson et. al. [81] with fine zinc powder, $\mathrm{HgCl}_{2}$, and concentrated $\mathrm{HCl}$.

Concentrated $\mathrm{HCl}$ was added to the mixture and heated for 2.5 hours with good stirring and gentle reflux so that foaming was not too excessive during the reaction. During the heating period when the foaming stopped, smaller portions of concentrated $\mathrm{HCl}$ were successively added three times. After cooling the mixture, the organic layer was 
separated and the water layer was extracted with toluene. The combined organic layers were washed with water and dried with magnesium sulfate. The organics were dried to yield a dark oil.

\subsubsection{Results for 1-[1-(Phenylsulfonyl)-3pyrrolyl]-4-chlorobutane formation}

Figure 46 illustrates the 1-[1-(Phenylsulfonyl)-3pyrrolyl]-4-chlorobutane molecule and the correlation of the $\mathrm{H}$ atoms on the molecule to the ${ }^{1} \mathrm{H}$ NMR of the product from this reaction in Figure 47. Figure 47 shows that the formation of the 1-[1(Phenylsulfonyl)-3pyrrolyl]-4-chlorobutane intermediate molecule was successful. The yield from this reaction was $81 \pm 24 \%$.

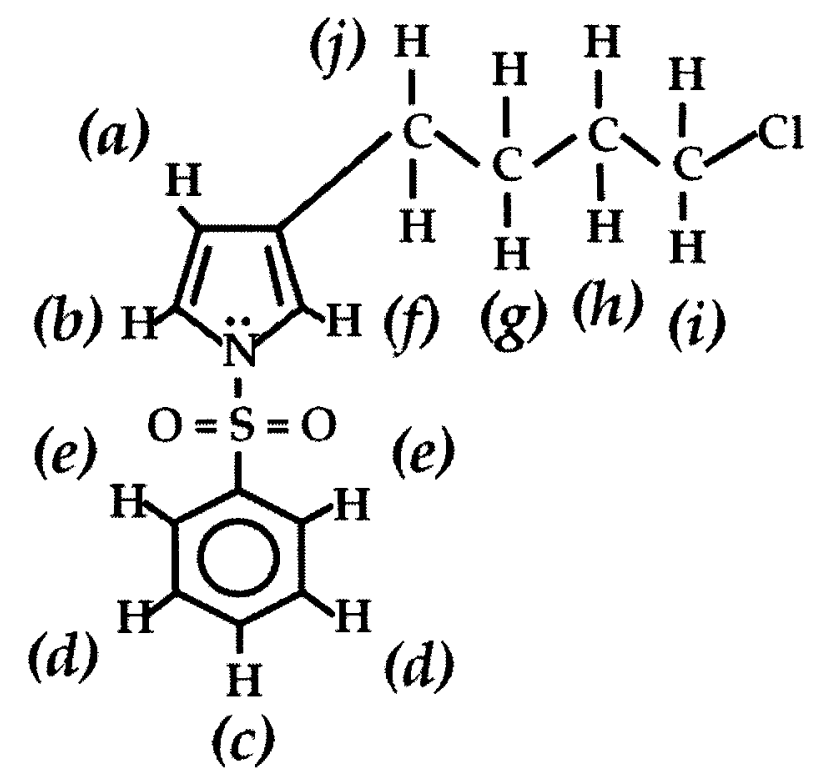

Figure 46. Illustration of 1-[1-(Phenylsulfonyl)-3-pyrrolyl]-4-chlorobutane molecule with $\mathrm{H}$ atoms identified for the ${ }^{\mathrm{l}} \mathrm{H}$ NMR spectra. 


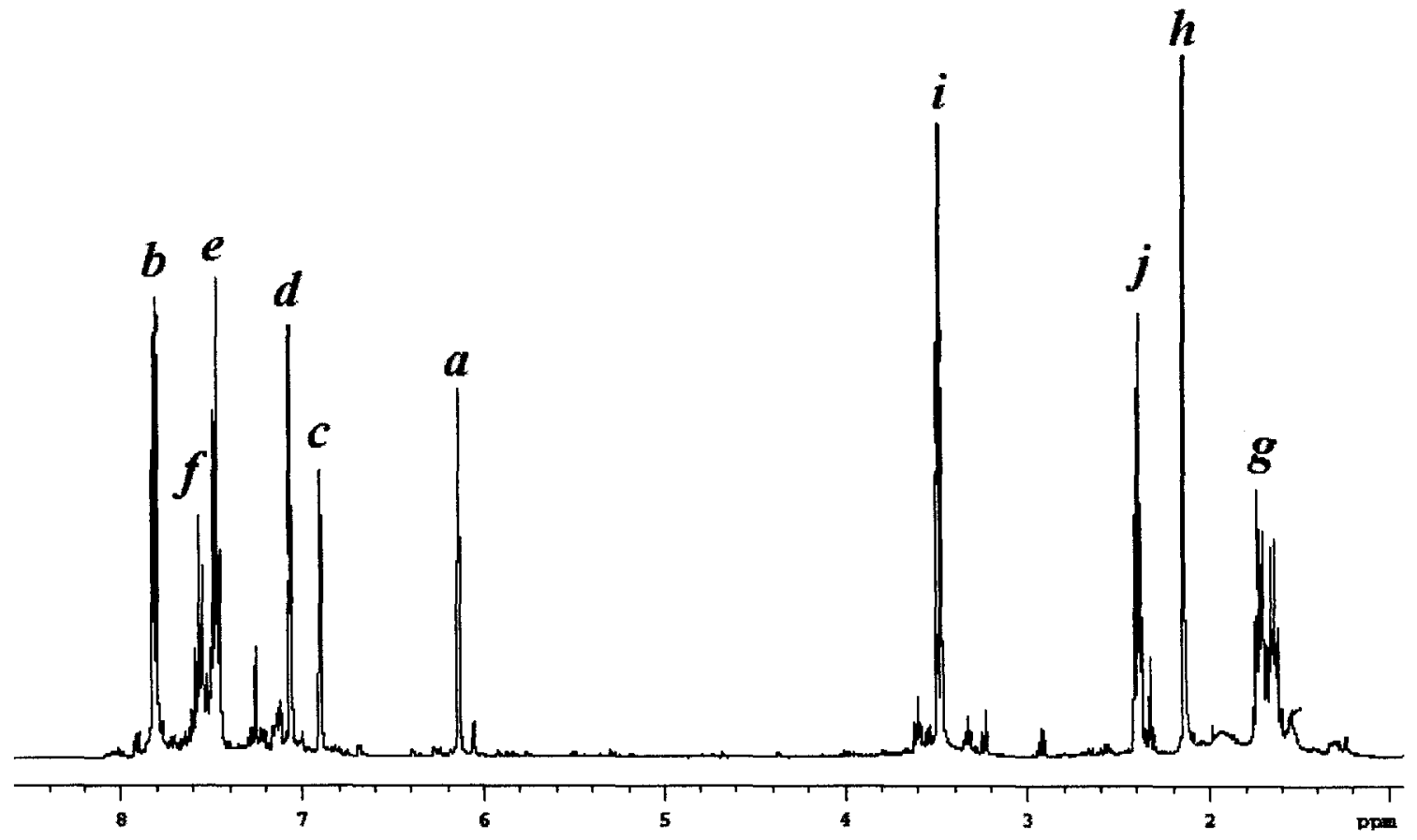

Figure 47. ${ }^{1} \mathrm{H}$ NMR $\left(\mathrm{CDCl}_{3}\right)$ spectra of 1-[1-(Phenylsulfonyl)-3-pyrrolyl]-4chlorobutane - (b) 7.80, H; (f) 7.55, H; (e) 7.48, 2H; (d) 7.07, 2H; (c) 6.90, H; (a) 6.28, H; (i) $3.40,2 \mathrm{H}$; (j) 2.40,2H, (h) 2.14, 2H, (g) 1.72, H, 1.65, H.

5.4.2.5 Experimental Procedure for the Sulfonation and deprotection of 1-[1(Phenylsulfonyl)-3pyrrolyl]-4-chlorobutane

A mixture of 1-[1-(Phenylsulfonyl)-3pyrrolyl]-4-chlorobutane, water, and ethanol, sodium sulfite, tetrabutylammonium bromide, and sodium iodide were heated under reflux for 18 hours for the sulfonation reaction. To this mixture $\mathrm{NaOH}$ was added and reacted for 1.5 hours under reflux for the deprotection reaction and then brine was added. The mixture was cooled and extracted twice with $\mathrm{CHCl}_{3}$. The organic layer was washed with water and the combined aqueous layers were evaporated. The residue was boiled 
with water and allowed to cool to room temperature. The resulting paste was suction filtered and washed with brine. The wet solid was boiled with $96 \%$ ethanol and $2-$ chloroethanol to neutralize the suspension. The hot suspension was filtered hot, and the remaining solids were boiled with $90 \%$ ethanol and filtered hot. The filtrates were combined and evaporated. The residue was boiled again with $90 \%$ ethanol, filtered hot, and evaporated. This residue was boiled with $96 \%$ ethanol and some water to give a solution. After cooling this solution to room temperature, it was filtered to remove any impurities. The filtrate was then cooled to $-15^{\circ} \mathrm{C}$ and the crystalline product was suction filtered and washed with $96 \%$ ethanol and acetone. The filtrates were evaporated again and the residue was recrystallized with $96 \%$ ethanol and some water. After cooling to $15^{\circ} \mathrm{C}$ again, the crystalline product was suction filtered to obtain more products.

\subsubsection{Results of 4-(3-Pyrrolyl)butanesulfonic Acid, Sodium Salt formation}

Figure 48 illustrates the 4-(3-Pyrrolyl)butanesulfonic Acid molecule and the correlation of the $\mathrm{H}$ atoms on the molecule to the ${ }^{1} \mathrm{H}$ NMR of the product from this reaction in Figure 49. Figure 49 shows that the formation of the 4-(3Pyrrolyl)butanesulfonic Acid, Sodium Salt hydrophilic pyrrole molecule was successful. However, the yield from this reaction was only $8.4 \pm 5 \%$. 


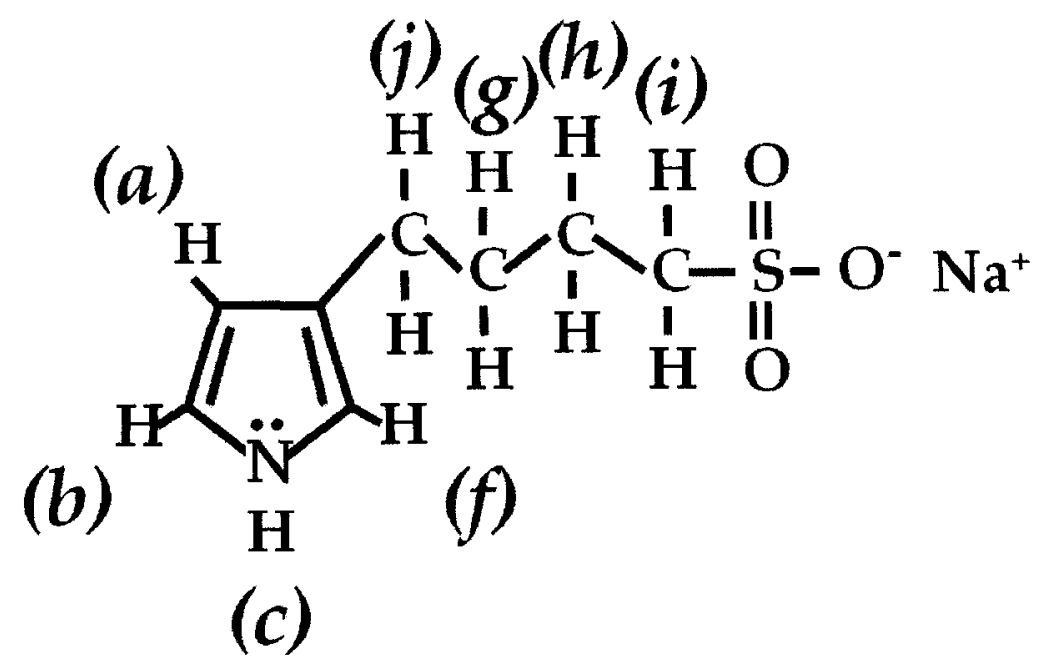

Figure 48. Illustration of 4-(3-Pyrrolyl)butane sulfonic Acid, Sodium Slat molecule with $\mathrm{H}$ atoms identified for the ${ }^{1} \mathrm{H}$ NMR spectra.

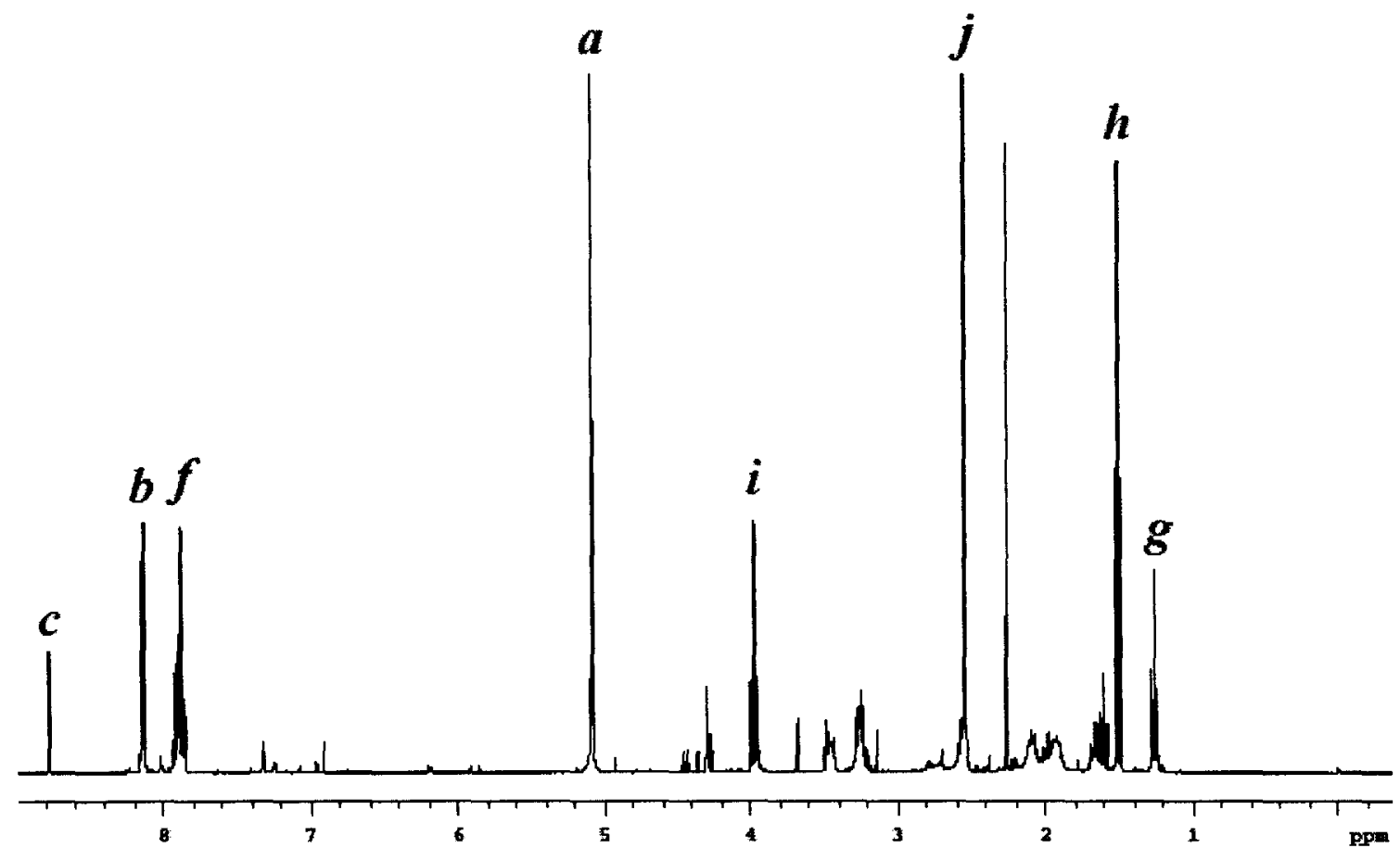

Figure 49. ${ }^{1} \mathrm{H}$ NMR $\left(\mathrm{D}_{2} \mathrm{O}\right)$ spectra of 4-(3-Pyrrolyl)butanesulfonic Acid, Sodium Salt (c) $8.78, \mathrm{H}$; (b) $8.13, \mathrm{H}$; (f) 7.88, H; (a) $5.08, \mathrm{H}$; (i) 3.98, 2H; (j) 2.54, 2H, (h) 1.50, 2H, (g) $1.26,2 \mathrm{H}$. 


\subsubsection{Modification of the Pyrrole Monomer with a Carboxylic Ion}

Following the above synthesis technique cited by Havingia et. al [82] to modify the hydrophobic pyrrole with a sulfonated salt, it was desired to try and modify the pyrrole with a different hydrophilic group. Sodium carbonate was chosen in attempts to modify the hydrophobic pyrrole with a carboxylic ion. The potential outcome of a different hydrophilic group may provide an increased hydrophilicity and better diffusion of the pyrrole monomer into the hydrogel. This may allow for increased concentrations of pyrrole in the hydrogel during the electropolymerization of pyrrole in the electrochemical cell and thereby increase the conductivity of the polymer compound.

5.4.3.1 Experimental Procedure for the carboxylation of 1-[1-(Phenylsulfonyl)-3pyrrolyl]-4-chlorobutane

The same procedures as the sulfonation and deprotection of 1-[1(Phenylsulfonyl)-3-pyrrolyl]-4-chlorobutane was followed for the carboxylation of 1-[1(Phenylsulfonyl)-3-pyrrolyl]-4-chlorobutane except for the fact that sodium carbonate was used in place of sodium sulfite.

\subsubsection{Results of 4-(3-Pyrrolyl)butanecarboxylic Acid, Sodium Salt formation}

Figure 50 illustrates the 4-(3-Pyrrolyl)butanecarboxylic Acid molecule and the correlation of the $\mathrm{H}$ atoms on the molecule to the ${ }^{1} \mathrm{H}$ NMR of the product from this reaction in Figure 51. Figure 51shows that the formation of the 4-(3Pyrrolyl)butanecarboxylic Acid, Sodium Salt hydrophilic pyrrole molecule was 


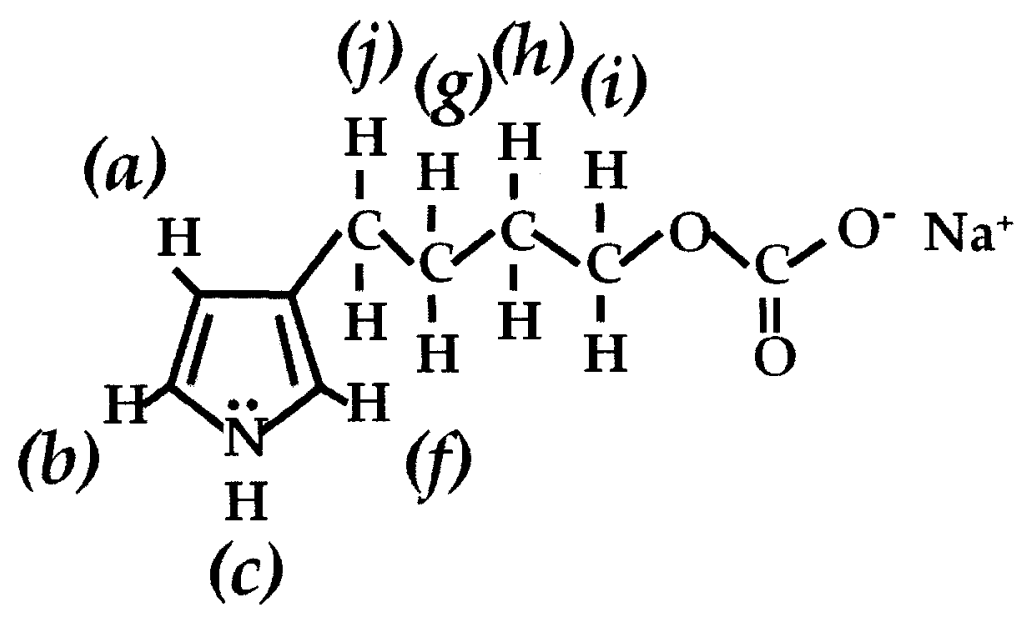

Figure 50. Illustration of 4-(3-Pyrrolyl)butane carboxylic Acid, Sodium Slat molecule with $\mathrm{H}$ atoms identified for the ${ }^{1} \mathrm{H}$ NMR spectra.

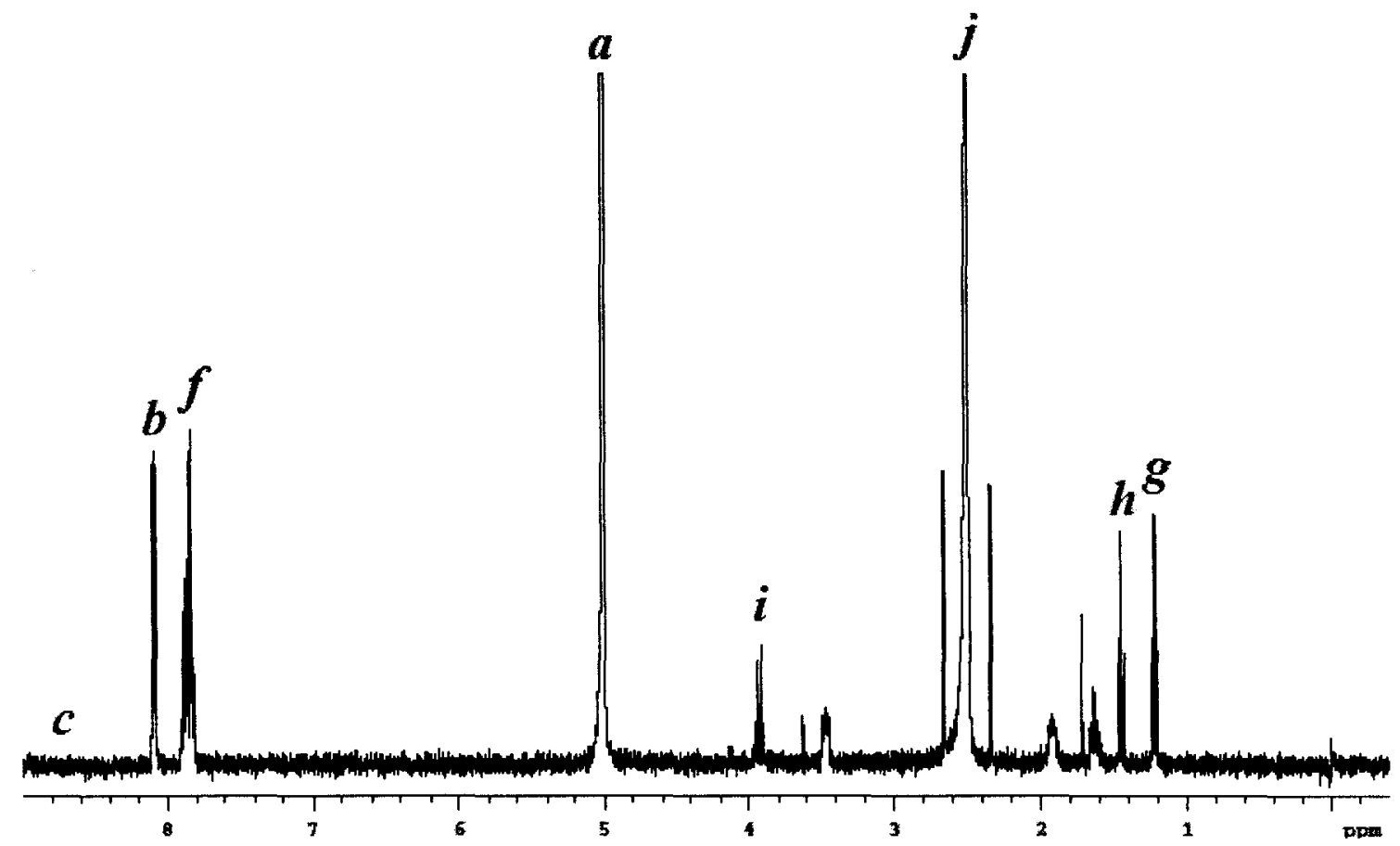

Figure 51. ${ }^{1} \mathrm{H}$ NMR $\left(\mathrm{D}_{2} \mathrm{O}\right)$ spectra of 4-(3-Pyrrolyl)butanecarboxylic Acid, Sodium Salt

- (c) $8.78, \mathrm{H}$; (b) 8.09, H; (f) 7.84, H; (a) 5.00, H; (i) 3.92, 2H; (j) 2.50, 2H, (h) 1.46, 2H,

(g) $1.22,2 \mathrm{H}$. 
successful. Figure 52 shows the presence of all 8 carbon atoms in the 4-(3-

Pyrrolyl)butane carboxylic Acid molecule. However, the yield from this reaction was only $6.3 \%$.

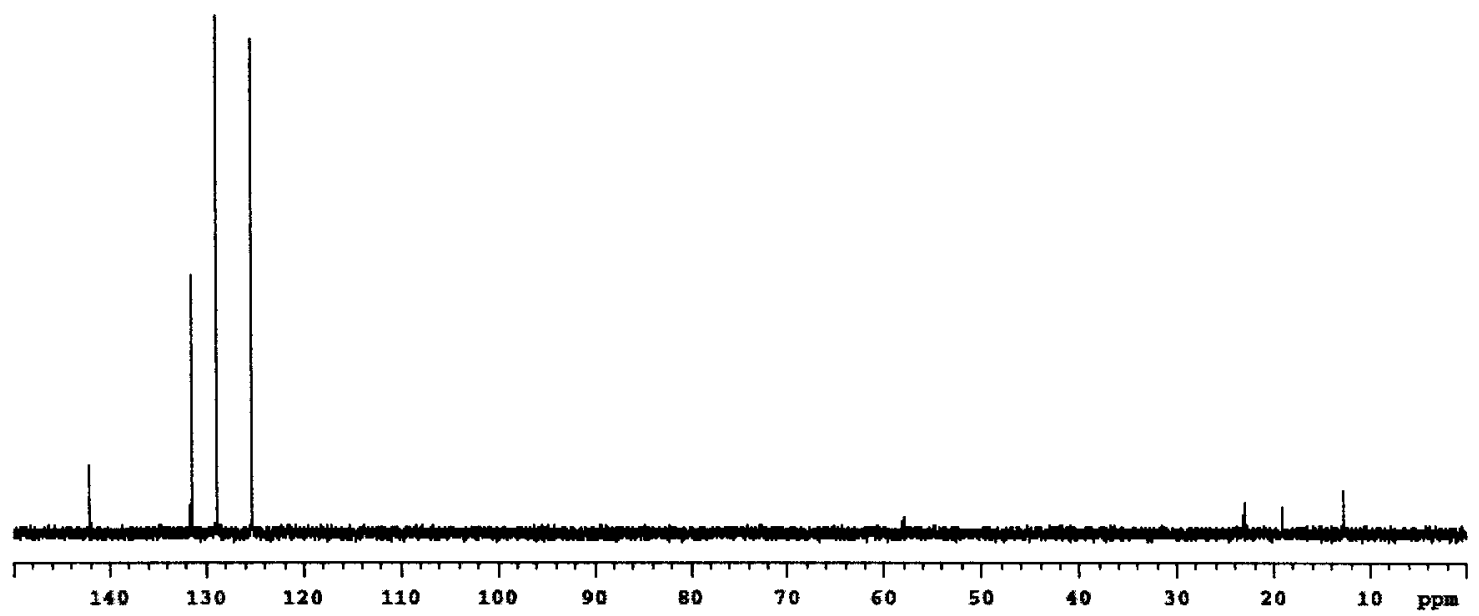

Figure 52. ${ }^{13} \mathrm{C}$ NMR $\left(\mathrm{D}_{2} \mathrm{O}\right)$ spectra of 4-(3-Pyrrolyl)butanecarboxylic Acid, Sodium Salt $-142.2,131.5,128.9,125.3,58.0,23.0,19.1,12.8$

\subsubsection{Discussion}

The chemistry involved in the modifying a specific carbon within the aromatic pyrrole monomer, namely the $\beta$ position as was illustrated in Figure 19 in Chapter 3 had to be precisely controlled and was difficult. This experimental synthesis method for the targeted modification of pyrrole was highly experimental in that very little had been cited in literature about modification of pyrrole at the $\beta$ position. Protecting the pyrrole monomer at the nitrogen atom and utilizing the synthesis scheme above, it was possible 
to modify the hydrophobic pyrrole monomer with both a sulfonate and carbonate hydrophilic group. Havinga et. al. reported a 40\% yield of 4-(3-Pyrrolyl)butanesulfonic Acid, Sodium Salt. The yield of both the 4-(3-Pyrrolyl)butanesulfonic Acid and 4-(3Pyrrolyl)butanecarboxylic Acid, Sodium Salts was very low and may be improved by modifying the final purification step. If the yield can be improved for the modified hydrophilic pyrrole, it will make the next step of the development of the polymer compound much easier. The modified hydrophilic pyrrole monomer will be used in the electrochemical cell to hopefully improve the polymerization of pyrrole into the PMA hydrogel.

\subsection{Polymer Compound Formation with Hydrophilic Pyrrole}

In the initial attempt at forming the PMA-PPY polymer compound it was found that the pyrrole may have had decreased diffusion into the cross-linked PMA because the pyrrole molecule was hydrophobic. Therefore in an attempt to increase the potential diffusion of pyrrole into the hydrogel and increase the concentration of polypyrrole within the hydrogel, the pyrrole monomer was modified with a hydrophilic group. This hydrophilic pyrrole was utilized for the PMA-PPY polymer compound formulation.

\subsubsection{Experimental Scheme}

The same set up for the electrochemical cell was used as described in Chapter 4. However, the ITO substrate was functionalized with APTES as described in Section 5.3.2 of Chapter 5. In addition, no supplementary electrolytes needed to be utilized because 
the hydrophilic pyrrole salt also acted as a conduction salt. Also, water could be used in place of an organic solvent because the hydrophilic pyrrole is water soluble.

\subsubsection{Result \& Discussion}

In Figure 53 of Section 5.3.2, the IR absorbance spectra for PPy in mineral oil that was presented in Figure 32 of Section 4.4.2 is revisited. The IR absorbance spectra from Figure 35 of Section 5.3.2 for the initial electropolymerization of PPy in the cross-linked PMA is also reexamined in Figure 54. In both Figure 53 and Figure 54, it was noted in Section 5.3.2 that the IR absorbance peaks for PPy, which were indicated by arrows, coincided relatively well:

- Unmodified PPy (Hydrophilic pyrrole, Figure 53);

$2720,2670,1547,1487,1440,1310,1180,1035,965,931,889,845$, and $759 \mathrm{~cm}^{-1}$

- Unmodified PPy in cross-linked PMA (Figure 54);

$2720,2670, \quad 1310,1180,1080,965,931,889,845$, and $770 \mathrm{~cm}^{-1}$

In Figure 54, the \% $\mathrm{T}$ at the IR absorbance peaks for PPy decreased slightly from the IR absorbance spectra of PMA in Figure 33a). This indicated that there could have been some hydrophilic pyrrole which may have polymerized within or one the PMA thin film. 


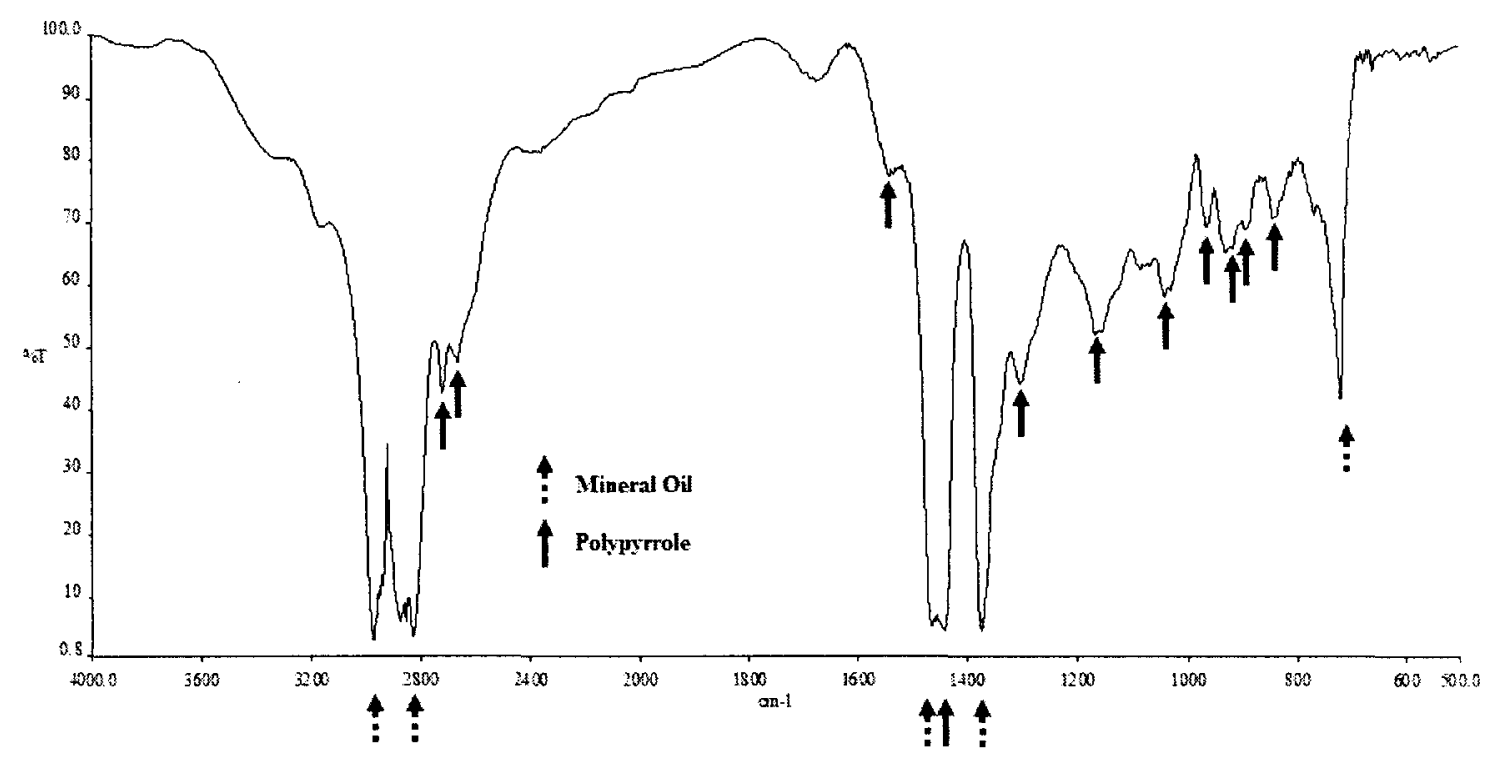

Figure 53. FTIR spectra of electropolymerized PPy in mineral oil.

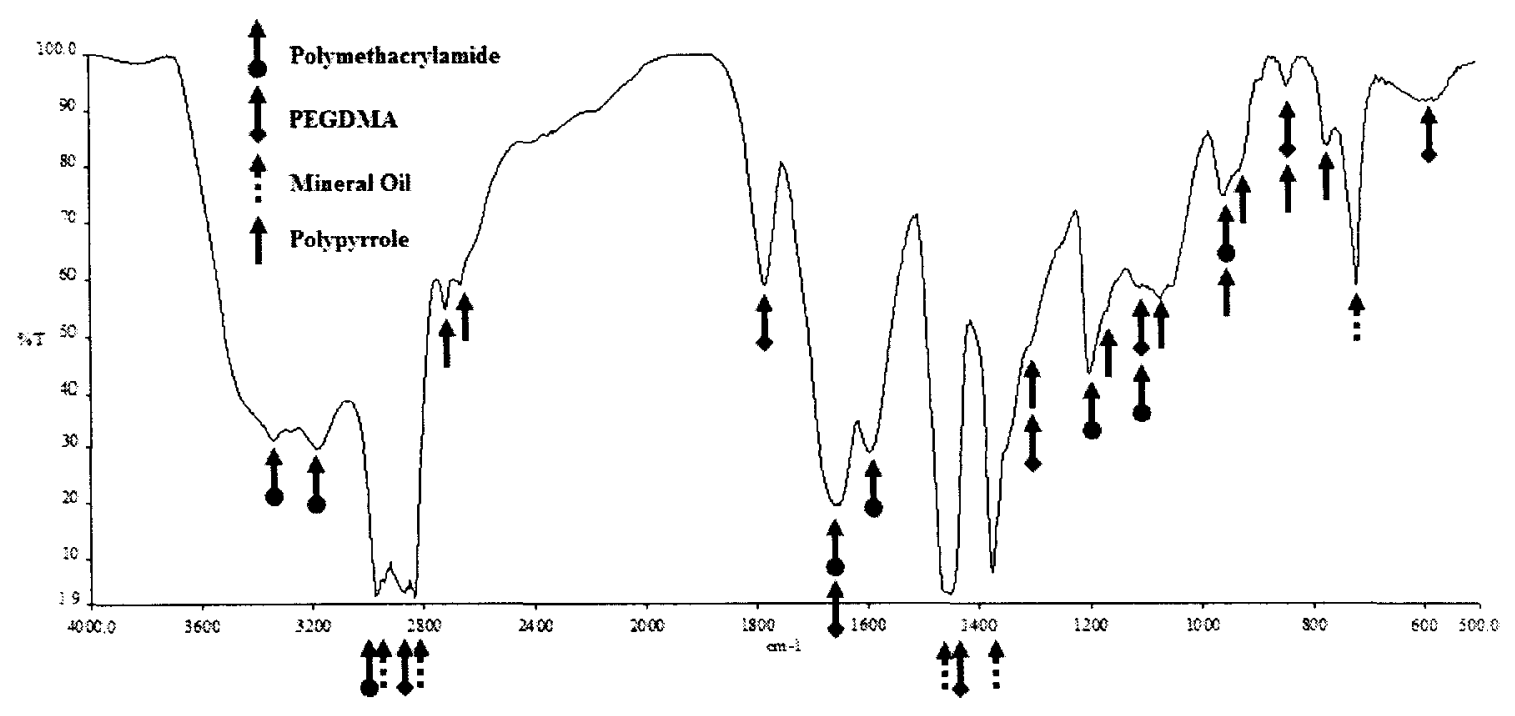

Figure 54. FTIR spectra of initial attempt at electropolymerizing PPy within PMA photopolymerized on ITO 


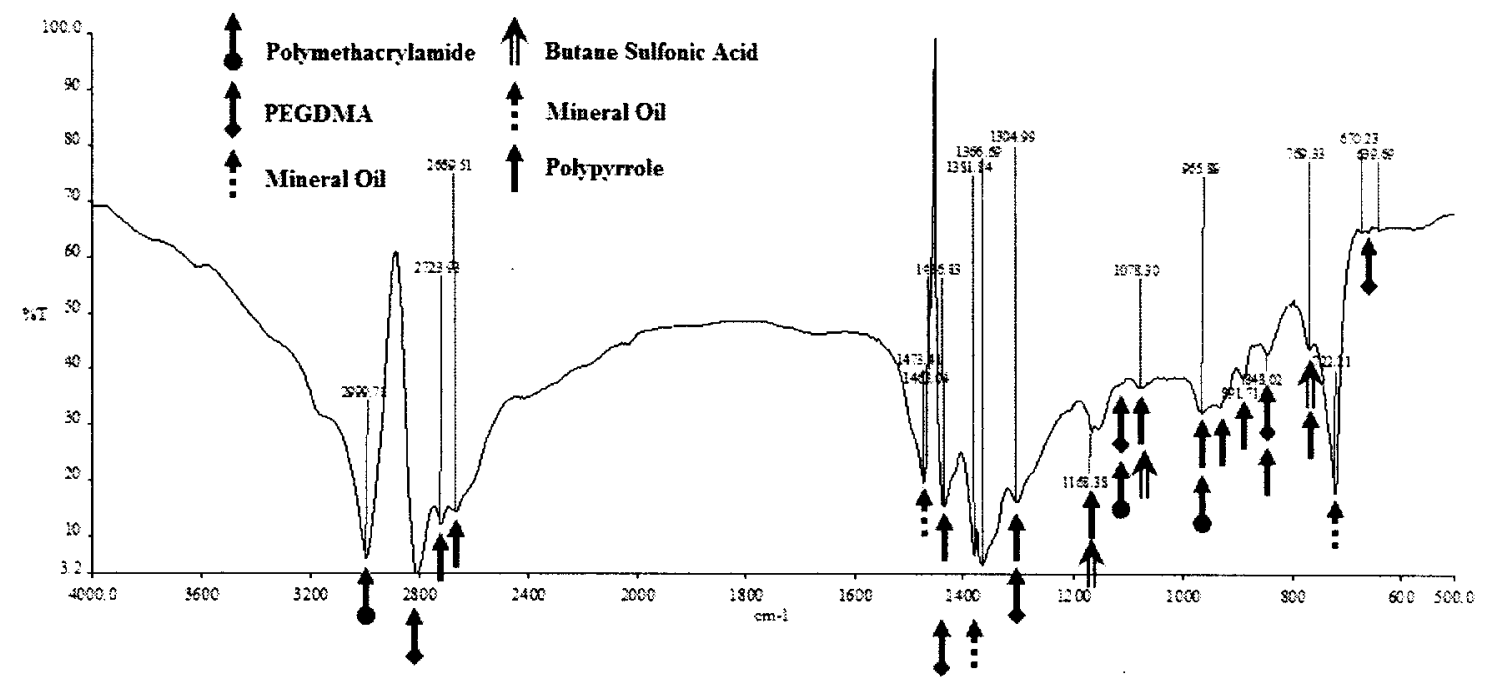

Figure 55. FTIR spectra of PMA electropolymerization with 4-(3-

Pyrrolyl)butanesulfonic Acid, Sodium Salt in water.

Next we compared the FTIR spectra of the cross-linked PMA electropolymerized with the unmodified hydrophobic pyrrole (Figure 54) and the modified hydrophilic pyrrole (Figure 55). The IR absorption peaks for PPy, as indicated by the arrows for PPy in the FTIR spectra, within the cross-linked PMA were:

- PPy, Unmodified hydrophobic pyrrole (Figure 54)

$2720,2670, \quad 1310,1180,1080,965,931,889,845$, and $770 \mathrm{~cm}^{-1}$

- PPy, Modified hydrophilic pyrrole salt, (Figure 55) $2720,2670,1440,1310,1180,1080,965,931,889,845$, and $770 \mathrm{~cm}^{-1}$.

The IR absorption peaks for PPy in correlate well in both Figure 54 and Figure 55. However, the IR absorbance peaks for PPy in Figure 55 showed decreased \%T as compared to the IR absorbance peaks for PPy in Figure 54. The increased IR absorption 
peaks for PPy indicated increased presence and potential polymerization of PPy within the cross-linked PMA. The IR absorbance peaks for the butane sulfonic acid side group was:

- Hydrophilic side group, butane sulfonic acid side group $1200,1185,1067$, and near $731 \mathrm{~cm}^{-1}$.

These IR absorbance peaks in the FTIR spectra are also identified but by different arrows in Figure 55. The presence of these IR absorbance peaks indicated the polymerization of the 4-(3-Pyrrolyl)butanesulfonic acid ion within the cross-linked PMA. This was an improvement in the inclusion of PPy into the hydrogel from the unmodified pyrrole monomer in Figure 54.

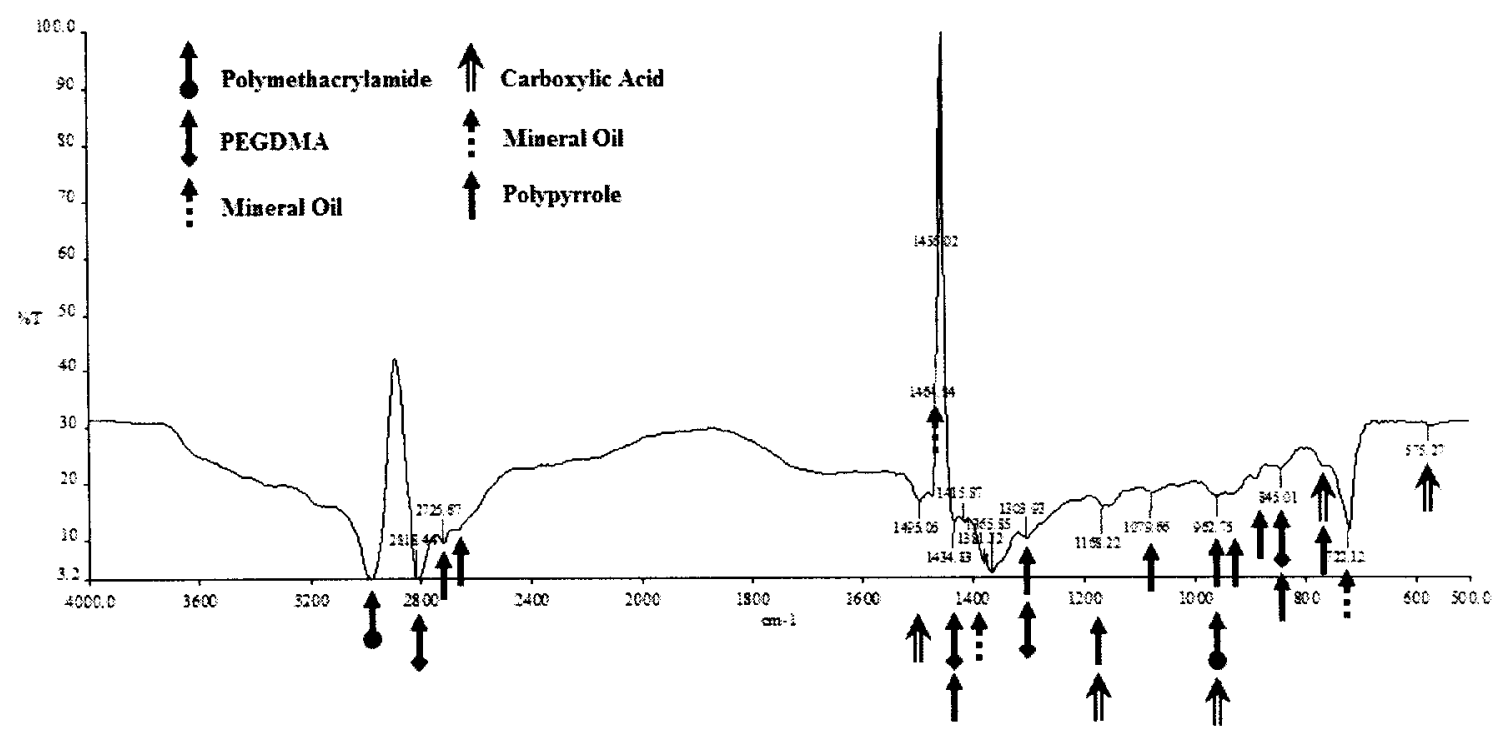

Figure 56. FTIR spectra of PMA electropolymerization with 4-(3-

Pyrrolyl)butanecarboxylic Acid, Sodium Salt in water. 
The pyrrole monomer was modified with a different hydrophilic side group. In the second case a carboxylic ion was modified to the pyrrole monomer to form 4-(3Pyrrolyl)butanecarboxylic Acid, Sodium Salt. This hydrophilic pyrrole salt was also electropolymerized with the cross-linked PMA. We again compared the FTIR spectra of the cross-linked PMA electropolymerized with the unmodified hydrophobic pyrrole (Figure 54), the modified hydrophilic sulfonated pyrrole (Figure 55), and the modified hydrophilic carboxylated pyrrole (Figure 56). The IR absorption peaks for PPy, as indicated by the arrows for PPy in the FTIR spectra, within the cross-linked PMA were:

- PPy, Unmodified hydrophobic pyrrole (Figure 54) $2720,2670, \quad 1310,1180,1080,965,931,889,845$, and $770 \mathrm{~cm}^{-1}$

- PPy, Modified hydrophilic sulfonated pyrrole salt, (Figure 55) $2720,2670,1440,1310,1180,1080,965,931,889,845$, and $770 \mathrm{~cm}^{-1}$.

- PPy, Modified hydrophilic carboxylated pyrrole salt, (Figure 56) $2720,2670,1440,1310,1180,1080,965,931,889,845$, and $770 \mathrm{~cm}^{-1}$

These IR absorbance peaks for PPy in Figure 56 correlated well with IR absorbance peaks for PPy in Figure 54 and Figure 55. The IR absorbance peaks for PPy in Figure 56 again showed decreased $\% \mathrm{~T}$ at the absorption peaks for PPy when compared to the IR absorbance peaks for PPy in Figure 54. This again indicated that the modified hydrophilic pyrrole potentially aided in increased diffusion of the pyrrole into the PMA hydrogel and potentially increased the presence and concentration of PPy in the polymer compound. In fact, the IR absorbance for PPy in the PMA with the carboxylated side group (Figure 56) is higher than that observed for sulfonated side group (Figure 55). The 
inclusion of PPy into the cross-linked PMA hydrogel may be higher with the 4-(3Pyrrolyl)butanecarboxylic pyrrole ion in comparison to the 4-(3-Pyrrolyl)butanesulfuric pyrrole ion.

Comparison of the IR absorbance peaks for the butane sulfonic acid side group (Figure 55) with that of the carboxylic acid side group (Figure 56) in the FTIR spectra as indicated in both figures were:

- Hydrophilic side group, butane sulfonic acid side group (Figure 55) $1200,1185,1067$, and near $731 \mathrm{~cm}^{-1}$.

- Hydrophilic side group, butane carboxylic acid side group (Figure 56) $1495,1177,1067$, and near $731 \mathrm{~cm}^{-1}$.

The IR absorbance peaks for the butane carboxylic acid side group are mostly similar to that of the butane sulfonic acid side group, but slightly different at $1495 \mathrm{~cm}^{-1}$ and 1200 $\mathrm{cm}^{-1}$ for the carboxylic and sulfonic side group, respectively. This subtle difference indicates the polymerization of the 4-(3-Pyrrolyl)butanecarboxylic acid ion within the cross-linked PMA

By modifying the pyrrole monomer with a different hydrophilic group, carboxylic ion, it may be possible to increase the hydrophilic nature of the hydrophobic pyrrole and increase the diffusion of the pyrrole into the PMA hydrogel and therefore increase its electropolymerization within it. Modifying the pyrrole had allowed the decreased use of organic solvents in the electrochemical cell. In addition additional electrolytes were not needed due to the conductive properties of the hydrophilic pyrrole salt. However, the conductivity of the PMA hydrogel with the modified PPy did not result in increased 
conductivity of the polymer compound. The modified pyrrole may increase inclusion of PPy within the hydrogel but higher concentrations of the hydrophilic pyrrole salt may not be high enough to see enhanced conductivity. While the hydrophilic side group aids the diffusion of pyrrole into the hydrogel, the size of the side group may hinder the polymerization of PPy. Increasing the pyrrole concentration may help with this problem or we may need to look at a smaller side group. 


\section{CHAPTER VI}

\section{CONCLUSIONS}

The motivation behind this dissertation was to develop a biocompatible material that was conductive can could potentially support the muscle like movement and performance of cardiac muscles. The development of this polymer compound required a novel and methodical approach. It first required the study and understanding of each of the exclusive elements and then the integration of the elements into a single compound. The compound developed in this dissertation utilizes the swelling and flexible characteristics matrix of a hydrogel, poly(methacrylamide), and the conductive and electroactive properties of the conductive polymer, polypyrrole. The methods employed throughout the development this novel polymer compound required the selection of synthesis and polymerization techniques that minimized the use of organics, which could decrease the biocompatibility of the compound.

Results discovered with the study of the hydrogels revealed that UV polymerization was an effective method for the polymerization of a variety of hydrogels. Examinations showed that methacrylamide displayed the desirable soft gel-like properties sought for in the development of the polymer compound. However, a PEGDMA crosslinker was necessary to improve the structural integrity of the poly(methacrylamide) hydrogel. The microwave assisted synthesis reaction technique was utilized to reduce the 
use of organics in the synthesis of various molecular weight PEGDMA cross-linkers. The rigidity of the hydrogel was directly influenced by the weight percent of the photoinitiator, DMPA. Increased weight percent of DMPA during photopolymerization increased the rigidity of the hydrogel. It was also demonstrated that the flexibility of the poly(methacrylamide) was directly related to the molecular weight of the PEGDMA cross-linker. Larger molecular weight PEGDMA cross-linkers provided increased flexibility in the hydrogel due to increased spacing between polymer chains.

As expected the hydrogels were not conductive. An attempt to increase the conductivity of the hydrogel by doping it with gold nanoparticles at $0.2 \mathrm{wt} \%$ demonstrated no observable increase in electrical conductivity. It was evident in the SEM images that the gold nanoparticle concentration was too low to and its dispersion throughout the hydrogel was not adequate enough to allow for surface plasmon resonance of the gold nanoparticles to enhance electrical conductivity. The hydrogel doped with 0.2 $w t \%$ gold appeared to be more brittle than the plain hydrogel. This indicated possible alterations in the physical properties of the hydrogel such as the swelling ratio. It was unlikely that utilizing gold nanoparticles in the hydrogel could provide the necessary electrical conductivity while maintaining the desirable physical properties.

Conductive polymers were examined to provide enhancements within the hydrogel. Polypyrrole was selected as the most favorable of the various conductive polymers because of its electro active properties, biocompatibility, and it is the most broadly studied among conductive polymers. An electropolymerization process, which again minimized the use of organics, was designed to polymerize pyrrole on ITO substrate under a $\mathrm{N}_{2}$ atmosphere to minimize oxidation of the electrodes. Polypyrrole 
films were electropolymerized on the ITO substrate and showed similar levels of conductivity from the three electrolyte solutions examined, TEAP, TBA, and pTS. However, the polypyrrole films were brittle and not responsive to the application of an electrical current.

An initial attempt to electropolymerize polypyrrole into a PEGDMA cross-linked poly(methacrylamide) photopolymerized on the ITO substrate was not successful. It was projected that the force of the current generated between the electrodes may permit diffusion of the pyrrole monomer through the hydrogel polymerized on the ITO surface. FTIR results indicated that there was presence of some polypyrrole within the hydrogel but it was not significant enough to enhance the conductivity of the material. It became apparent that the hydrophobic nature of pyrrole had to be modified with a hydrophilic group to help facilitate increased diffusion into the hydrogel. It also became apparent that the surface of the ITO glass needed to be functionalized with hydrophilic groups using APTES to help hold the hydrogel to the surface of the ITO glass.

The chemistry involved in the modifying the pyrrole monomer required a very novel and highly experimental methodology. Modification of pyrrole on the $\beta$ position or 3 carbon of the aromatic ring is uncommon and there is very little literature cited. The synthesis of a hydrophilic pyrrole monomer required a very selective scheme which required the protection of the nitrogen in the aromatic ring of the pyrrole monomer. The nitrogen in pyrrole carries two delocalized electrons which are mainly responsible for the conductive properties of polypyrrole. Utilizing a synthesis scheme to modify pyrrole with a hydrophilic group at the $\beta$ position, NMR data confirm that two hydrophilic 
monomers were synthesized, one with a sulfonate group [ 4-(3-Pyrrolyl)butanesulfonic Acid, Sodium Salt] and another with a carbonate group[4-(3-Pyrrolyl)butanecarboxylic Acid, Sodium Salt]. The synthesis of these hydrophilic pyrrole monomers were very difficult and required multiple intermediate steps that resulted in very low yields of only $8.4 \pm 5 \%$ and $6.3 \%$ for the sulfonate and carbonate pyrroles, respectively. The synthesis and purification methods in the final steps may be amended to improve yield for the modified hydrophilic pyrrole.

The modified hydrophilic pyrrole monomers were then used in the scheme to synthesize the polymer compound with an interpenetrating network of poly(methacrylamide) and pyrrole. PMA cross-linked with PEGDMA was photo polymerized on an APTES modified ITO substrate. The hydrogel/ITO substrate was placed in an electrochemical cell with either the sulfonated or carbonated pyrrole in the electrolyte solution. Modification of the pyrrole eliminated use of organic solvents in the electrochemical cell because the hydrophilic pyrrole salts were water soluble. In addition, electrolytes were not needed due to the fact that the pyrrole salts is itself an electrolyte. FTIR results for the hydrogel electropolymerized with 4-(3-Pyrrolyl)butanesulfonic Acid, Sodium Salt and electropolymerized with 4-(3-Pyrrolyl)butanecarboxylic Acid, Sodium Salt both showed decrease in \%T of the IR absorbance peaks for PPy as compared to the IR peaks of the hydrogel electropolymerized with the unmodified pyrrole monomer. It should be noted that the IR absorbance peak for PPY in the 4-(3Pyrrolyl)butanecarboxylic Acid was higher than that observed for 4-(3Pyrrolyl)butanesulfonic Acid. This indicated that the diffusion of pyrrole into the hydrogel was increased by modifying pyrrole with hydrophilic groups. It may be 
possible that there is an increased concentration of polypyrrole electropolymerized within the hydrogel. However, the conductivity of the hydrogel with the modified hydrophilic pyrrole did not increase within the polymer compound. It may be necessary to increase the concentrations of the hydrophilic pyrrole salt within the electrochemical cell to increase both the diffusion and concentration of polypyrrole in the hydrogel.

A novel polymer compound was developed through a systematic approach of examining the components of multiple polymer compounds and then developing a method of integrating them into an interpenetrating network. The synthesis and polymerization techniques were discriminately chosen to minimize the use of organics in order to minimize any biocompatibility issues we may have with materials. It was demonstrated that various molecular weight PEG cross-linkers may be created through microwave assisted synthesis reactions. It was established that the poly(methacrylamide) hydrogels cross-linked with PEGDMA cross-linkers could be UV photopolymerized onto modified ITO substrates. The ITO substrates were modified with APTES to increase hydrophilic adhesion of the hydrogel. The diffusion of pyrrole into the hydrogel was improved by the synthesis of a modified pyrrole monomer with a hydrophilic group. This novel hydrophilic pyrrole salt is very unique in its structure due to the location of the hydrophilic group at the $\beta$ position as compared to the more common modification at the nitrogen or $\alpha$ position. Also, this hydrophilic pyrrole salt facilitated additional removal of organics and other chemicals from the development process by eliminating the necessity of both the organic solvent and electrolyte from the electrochemical cell. This interpenetrating network of polypyrrole polymerized within a hydrogel matrix provides a unique material which has a lower probability of delamination and a possibility for 
increased electrical conductivity. The fundamental concept for the development of biocompatible and conductive polymer compound has been established for the future application toward the development of an artificial cardiac muscle. 


\section{CHAPTER VII}

\section{FUTURE WORK}

The fundamental concepts for the development of biocompatible and conductive polymer compound have been established for the potential application toward an artificial cardiac muscle. The following are the immediate areas that will need attention for the future development of the hydrogel/polypyrrole polymer compound.

\subsection{Hydrogel Characterization and Improvements}

The ability of the hydrogel to swell is highly dependent on the degree of crosslinks. This may also affect the ability of the modified pyrrole monomers with its large hydrophilic side group to diffuse into the hydrogel. The primary method of controlling the cross-link density within the hydrogel is to either vary the weight percent of the PEGDMA cross-link relative to the methacrylamide monomer or vary the molecular weight of the PEG within the PEGDMA cross-link. As it was noted in Chapter 2, the flexibility of the hydrogel decreased as the molecular weight of the PEG decreased in the PEGDMA cross-link.

The degree of swelling can also affect the mechanical properties of the hydrogel. The force exerted by the swelling of the hydrogel is a result of the hydrophilic affinity of the hydrogel to water and equilibrium established within the networked cross-linked 
structure. Therefore the swelling properties of the hydrogel may be modified by copolymerizing with monomers of varying hydrophobicity. Therefore, the hydrophilic nature of the hydrogel needs characterization. This observation should be performed as a function of temperature because it has been observed that hydrophilicity may increase as temperature increases due to breaks in hydrogen bonds [83]. Hydration or water content measurement for swelling and rehydration may be determined by equilibrium water content (EWC) [84]. For this technique, dried samples may be quantified against hydrated samples at equilibrium. Both the measurements for dehydration and rehydration should be quantified to characterize swelling behavior.

\subsection{Improve the Yield on the Synthesis of Hydrophilic Pyrrole Salt}

The organic synthesis techniques need improvement and changes to improve the yield on the synthesis of the hydrophilic pyrrole salt. The most critical step where yield could be improved is in the sulfonation and deprotection step of the synthesis method. It was noted that in the sulfonation step there may be several reactions occurring simultaneously. Therefore, the provided reaction time for the modified pyrrole may not be adequate enough to complete among the other competing reactions. A future step to improve yield is to separate several of the reactions so that they successively are complete before proceeding to the following reaction.

\subsection{Increase Polypyrrole Concentration in the Hydrogel}

It was demonstrated that the polymerization of pyrrole into the hydrogel may be increased by modifying the hydrophobic pyrrole monomer with a hydrophilic group. A 
simple attempt to increase the diffusion of pyrrole into the hydrogel may be to increase the concentration of the hydrophilic pyrrole salt in the electrochemical cell. Another possibly is to modify the pyrrole monomer with a smaller hydrophilic side group. It is possible that a large side group may prohibit polymerization of PPy. It was shown that the $\% \mathrm{~T}$ of polypyrrole in the hydrogel using the carbonated pyrrole was slightly lower than that of the sulfonated pyrrole. It is plausible that modification of the pyrrole with higher hydrophilic tendencies may promote faster diffusion or higher concentrations of pyrrole into the hydrogel. The potential of other hydrophilic side groups should be investigated.

\subsection{Enhancement of Conductivity by Gold Nanoparticles}

Initial attempts at incorporating gold nanoparticles into the hydrogel to enhance conduction were not successful. Conduction of the hydrogel may have highly been dependent on the nanoparticle size, concentration, and distribution within the hydrogel. Inadequate dispersion due to agglomeration of the nanoparticles and large nanoparticle size may have prevented conduction enhancement. However, it still may be possible to enhance conduction of the polymer if only gold nanoparticle agglomeration before and during polymerization in can be minimized. To promote better dispersion of gold nanoparticles throughout the polymer, it may be possible that the gold nanoparticles are better distributed in the copolymer if it is grown in-situ.

In a modified approach to enhance the conductivity of the polymer, gold nanorods may be more advantageous. If particles are used, the path of conduction may be more tortuous than if rods are used. Rods may provide a more direct path of conduction but it 
may depend on the diameter and the separation distance between the rods. It may be advantageous to use a wire mesh to provide a uniform and fast conduction over the entire polymer compound. Additionally, it may be an advantage to use a mix of both particles and rods to achieve the fast conduction through the gel that will minimize the voltage needed.

\subsection{Effects of the Electrolytic Solution}

The human body is full electrolytes and the types of electrolytes vary throughout different parts and regions. If this polymer compound were to be placed in vivo, the effect on the exposure to those electrolytes found within that region of the body will need to be identified. These factors may have an impact on the response of the device when an electrical current is applied for actuation $[85,86]$.

\subsection{Alternative Methods to Reduce Organics}

Several synthesis and polymerization methods and techniques were specifically chosen to minimize the use of organic compounds. This was consciously done to reduce issues with organic materials within the polymer compound causing a decline in biocompatibility. Alternative and better techniques and/or methods to minimize organic compound throughout the future development of the polymer compound needs to be studied to promote enhanced biocompatibility. 


\subsection{Identify the Influences of Actuation by Electrical Stimulation}

The amount of displacement or contraction needs to be quantified for the polymer compound based on the amount of electric current or field used for actuation. The capacitance of the polymer compound should be measured to determine the amount of electric potential the polymers can hold and what amount of power may be required to actuate the EAP-hydrogel. Electroactive polymers will not be able to change shape or if they cannot hold charge. These measurements may be performed on the VSP with cyclic voltammetry experiments. The measurements will occur between a set negative and positive potential at various scan rates $(\mathrm{mV} / \mathrm{s})$.

The temperature and possible thermal conduction of the polymer compound will also be examined. It is possible that heating through electrical conduction may aid or hinder the actuation of the polymer composite. If thermal conduction may aid in actuation of the composite, it may be useful to utilize the gold nanoparticles to control or direct conduction and heating throughout the polymer composite.

\subsection{Biocompatibility Studies}

The use of organic chemicals and compounds in the synthesis and development of this polymer compound is not completely avoidable. Therefore, there may be trace elements of organics that may leach out from the material. It is important that the polymer compound be analyzed for biocompatibility to identify the possible hazards. For the future the protocol needs further optimization to promote reproducible and significant measurements of toxicity of polymers to the cells so we obtain accurate and precise 
indication of biocompatibility. The cell concentration density is important to study because it may be possible that a very high concentration of cells may proliferate so well that it may mask any toxicity.

The protocol for the MTT assay will need to be refined for reproducible and significant measurements of toxicity to the cells. The optimal cell concentration density in the sample wells needs to be studied further so that samples treated with the cells show the smallest levels of toxicity if present. It is possible that a well with a very high concentration of cells may proliferate so well that it may mask any toxicity. The length of time the materials will be exposed and treated on the cell needs to be studied to allow sufficient time for possible toxins to leach from the polymer samples if it is present. The length of time for cell growth following treatment needs to be studied to allow sufficient time for the death of cells that may be cytotoxically affected. These studies will promote a more accurate and precise indication of biocompatibility of the polymer samples synthesized. 


\section{REFERENCES}

1. Heath Care Diary. "Congestive Heart Failure". Web Image. Congestive Heart Failure. 2005. 5 March 2010. <http://healthcarediary.com/wpcontent/uploads/2011/07/Congestive-Heart-Failure-150x150.jpg>.

2. McClintic JR, Physiology of the Human Body, 2nd Ed., John Wiley \& Sons, Inc., 1978, p 314-343.

3. Kumar V, Abbas AK, Fausto N, Robins \& Cotran Pathologic Basis of Disease 7th ed., Elsevier Inc., 2005, Philadelphia, p 560-615.

4. U.S. Department of Health and Human Services, NHLBI, Congestive Heart Failure Data Fact Sheet: NHLBI, Bethesda, MD, NHLBI, September 1996, Online, Internet, February 1, 2008, Available: http://wrongdiagnosis.com/artic/nhlbi_congestive_heart_failure_data_fact_sheet_ nhlbi.htm.

5. Neubauer $\mathrm{S}$, The failing heart - an engine out of fuel, $\mathrm{N}$ Engl J Med, 2007, 356 (11): p 1140-51.

6. CDC, National Center for Chronic Disease Prevention \& Health Promotion, Facts About Heart Failure in Older Adults: CDC-OC, Atlanta, GA, March 5, 1999, Online, Internet, February 1, 2008, Available: http://wrongdiagnosis.com/artic/facts_about_heart_failure_in_older_adults_cdc_o c.htm. 
7. Salmons S, Jarvis JC, Cardiac assistance from skeletal muscle: a critical appraisal of the various approaches, British Heart Journal, 1992, 68, p 333-8.

8. Chachques JC, Grandjean PA, Carpentier A. Latissimus dorsi dynamic cardiomyoplasty, Ann Thorac Surg, 1989, 48, p 600-4.

9. Grandjean P, Austin L, Chan S, et al. Dynamic cardiomyoplasty: Clinical follow-up results. J Card Surg 1991, 6, p 80-8.

10. Moreira LFP, Stolf NAG, Jatene AD. Hemodynamic benefits of cardiomyoplasty in clinical and experimental myocardial dysfunction. In: RC-J Chiu, I Bourgeois, eds. Transformed Muscle for Cardiac Assist and Repair Mount Kisco, NY: Futura. 1990, p179-88.

11. Lee KF, Dignan RJ, Parmar JM, et al. Effects of dynamic cardiomyoplasty on left ventricular performance and myocardial mechanics in dilated cardiomyopathy, JThorac Cardiovasc Surg 1991, 102, p 124-31.

12. Capouya ER, Gerber RS, Drinkwater DC, et al. Girdling effect of nonstimulated cardiomyoplasty on left ventricular function. Ann Thorac Surg 1993, 56, p 867-71.

13. Mannion JD, Magno MG, Buckman PD, et al. Acute electrical stimulation increases extramyocardial collateral blood flow after a cardiomyoplasty. Ann Thorac Surg 1993, 56, p 1351-8.

14. Chachques JC and Carpentier A, 1st Virtual Congress of Cardiology. "Cardiomyoplasty technique". Web Image. Cardiomyoplasty for chronic heart failure. 10 Feb 2010. 30 Nov 2011. $<$ http://www.fac.org.ar/cvirtual/cvirteng/cienteng/cceng/ccc1519i/ichachq/fig $1 . j p$ g>. 
15. McCarthy PM, New surgical options for the failing heart, J Heart Valve Dis. 1999, $8(5), \mathrm{p} 472-475$.

16. Batista RJV, Santos JLV, Takeshita N, et al. Partial left ventriculectomy to improve left ventricular function in end-stage heart disease. J Card Surg. 1996, 11, p 96-97.

17. Batista R. Partial left ventriculectomy--the Batista procedure. Eur J Cardiothorac Surg., 1999, Suppl 1:S12-S19; S39-S43.

18. Surgical Associates of Texas, Left Ventricular Reduction, Houston, Texas, 2000, Online, Internet, March 5, 2010, Available: http://texheartsurgeons.com/LVRsurgery.htm.

19. Surgical Associates of Texas, "Batista". Web Image. Left Ventricular Reduction, 2000. 5 Mar 2010. < http://texheartsurgeons.com/Images/batistal.jpg>.

20. Heart Failure Online, "Left Ventricular Assist Device". Web Image, Left Ventricular Assist Device. 2010. 5 Mar 2010. < http://www.heartfailure.org/images/img_lvad_diag.jpg>.

21. Starling RC, McCarthy PM, Buda T, et al. Results of partial left ventriculectomy for dilated cardiomyopathy: Hemodynamic, clinical and echocardiographic observations. J Am Coll Cardiol. 2000, 36(7), p2098-2103.

22. American Heart Association, Left Ventricular Assist Device, Online, Internet, February 4, 2008, Available: http://www.americanheart.org/presenter.jhtml?identifier $=4599$. 23. American Heart Association, Heart Transplants: Statistics, Online, Internet, February 4, 2008, Available: http://www.americanheart.org/presenter.jhtml?identifier=4588. 
24. Pepas NA, Bures P, Leobandung W, Ichikawa H, Hydrogels in pharmaceutical formulations, European Journal of Pharm Biopharm, 2000, 50, p 27-46.

25. MacCallum JR and Vincent CA, Polymer Electrolyte Reviews, Elsevier Applied Science, London, Vol 1 and 2, 1987 and 1989.

26. Callister WD. , Materials Science and Engineering an Introduction, 5th Ed., John Wiley \& Sons, Inc., New York, 2000, p 521-638.

27. Skotheim, T., Elsenbaumer, R., Reynolds, J., Eds.; Handbook of Conducting Polymers, 2nd ed.; Marcel Dekker, Inc.: New York, NY, USA, 1998.

28. Herbert Naarmann "Polymers, Electrically Conducting" in Ullmann's Encyclopedia of Industrial Chemistry 2002 Wiley-VCH, Weinheim.

29. Unur E, Beaujuge PM, Ellinger S, Jung JH, and Reynolds JR, Black to Transmissive Switching in a Pseudo Three-Electrode Electrochromic Device, Chem. Mater. $2009,21,5145-5153$.

30. Yoseph Bar-Cohen, Electrochemistry Encyclopedia. "A gripper", Web Image, Electroactive Polymer (EAP). Dec 2004, 20 Oct 2010. $<$ http://electrochem.cwru.edu/encycl/fig/p02/p02-f03.png $>$.

31. Y. Bar-Cohen, Electroactive Polymers as Artificial Muscles - Reality and Challenges, Proceedings of the 42nd AIAA Structures, Structures Dynamics and Materials Conferences (SDM), Gossamer Spacecraft Forum (GSF), held in Seattle WA, April 16-19, 2001.

32. IUPAC. "Glossary of basic terms in polymer science (IUPAC Recommendations 1996)", PureAppl. Chem., 1996, 68, p 2287-2311. 
33. Sperling LH, Interpenetrating Polymer Networks: An Overview; In Interpenetrating Polymer Networks Klempner, D., et al., Advances in Chemistry; American Chemical Society: Washington, DC, 1994, p 3-38.

34. INTERNATIONAL ORGANIZATION FOR STANDARDIZATION, in "Biological Evaluation of Medical Devices - Part 5: Tests for In Vitro Cytotoxicity (ISO 10993-5:2009).

35. INTERNATIONAL ORGANIZATION FOR STANDARDIZATION, in "Biological Evaluation of Medical Devices - Part 12: Sample Preparation and Reference Materials (ISO 10993-5:2007).

36. Van der Meulen I, Geus M, Antheunis H, Deumens R, Joosten EAJ, Koning CE, and Heise A, Polymers from Functional Macrolactones as Potential Biomaterials: Enzymatic Ring Opening Polymerization, Biodegradation, and Biocompatibility, Biomacromolecules, 2008, 9; p 3404-3410.

37. Pepas NA, Bures $\mathrm{P}$, Leobandung W, Ichikawa H, Hydrogels in pharmaceutical formulations, European Journal of Pharm Biopharm, 2000, 50, p 27-46.

38. Lee JH and Oh SH, MMA/MPEOMA/VSA copolymer as a novel blood-compatible material: Effect of PEO and negatively charged side chains on protein adsorption and platelet adhesion, Journal of Biomedical Materials Research, 2002, 60, p 4452.

39. Lowery TH and Richardson KS, Mechanism and Theory in Organic Chemistry, Harper Collins Publishers 3rd ed. 1987, p 177.

40. Litwinienko G, Beckwith ALJ and Ingold KU, The frequently overlooked importance of solvent in free radical syntheses Chem. Soc. Rev., 2011, 40, p 2157-2163. 
41. Avila DV, Brown CE, Ingold KU and Lusztyk J, Solvent effects on the competitive beta.-scission and hydrogen atom abstraction reactions of the cumyloxyl radical. Resolution of a long-standing problem, ,J. Am.Chem. Soc., 1993, 115, p 466-470.

42. Catallo WJ, Feix JB, Gale RJ, Movement of methylphenazyl free radicals in polar and nonpolar liquids, Free Radic Biol Med. 1992, 13(1), p 35-40.

43. Satoh K, Sakagami H, Terasaka H, Ida Y, Fujisawa S., Interaction between hydroquinone and ascorbic acid derivatives: quenching effect of organic solvents., Anticancer Res. 2000 May-Jun, 20(3A), p 1577-81.

44. Ohta Y, Inoue Y, Chujo R, Kishimoto M, and Seo I, Microstructure of vinylidene cyanide copolymers with linear-chain fatty acid vinyl esters, Polymer, 1990, 8, p $1581-1588$.

45. Lin-Gibson S, Bencherif S, Cooper JA, Wetzel SJ, Antonucci JM, Vogel BM, Horkay F and Washburn N, Synthesis and Characterization of PEG Dimethacrylates and Their Hydrogels, Biomacromolecules, 2004, 5, p1280-1287.

46. S. Jana, A. Salehi-Khojin, W. H. Zhong, H. Chen, X. Liu, Q. Huo, Effects of gold nanoparticles and lithium hexafluorophosphate on the electrical conductivity of PMMA, Solid State Ionics, 2007; 178:1180-1186.

47. Link S and El-Sayed MA, Shape and Size dependence of radiative, non-radiative and photothermal properties of gold nanocrystals, International Reviews in Physical Chemistry, 2000, 19(3), p 409-453. 
48. R. A. Frimpong, S. Fraser, J. Z. Hilt, Synthesis and temperature response analysis of magnetic-hydrogel nanocomposites, J Biomed Mater Res: Part A, 2006, 80A, p $1-6$.

49. Zhang, QM, Bharti V, Kavarnos G, Schwartz M, "Poly (Vinylidene Fluoride) (PVDF) and its Copolymers", Encyclopedia of Smart Materials, Volumes 1-2, John Wiley \& Sons, 2002, p 807-825.

50. Vinogradov A, Schwartz M (Ed.), (2002). "Piezoelectricity in Polymers", Encyclopedia of Smart Materials, Volumes 1-2, John Wiley \& Sons, 2002, p 780792.

51. Bar-Cohen Y and Leary S, Electroactive Polymers (EAP) Characterization Methods, Proceedings of SPIE's 7th Annual International Symposium on Smart Structures and Materials, SPIE, Newport, CA, March 1-5, 2000; Paper No. 3987-04.

52. Salaneck WR, Clark DT, Samuelsen EJ, Science and Applications of Conduction Polymers, Adam Hilger, Bristol, 1999.

53. Bolto BA, McNeill R and Weiss DE, Electronic Conduction in Polymers. III. Electronic Properties of Polypyrrole, Australian Journal of Chemistry, 1963, 16(6), p $1090-1103$.

54. Ramanaviciene A and Ramanavicius A, Application of polypyrrole for the creation of immunosensors, Crit. Rev. Anal. Chem., 2002, 32(3), p 245-252.

55. Hammerle M, Schuhmann W, Schmidt HL, Amperometric polypyrrole enzyme electrodes: effect of permeability and enzyme location, Sens. Actuators B, 1992; $6, \mathrm{p} 106$. 
56. Kassim A, Ekarmul Mahmud HNM, L. M. Yee LM, Hanipah N, Electrochemical Preparation and Characterization of Polypyrrole-Polyethylene Glycol Conducting Polymer Composite Films, The Pacific Journal of Science and Technology, 2006, 7(2), p 103-107.

57. Zhang A, Liu Y, Wang H, Zhang G, Zhang K, Lu J, Electrochemical Synthesis of Polypyrrole in a Room Temperature Ionic Liquid and its Properites, Chinese Journal of Chemistry, 2009, 27(2), p248-252.

58. Zhou Y, Yu M, Li S, Li L, Electropolymerization of polypyrrole films doped with sulfonated polyaniline, The European Physical Journal Applied Physics, 2008, 42, p 141-144.

59. Hutchison AS, T. W. Lewis TW, Moulton SE, Spinks GM, and Wallace GG, Development of polypyrrole-based electrochemical actuators, Synthetic Metals, 2000, 113, p121-127.

60. Spinks GM, Wallace GG, Liu L and Zhou D, Conducting Polymers and Carbon Nanotubes as Electrochemical Actuators and Strain Sensors, Mat. Res. Soc. Symp. Proc., 2002, 698, p 5-16.

61. Wu Y, Alici G, Spinks GM and Wallace GG, Fast Trilayer polypyrrole bending actuators for high speed applications, Synthetic Metals, 2006; 156, p 1017-1022.

62. Ding J, Liu L, Spinks GM, Zhou D, Wallace GG and Gillespie J, High performance conducting polymer actuators utilizing a tubular geometry and helical wire interconncects, Synthetic Metals, 2003, 138, p 391-398.

63. Tso CH, Madden JD and Michal CA, An NMR study of PF6- ions in polypyrrole, Synthetic Metals, 2007, 157, p 460-466. 
64. Madden JD, Cush RA, Kanigan TS, Brenan CJ and Hunter IW, Encapsulated polypyrrole actuators, Synthetic Metals, 1999, 105, p 61-64.

65. Yamaura M, Satao K, Hagiwara T, Iwata K, Memory effect of electrical conductivity upon the counter-anion exchange of polypyrrole films, Synthetic Metals, 1992, 48, p 337-354.

66. Izadi-Najafabadi A, Tan TH and Madden JD, Towards High Power Polypyrrole/Carbon Capacitors, Synthetic Metals, 2005, 152, p 129-132.

67. Madden JD, Rinderknecht D, Anquetil PA and Hunter IW, Creep and cycle life in polypyrrole actuators, Sensors and Actuators A, 2007, 133, p 210-217.

68. Madden JD, Cush RA, Kanigan T.S and Hunter IW, Fast contracting polypyrrole actuators, Synthetic Metals, 2000, 113, p 185-192.

69. Yamaura M, Hagiwara T, Iwata K, Enhancement of Electrical Conductivity of Polypyrrole Film by Stretching: Counter Ion Effect., Synthetic Metals, 1998, 26, p 209-224.

70. Yoon CO, Sung HK, Kim JH, Barsoukov E, Kim JH, and Lee H, The effect of lowtemperature conditions on the electrochemical polymerization of polypyrrole films with high density, high electrical conductivity and high stability, Synthetic Metals, 1999; 99, p 201-212.

71. Hu CC and Lin XX, Ideally Capacitive Behavior and X-Ray Photoelectron Spectroscopy Characterization of Pyrrole: Effects of Polymerization Temperatures and Thickness/Coverage, Journal of The Electrochemical Society, $2002 ; 149(8): 1049-1057$. 
72. Standard Practice for General Techniques for Obtaining Infrared Spectra for Qualitative Analysis, ASTM International, 2002, E, p 1252-98.

73. Saville P, Polypyrrole Formation and Use, Defense Research and Development Canada, Jan 2005.

74. Chen W, Xingwei L, Gi X, Zhaoquang W and Wenquing Z, Appl. Surf. Sci., 2003, $218, \mathrm{p} 216$.

75. Standard Practice for General Techniques for Obtaining Infrared Spectra for Qualitative Analysis, ASTM International, , 2002, E, p 1252-98.

76. P. Saville, Polypyrrole Formation and Use, Defense Research and Development Canada, Jan 2005.

77. Wang Y, Qian W, Tang Y, Ding S and Zhang H, Direct electrochemistry and electroanalysis of hemoglobin adsorbed in self-assembled films of gold nanoshells, Talanta, 2007, 72, p 1134-1140.

78. Li MD, Cul Y, Gao MX, Luo J, Ren B and Tian ZQ, Clean Substrates Preparted by Chemical Adosorption of Iodide Followed by Electrochemical Oxidation for Surface-Enhanced Raman Spectroscopic Study of Cell Membrane, Analytical Chemistry, 2008, 80 (13), p 5119-5125.

79. Green TW., Protective Groups in Organic Synthesis, John Wiley \& Sons, New York, 1981, p 1 .

80. Kocienski PJ, Protecting Groups, New York, Georg Thieme Verlag, 2000, 2, p 20912 
81. Anderson HJ, Loader CE, Xu RX, Le N, McDonald NJ, Edwards LG, Pyrrole chemistry. XXVIII. Substitution reactions of 1-(phenylsulfonyl)pyrrole and some derivatives, Canadian Journal of Chemistry, 1985, 63, p 896-902.

82. Havinga EE, ten Hoeve W, Meijer EW and Wynbert H, Water-Soluble Self-Doped 3Substitute Polypyrroles, Chemistry of Materials, 1989, 1, p 650-659.

83. Satarkar NS and Hilt JZ, Hydrogel nanocomposites as remote-controlled biomatierals, Acta Biomaterialia, 2008, 4, p 11-16.

84. Kim BC, Spinks G, Too CO, Wallace GG, Bae YH, Preparation and characterization of processable conducting polymer-hydrogel composites, Reactive \& Functional Polymers, 2000, 44, p 31-40.

85. Kim HI, Park SJ, Kim SI, Kim NG, and Kim SJ, Electroactive polymer hydrogels composed of polyacrylic acid and poly (vinyl sulfonic acid) copolymer for application of biomaterial, Synthetic Metals, 2005, 155, p 674-676.

86. Yuk SH and Lee HB, Electric-current-sensitive polymers. Reversible bending of rodshaped acrylamide gel in naci solution, Journal of Polymer Science Part B:

Polymer Physics, 1993; 31(4), p487-489. 


\section{APPENDICES}

Appendix A. Illustration of Photoinitiator Free Radical Generation.

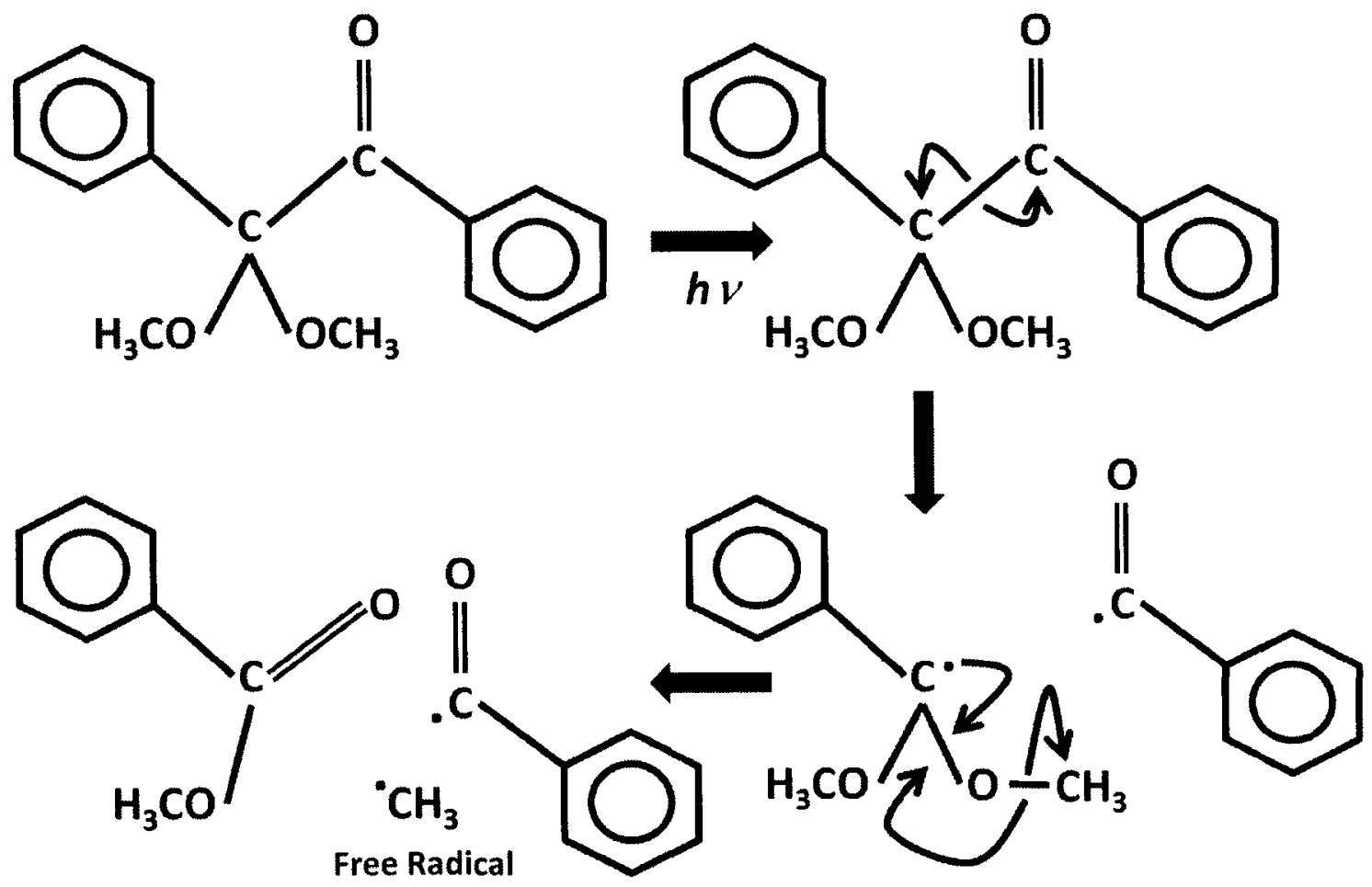


Appendix B. Protocol for the Bioactivity Assay.

Appendix B1. L929 Cell Growth and Preparation on 96-Well Plates

The L929 cell line was cultured in DMEM with $10 \%$ supplemental calf serum, $1 \%$ glutamine, and $1 \%$ antibiotics (penicillin and streptomycin). The cells were cultured in an incubator kept at $5 \% \mathrm{CO}_{2}$ atmosphere at $37^{\circ} \mathrm{C}$. After growth, cells were detached from the culture dish with trypsin. Trypsin is a protease that hydrolyses the membrane proteins that aid in cell adhesion to the plate. The detached cells were counted using a hemocytometer. Once the cell concentration was determined, cell concentrations of $1,000,5,000$, and 10,000 were dispensed in a third of 96 -well plate to determine the proper cell concentration for $100 \%$ confluent growth for the MTT assay. Our preliminary results showed that a cell concentration of 10,000 cells/well provided $100 \%$ confluence with cell growth overnight. This was an ideal cell concentration for the assay which provided the $100 \%$ confluence cell growth necessary for the biocompatibility assay with MTT. Error! Reference source not found. shows an illustration of $100 \%$ confluent growth with cell-to-cell contact for proper sample treatment. This is the appearance that was indicative of our cells before treatment and as a non-cytotoxic (negative) response in the elution test method. . The cells were allowed to grow 24 hours in the wells prior to any treatment. 


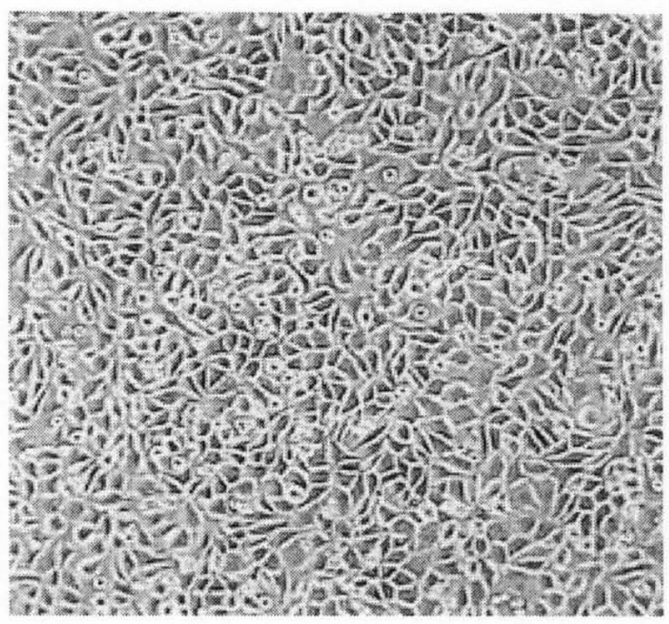

Figure 57. Image of $100 \%$ confluence for cell growth.

Appendix B2. Protocol for the MTT Assay

Two 96-well plates were made to determine if continued cell growth following treatment was necessary to allow cells affected by toxicity to lose viability before the MTT was added. One plate would have MTT added immediately after treatment (24-0) and the second plate would have MTT added 24 hours following treatment (24-24). The cells would be allowed to grow in growth media overnight at $5 \% \mathrm{CO}_{2}$ atmosphere at $37^{\circ} \mathrm{C}$.

The polymer samples were cut into $3 \mathrm{~mm} \times 3 \mathrm{~mm}$ squares and placed in deionized (DI) water. The polymers were then steam autoclaved at $121^{\circ} \mathrm{C}$ for 20 minutes to sterilize the samples so that they would not contaminate the cells with mold or bacterial.

Following treatment, the samples and media were dumped. MTT was then pipetted into the $24-0$ plate the wells and incubated at $37^{\circ} \mathrm{C}$ for 2 hours. The $24-24$ plate would be given growth media and incubated for an additional 24 hours in at $5 \% \mathrm{CO}_{2}$ atmosphere at $37^{\circ} \mathrm{C}$ before MTT was added. After incubation, DMSO was added into 
each well and incubated at $37^{\circ} \mathrm{C}$ for 1 hour to allow the formazan crystals to dissolve. The plate was then taken out of the incubator, covered, and placed on a rocker for 1 hour to enable the purple coloration to mix well before absorbances were read at $560 \mathrm{~nm}$. 


\begin{tabular}{|c|c|c|c|c|c|c|}
\hline Group & Electrolyte & Blectrode (WE/CE/RE) & $\begin{array}{l}\text { Current Density } \\
\left(\mathrm{mA} / \mathrm{cm}^{2}\right)\end{array}$ & $\begin{array}{l}\text { Temp } \\
(\mathrm{O} C)\end{array}$ & Time (hr) & \multirow{12}{*}{ 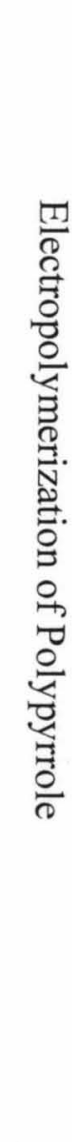 } \\
\hline A Kassim (2006) & $0.05-0.3 \mathrm{M} \mathrm{p}-\mathrm{TS}$ & ITO/Carbon Rod/SCE & $1.2 \mathrm{~V}$ & $? ?$ & $2 \mathrm{hr}$ & \\
\hline$J \operatorname{Lu}(2009)$ & bmimPF $_{6}$ & ITO/Pt wire/Ag-AgCl & 0.2 & RT & ?? & \\
\hline GM Spinks (2000) & $0.1 \mathrm{M}$ pTS & $\mathrm{SS} / ? ? / \mathrm{Ag}-\mathrm{AgCl}$ & 0.1 & $? ?$ & $30-60 \min$ & \\
\hline GM Spinks (2002) & $0.05 \mathrm{M}$ TBAP in PC & Pt & 0.15 & -28 & ?? & \\
\hline GM Spinks (2003) & 0.05M TBAP in PC & Pt wire/SS mesh/?? & 0.15 & -28 & 6 & \\
\hline GM Spinks (2006) & $0.05 \mathrm{M}$ TBAP in PC \& $0.5 \%$ distilled $\mathrm{H} 20$ & gold coated PVDF membrane & 0.1 & $-25,-28$ & 12 & \\
\hline JD Madden (1999) & 0.05M TEAP in PC \& $1 \%$ distilled $\mathrm{H} 20$ & $\mathrm{GC} / \mathrm{Pt}$ foil/Ag-AgCl & 0.125 & -30 & 24 & \\
\hline JD Madden (2000) & 0.05M TEAP in PC \& $1 \%$ distilled $\mathrm{H} 20$ & $\mathrm{GC} / \mathrm{Pt}$ foil/ $\mathrm{Ag}-\mathrm{AgCl}$ & 0.125 & -30 & 24 & \\
\hline JD Madden (2005) & $0.05 \mathrm{M}$ TEAP in PC \& $1 \%$ distilled H2O & CFP/CFP/?? & 0.1 & -30 & 24 & \\
\hline JD Madden (2007) & 0.06M TEAP in PC \& $1 \%$ distilled $\mathrm{H} 20$ & $\mathrm{GC} / \mathrm{Pt}$ foil/Ag-AgCl & 0.125 & -40 & 24 & \\
\hline JD Madden (2007) & 0.05M TEAP in PC \& $1 \%$ distilled $\mathrm{H} 20$ & Glassy carbon/copper & 0.125 & -30 & 8 & \\
\hline
\end{tabular}




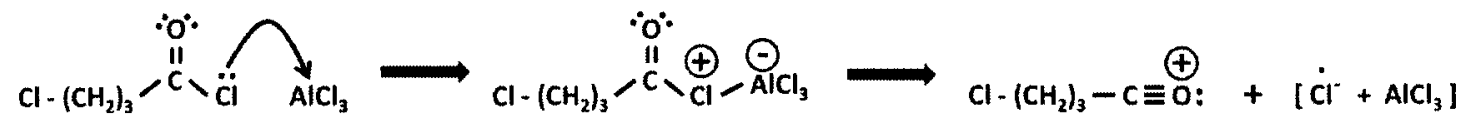
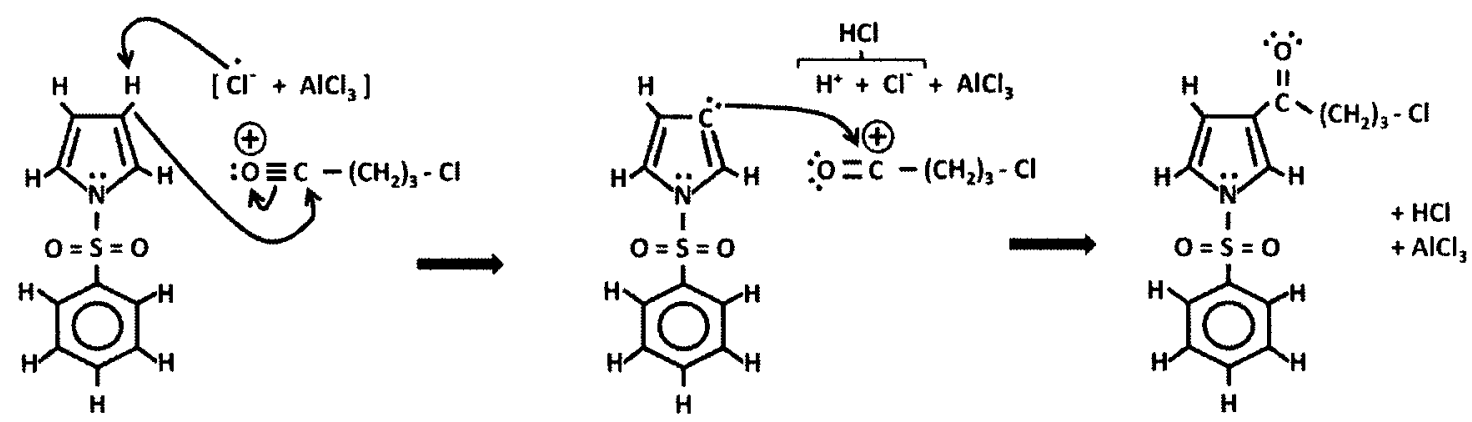
Appendix E. Clemmensen Reduction Mechanism
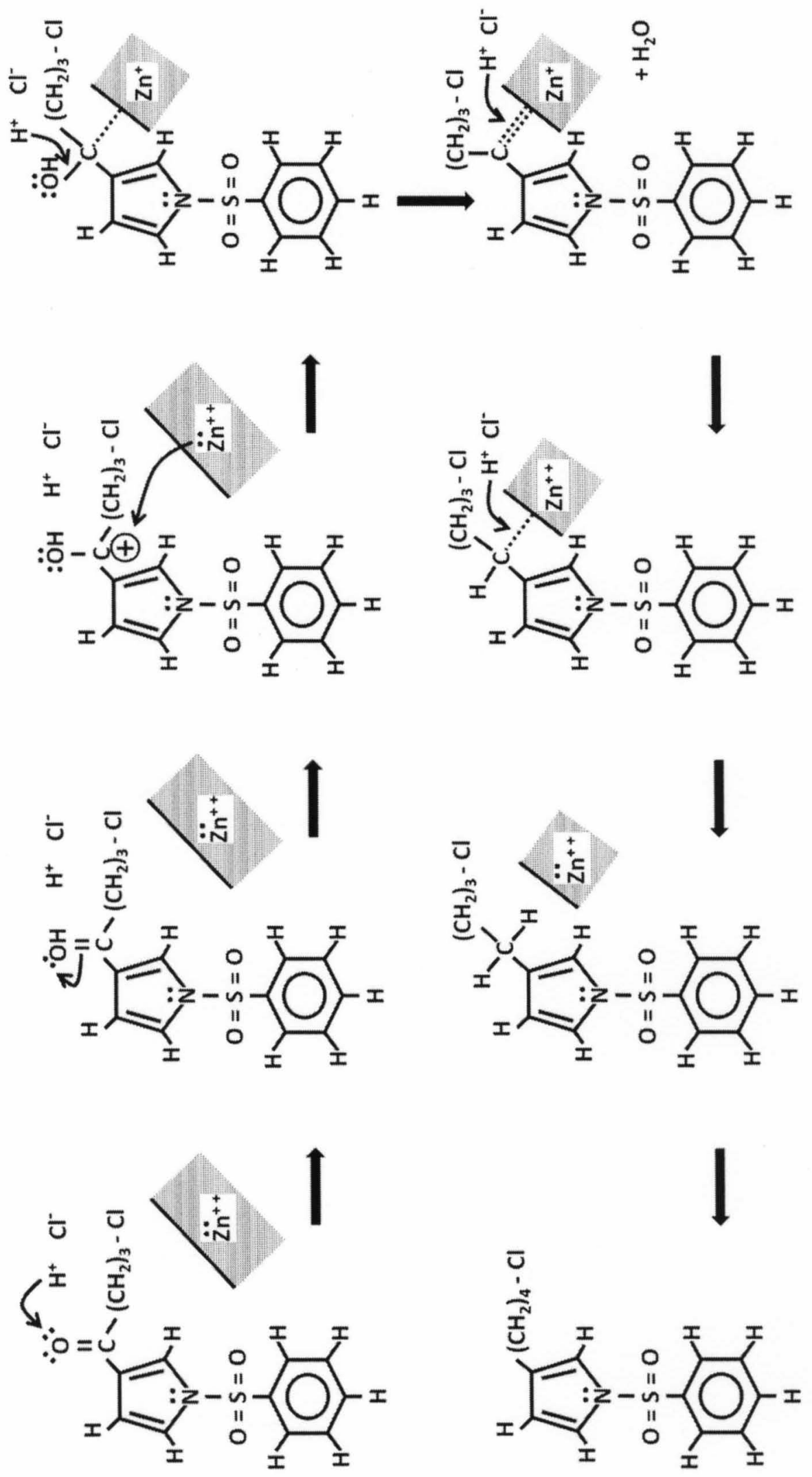


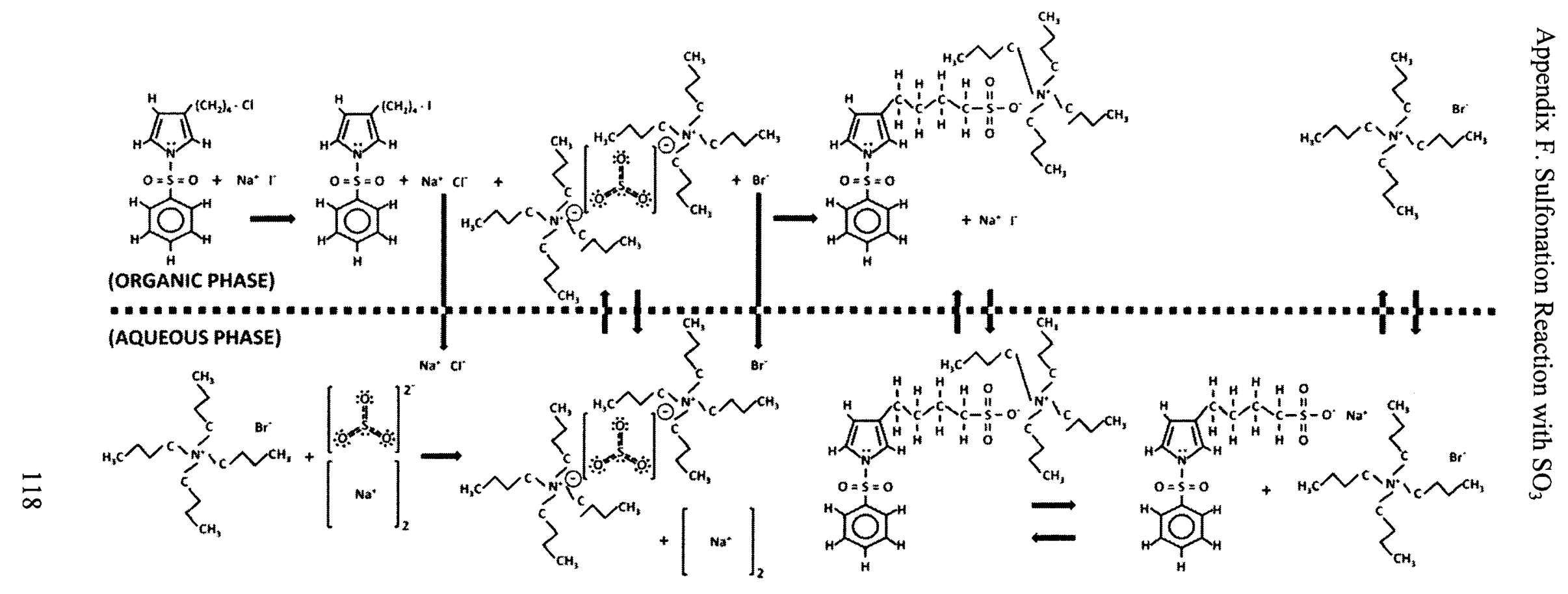




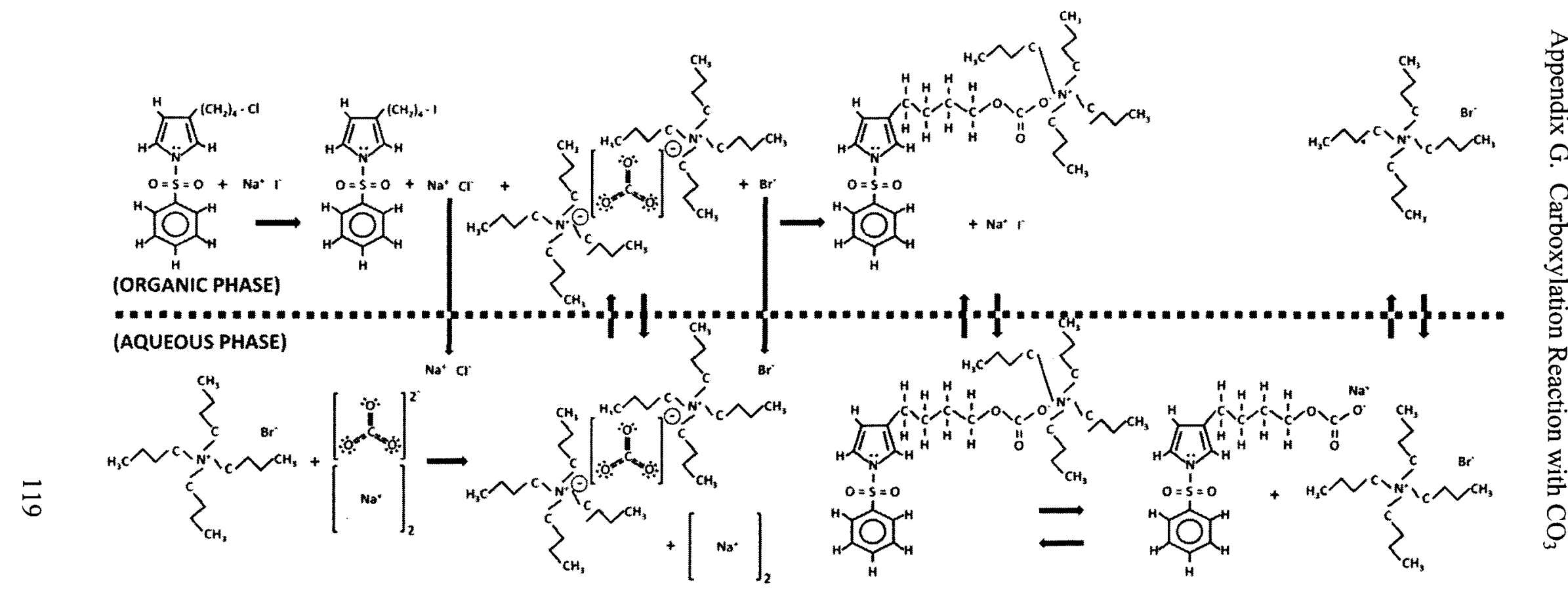


Appendix H. Deprotection Mechanism
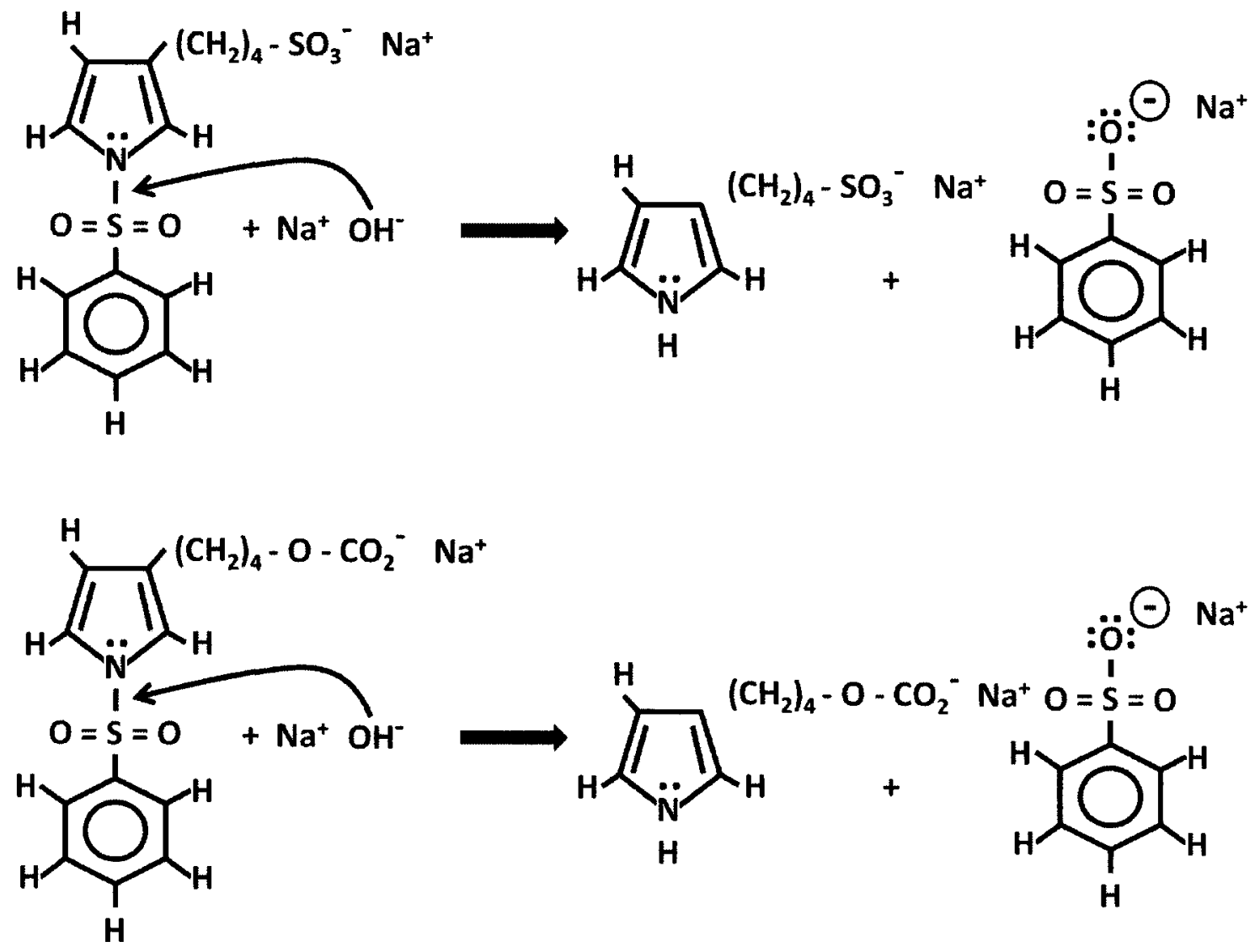


\section{CURRICULUM VITAE}

NAME: James J. Lee

ADDRESS: Department of Chemical Engineering

Room 206 A, Ernst Hall

University of Louisville

Louisville, KY 40208

DOB: $\quad$ Baltimore, MD - June, 15, 1978

EDUCATION

\& TRAINING: B.S., Chemical Engineering

Virginia Polytechnic Institute and State University

1996-2001

Study Abroad; Chemical Engineering Unit Operations Lab

University College London

London, England

Summer 2000

Ph.D. Graduate Student, Chemical and Biochemical Engineering

University of Maryland Baltimore County

2002-2004

Ph.D., Chemical Engineering

University of Louisville

2004-2011

AWARDS \& HONORS:

Kentucky Space Grant Consortium Graduate Fellowship

August 2009-December 2010

Engineering Expo, University of Louisville, Louisville, KY 
Graduate Overall $2^{\text {nd }}$ Place, Poster, 2010.

Best in Chemical Engineering Dept., Poster, 2010.

Overall Winner M.S./Ph.D. Poster Competition, 2007

$1^{\text {st }}$ Place, Chemical Engineering Dept., Poster Competition, 2007

$2^{\text {nd }}$ Place, Chemical Engineering Dept. Poster Competition, 2005

The Britton Chance Award, $33^{\text {rd }}$ International Society on Oxygen Transport to Tissue, "Presented for the scientific excellence of the individual's first authored manuscript on the topic of oxygen transport under the age of 30 years," 2005

The Dietrich Lübbers Award, $31^{\text {st }}$ International Society on Oxygen Transport to Tissue, "Presented for the most outstanding manuscript submitted by an investigator under the age of 30 years," August 20, 2003.

Golden Key International Honour Society, University of Louisville Chapter The Princeton Review Literacy Award for 2008, "Recognition of involvement in literacy-based initiatives," 2008

Graduate Scholarship Award, 2007

Institute for Molecular Diversity and Drug Design $\left(\mathrm{IMD}^{3}\right)$, University of Louisville Award, 2005 $3^{\text {rd }}$ Place in Poster Competition, 2007

Research! Louisville, Louisville, KY

Honorable Mention for Doctoral Basic-Science, Student Division, 2006.

Virginia Tech's Academic Dean's List, Fall 2000, Spring 2001.

\section{PROFESSIONAL AFFILIATIONS:}

American Institute of Chemical Engineers (AIChE)

American Chemical Society (ACS)

Polymeric Materials Science \& Engineering (PMSE)

Biochemical Technology (BIOT),

Biomedical Engineering Society (BMES)

Sigma Xi $(\Sigma \Xi)$, Scientific Research Society 
International Society of Oxygen Transport to Tissue (ISOTT)

Golden Key International Honour Society

Alpha Chi Sigma, Professional Chemistry Fraternity ( $\Gamma I$ Chapter)

\section{PUBLICATIONS:}

James J. Lee, Duane F. Bruley and Kyung A. Kang, "Manipulation of the affinity between protein and metal ions by imidazole and $\mathrm{pH}$ for metal affinity purification of Protein C form Cohn Fraction IV-1," Advances in Experimental Medicine and Biology: Oxygen Transport to Tissue XXIX, 578, Springer-Verlag, NY, 2008.

James J. Lee, Duane F. Bruley and Kyung A. Kang, "Effect of pH and Imidazole on Protein C Purification from Cohn Fraction IV-1 by Immobilized Metal Affinity Chromatography," Advances in Experimental Medicine and Biology: Oxygen Transport to Tissue XXVIII, 578, Springer-Verlag, NY, 2007.

James J. Lee and Duane F. Bruley, "Thermodynamic Approach to Optimize Immobilized Metal Affinity Chromatography Purification of Protein C," Advances in Experimental Medicine and Biology: Oxygen Transport to Tissue XXVII, 578, Springer-Verlag, NY, 2006.

James J. Lee, Eileen E. Thiessen and Duane F. Bruley, "Protein C Production; Metal Ion/Protein Interfacial Interaction in Immobilized Metal Affinity Chromatography," Advances in Experimental Medicine and Biology: Oxygen Transport to Tissue XXVI, 566, Springer-Verlag, NY, 2005.

\section{SELECTED PRESENTATIONS:}

James J. Lee and Gerold A. Willing, Biocompatible and Electroactive Polymers, AIChE 2009 Annual Conference, Nashville, TN, November 13, 2009.

James J. Lee and Gerold A. Willing, Characterization of Biocompatible Polymer Composites, AIChE 2008 Annual Conference, Philadelphia, PA, November 19, 2008.

James J. Lee and Gerold A. Willing, Electroactive and Biocompatible Polymers, Southeastern Regional Meeting of the American Chemical Society, Nashville, TN, November 12, 2008. 
James J. Lee, Duane F. Bruley, and Kyung A. Kang, Manipulation of the Adsorption of Factor II, a Protein C Homologue, Using Imidazole During Immobilized Metal Affinity Chromatography Purification of Protein $\mathrm{C}$ from Cohn Fraction IV-1, c San Francisco, CA, November 15, 2006.

James J. Lee, Duane F. Bruley, and Kyung A. Kang, Affinity Modulation Using Imidazole During IMAC Purification of Protein C from Cohn Fraction IV-1, Biomedical Engineering Society 2006 Annual Conference, Chicago, IL, October 12, 2006 (Poster).

James J. Lee, Duane F. Bruley, and Kyung A. Kang, Regulation of Factor II Affinity For Metal Affinity Purification of Protein C from Cohn Fraction IV-1 Using Imidazole and $\mathrm{pH}, 34^{\text {th }}$ Annual International Society on Oxygen Transport to Tissue Meeting, Louisville, KY, August 16, 2006.

James J. Lee, Duane F. Bruley, and Kyung A. Kang, Utilization of Gibbs Free Energy to Optimize the $\mathrm{pH}$ and Imidazole Operating Conditions of Immobilized Metal Affinity Chromatography for Protein C Purification, AIChE 2005 Annual Conference, Cincinnati, OH, November 1, 2005.

James J. Lee, Duane F. Bruley, and Kyung A. Kang, Optimizing an IMA Chromatography Process for Protein C Purification Utilizing Gibb's Free Energy, Biomedical Engineering Society 2005 Annual Conference, Baltimore, MD, September 28 - October 1, 2005 (Poster).

James J. Lee, Duane F. Bruley, and Kyung A. Kang, Effect of $\mathrm{pH}$ and Imidazole on Protein C Purification from Cohn Fraction IV-1 by IMAC, $33^{\text {rd }}$ Annual International Society on Oxygen Transport to Tissue Meeting, Brisbane, Australia, August 28, 2005.

James J. Lee and Duane F. Bruley, Thermodynamics of Interfacial Adsorption in Immobilized Metal Affinity Chromatography of Protein C, Biomedical Engineering Society Annual Conference, Philadelphia, PA, October 14, 2004 (Poster).

James J. Lee and Duane F. Bruley, Thermodynamic Measurement of Interfacial Adsorption in Immobilized Metal Affinity Chromatography of Protein C, $32^{\text {nd }}$ Annual International Society on Oxygen Transport to Tissue Meeting, Bari, Italy, August 23, 2004.

James J. Lee and Duane F. Bruley, Protein C and Copper (II) Metal Ion Interfacial Interaction in Immobilized Metal Affinity Chromatography, American 
Chemical Society Annual Conference, Anaheim, CA, Oral Presentation, April $1,2004$.

James J. Lee, Eileen E. Thiessen, and Duane F. Bruley, Protein C Production; Metal Ion/Protein Interfacial Interaction in Immobilized Metal Affinity Chromatography, $31^{\text {st }}$ Annual International Society on Oxygen Transport to Tissue Meeting, Rochester, NY, August 2003.

James J. Lee and Duane F. Bruley, Protein C Production, Anticoagulant/Antiinflammatory/Antithrombotic; Metal Ion/Protein Interfacial Interaction in Immobilized Metal Affinity Chromatography, Biomed Research Foundation; Second International Interdisciplinary Conference on Cardiovascular Medicine and Science, Besthesda, MD, July 2003. 